CRYSTALLOGRAPHIC COMMUNICATIONS

ISSN 2056-9890

Received 5 November 2020

Accepted 20 November 2020

Edited by M. Weil, Vienna University of Technology, Austria

Keywords: crystal structure; thulium; nitrate; hydrate; hydrogen bonding.

CCDC references: 2045553; 2045552

Supporting information: this article has supporting information at journals.iucr.org/e

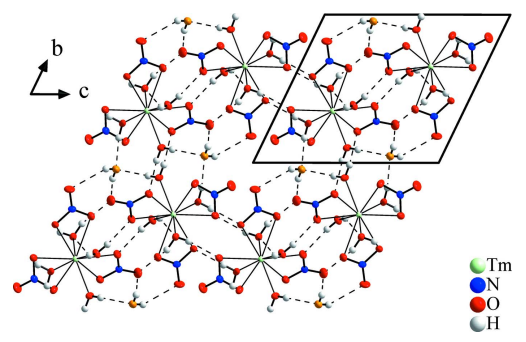

OPEN Ә ACCESS

\section{Crystal structures of the penta- and hexahydrate of thulium nitrate}

\author{
Wilhelm Klein*
}

Technische Universität München, Department of Chemistry, Lichtenbergstr. 4, 85747 Garching, Germany. *Correspondence e-mail: wilhelm.klein@tum.de

$\operatorname{Tm}\left(\mathrm{NO}_{3}\right)_{3} \cdot 5 \mathrm{H}_{2} \mathrm{O}$ and $\mathrm{Tm}\left(\mathrm{NO}_{3}\right)_{3} \cdot 6 \mathrm{H}_{2} \mathrm{O}$, or more precisely $\left[\mathrm{Tm}\left(\mathrm{NO}_{3}\right)_{3}\left(\mathrm{H}_{2} \mathrm{O}\right)_{4}\right] \cdot-$ $\mathrm{H}_{2} \mathrm{O}$ and $\left[\mathrm{Tm}\left(\mathrm{NO}_{3}\right)_{3}\left(\mathrm{H}_{2} \mathrm{O}\right)_{4}\right] \cdot 2 \mathrm{H}_{2} \mathrm{O}$, respectively, have been obtained from a concentrated solution of $\mathrm{Tm}_{2} \mathrm{O}_{3}$ in $\mathrm{HNO}_{3}$. The crystal structures of the two hydrates show strong similarities as both crystallize in space group $P \overline{1}$ with all atoms at general positions and contain neutral, molecular $\left[\operatorname{Tm}\left(\mathrm{NO}_{3}\right)_{3}\left(\mathrm{H}_{2} \mathrm{O}\right)_{4}\right]$ complexes, i.e. ten-coordinated $\mathrm{Tm}^{\mathrm{III}}$ cations with three nitrate anions as bidentate ligands and four coordinating water molecules, and one or two additional crystal water molecules, respectively. All building units are connected by medium-strong to weak $\mathrm{O}-\mathrm{H} \cdots \mathrm{O}$ hydrogen bonds. $\mathrm{Tm}\left(\mathrm{NO}_{3}\right)_{3} \cdot 6 \mathrm{H}_{2} \mathrm{O}$ represents the maximally hydrated thulium nitrate as well as the heaviest rare earth nitrate hexahydrate known to date.

\section{Chemical context}

The nitrates of the rare earth metals have long been used to separate and purify these elements. For example, when thulium was discovered (Cleve, 1897), fusion of nitrates was already used to separate the element from the erbiumcontaining earth, and a hydrate of thulium nitrate in substance was already described more than 100 years ago with four equivalents of water of crystallization and of highly hygroscopic nature (James, 1911). Later, among others, double nitrates like $\mathrm{Mg}_{3} L_{2}\left(\mathrm{NO}_{3}\right)_{12} \cdot 24 \mathrm{H}_{2} \mathrm{O}$ and $\left(\mathrm{NH}_{4}\right)_{2} \operatorname{Ln}\left(\mathrm{NO}_{3}\right)_{5} \cdot-$ $4 \mathrm{H}_{2} \mathrm{O}(\mathrm{Ln}=$ rare earth element $)$ were used to separate the elements by means of fractional crystallization (Prandtl, 1938). Also, when more sophisticated separation procedures such as chromatographic methods and solvent extraction were developed (Bock, 1950), there was still considerable interest in these complex nitrates because of their high solubility even in organic solvents. Numerous structural investigations have been reported for this family, not least for the hydrated compounds (Wickleder, 2002).

Considering the structural information for the maximally hydrated rare earth nitrates, a general tendency of a decreasing amount of water with increasing atomic number is obvious: for the lighter homologues $\mathrm{La}-\mathrm{Nd}$ and $\mathrm{Sm}-\mathrm{Tb}$, the hexahydrates are found as maximally hydrated compounds for the nitrates (La: Eriksson et al., 1980; Ce: Milinski et al., 1980; Pr: Decadt et al., 2012; Nd: Rogers et al., 1983; Sm: Kawashima et al., 2000; Eu: Stumpf \& Bolte, 2001; Gd: Taha et al., 2012; Tb: Moret et al., 1990) while for the heavier elements Dy-Er and $\mathrm{Yb}$, only pentahydrates have been reported (Ho: Rincke et al., 2017; other: Junk et al., 1999). Confirming this trend, the highest hydrate of Lu nitrate is the tetrahydrate (Junk et al., 1999), and for Tm the trihydrate exhibits the highest number of water molecules reported so far (Riess, 2012). 
In the present research communication, the new penta- and hexahydrates of $\operatorname{Tm}\left(\mathrm{NO}_{3}\right)_{3}$ are reported. While the pentahydrate of $\mathrm{Tm}\left(\mathrm{NO}_{3}\right)_{3}$ fills the gap within the known compounds containing $\mathrm{Er}$ and $\mathrm{Yb}$, the hexahydrate indeed represents the highest hydrated nitrate including $\mathrm{Tm}$ and shifts the border of known stable compounds notably to heavier rare earth elements.

\section{Structural commentary}

$\mathrm{Tm}\left(\mathrm{NO}_{3}\right)_{3} \cdot 5 \mathrm{H}_{2} \mathrm{O}$ crystallizes in the $\mathrm{Y}\left(\mathrm{NO}_{3}\right)_{3} \cdot 5 \mathrm{H}_{2} \mathrm{O}$ type of structure (Eriksson, 1982) in space group $P \overline{1}$ with all atoms at general positions. The structure consists of isolated molecular $\left[\mathrm{Tm}\left(\mathrm{NO}_{3}\right)_{3}\left(\mathrm{H}_{2} \mathrm{O}\right)_{4}\right]$ complexes and one additional free water molecule per formula unit (Fig. 1). The nitrate anions act as bidentate ligands so the $\mathrm{Tm}^{\mathrm{III}}$ atom is tenfold coordinated. The nitrate ions form an equatorial belt separating one aqua ligand from the other three, and are slightly inclined in the same sense and form a propeller-like shape. The nitrate anions coordinate asymmetrically at one shorter [2.3980(17)2.4479 (16) ^] and one slightly longer distance [2.5081 (16)$2.6193(18) \AA]$ each. The shortest Tm-O bonds [2.3235 (17)$2.3526(18) \AA]$ are formed with the three aqua ligands on the same side of the plane, while the remaining $\mathrm{Tm}-\mathrm{O}(\mathrm{H} 2)$ bond is in the range of the shorter bonds to the nitrate ions. The anions are almost planar with an $\mathrm{O}-\mathrm{N}-\mathrm{O}$ angular sum of $360.0^{\circ}$ where the angle formed by the coordinating $\mathrm{O}$ atoms is significantly reduced. The $\mathrm{N}-\mathrm{O}$ bond lengths are between 1.256 (3) and 1.290 (3) $\AA$ for coordinating and 1.213 (3) and 1.220 (3) $\AA$ for non-coordinating $\mathrm{O}$ atoms. Within the water molecules, the $\mathrm{O}-\mathrm{H}$ bond lengths are between 0.68 (6) and 0.86 (4) $\AA$, and the $\mathrm{H}-\mathrm{O}-\mathrm{H}$ angles between 102 (4) and $111(4)^{\circ}$. The structural entities, i.e. the molecular $\left[\mathrm{Tm}\left(\mathrm{NO}_{3}\right)_{3}\left(\mathrm{H}_{2} \mathrm{O}\right)_{4}\right]$ complexes and $\mathrm{H}_{2} \mathrm{O}$ molecules, are interconnected by almost linear hydrogen bonds (see Fig. 2) of medium-strong to weak strength. In detail, eight of ten independent $\mathrm{H}$ atoms form hydrogen bonds shorter than

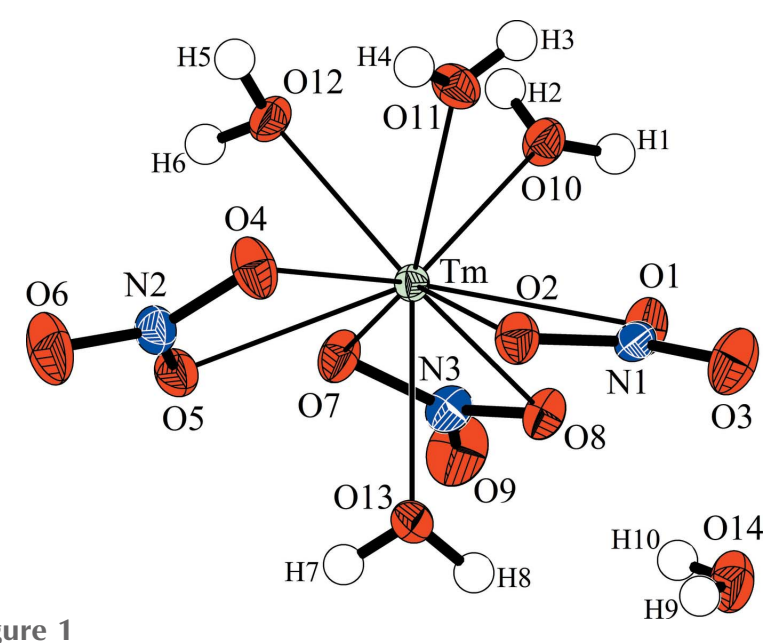

Figure 1

Asymmetric unit of $\operatorname{Tm}\left(\mathrm{NO}_{3}\right)_{3} \cdot 5 \mathrm{H}_{2} \mathrm{O}$ with the atom-numbering scheme. Anisotropic displacement ellipsoids for non-H atoms are drawn at the $50 \%$ probability level.
Table 1

Hydrogen-bond geometry $\left(\AA{ }^{\circ}\right)$ for $\operatorname{Tm}\left(\mathrm{NO}_{3}\right)_{3} \cdot 5 \mathrm{H}_{2} \mathrm{O}$.

\begin{tabular}{|c|c|c|c|c|}
\hline$D-\mathrm{H} \cdots A$ & $D-\mathrm{H}$ & $\mathrm{H} \cdots A$ & $D \cdots A$ & $D-\mathrm{H} \cdots A$ \\
\hline $\mathrm{O} 10-\mathrm{H} 1 \cdots \mathrm{O} 1^{\mathrm{i}}$ & $0.82(4)$ & $2.03(4)$ & $2.849(2)$ & $174(3)$ \\
\hline $\mathrm{O} 10-\mathrm{H} 2 \cdots \mathrm{O} 13^{\mathrm{ii}}$ & $0.78(4)$ & $2.32(4)$ & $2.996(3)$ & $145(3)$ \\
\hline $\mathrm{O} 10-\mathrm{H} 2 \cdots \mathrm{O} 2^{\mathrm{ii}}$ & $0.78(4)$ & $2.48(4)$ & $3.035(3)$ & $129(3)$ \\
\hline $\mathrm{O} 11-\mathrm{H} 3 \cdots \mathrm{O} 14^{\mathrm{i}}$ & $0.77(5)$ & $1.98(5)$ & $2.739(3)$ & $167(5)$ \\
\hline $\mathrm{O} 11-\mathrm{H} 4 \cdots \mathrm{O} 2^{\mathrm{iii}}$ & $0.85(4)$ & $2.03(4)$ & $2.874(2)$ & $171(4)$ \\
\hline $\mathrm{O} 12-\mathrm{H} 5 \cdots \mathrm{O} 14^{\mathrm{iv}}$ & $0.83(4)$ & $1.90(4)$ & $2.715(3)$ & $165(4)$ \\
\hline $\mathrm{O} 12-\mathrm{H}_{6} \cdots \mathrm{O}^{\mathrm{v}}$ & $0.83(4)$ & $1.95(4)$ & $2.776(2)$ & $174(4)$ \\
\hline $\mathrm{O} 13-\mathrm{H} 7 \ldots \mathrm{O}^{\mathrm{vi}}$ & $0.82(5)$ & $1.98(5)$ & $2.784(2)$ & $166(4)$ \\
\hline $\mathrm{O} 13-\mathrm{H} 8 \cdots \mathrm{O} 3^{\mathrm{vii}}$ & $0.86(4)$ & $2.12(4)$ & $2.953(3)$ & $163(4)$ \\
\hline $\mathrm{O} 14-\mathrm{H} 9 \cdots \mathrm{O}^{\mathrm{vii}}$ & $0.84(4)$ & $2.27(5)$ & $3.095(3)$ & $167(4)$ \\
\hline $\mathrm{O} 14-\mathrm{H} 10 \cdots \mathrm{O} 9$ & $0.68(6)$ & $2.44(6)$ & $3.040(3)$ & $148(5)$ \\
\hline $\mathrm{O} 14-\mathrm{H} 10 \cdots \mathrm{O}^{\mathrm{viii}}$ & $0.68(6)$ & $2.62(6)$ & $3.132(4)$ & $134(5)$ \\
\hline
\end{tabular}

Symmetry codes: (i) $-x,-y+1,-z+1$; (ii) $x-1, y, z$; (iii) $-x+1,-y,-z+1$; (iv) $x, y-1, z$; (v) $-x,-y+1,-z$; (vi) $-x+1,-y+1,-z$; (vii) $-x+1,-y+1,-z+1$; (viii) $x-1, y+1, z$.

$2.30 \AA$ with $\mathrm{O}-\mathrm{H} \cdots \mathrm{O}$ angles greater than $163^{\circ}$, while atoms $\mathrm{H} 2$ and $\mathrm{H} 10$ are part of bifurcated and slightly longer hydrogen bonds (Table 1).

$\mathrm{Tm}\left(\mathrm{NO}_{3}\right)_{3} \cdot 6 \mathrm{H}_{2} \mathrm{O}$ also crystallizes in space group $P \overline{1}$ without occupying special positions and is isotypic with the respective Pr compound (Decadt et al., 2012). In comparison with $\mathrm{Tm}\left(\mathrm{NO}_{3}\right)_{3} \cdot 5 \mathrm{H}_{2} \mathrm{O}$ the volume (as determined at $223 \mathrm{~K}$ ) increases by $34.4 \AA^{3}$ for the two additional $\mathrm{H}_{2} \mathrm{O}$ molecules per unit cell. Further structural similarities to the pentahydrate include the presence of molecular $\left[\mathrm{Tm}\left(\mathrm{NO}_{3}\right)_{3}\left(\mathrm{H}_{2} \mathrm{O}\right)_{4}\right]$ complexes and free water molecules, but here two per formula unit. The $\left[\operatorname{Tm}\left(\mathrm{NO}_{3}\right)_{3}\left(\mathrm{H}_{2} \mathrm{O}\right)_{4}\right]$ complexes of the penta- and hexahydrates differ slightly, since in the latter the four water molecules and the three nitrate ligands accumulate on opposite sides of the complex (Fig. 3). With the nitrate anions as more or less bidentate ligands, again ten atoms are found in the first coordination sphere of the $\mathrm{Tm}^{\mathrm{III}}$ atom in $\operatorname{Tm}\left(\mathrm{NO}_{3}\right)_{3} \cdot 6 \mathrm{H}_{2} \mathrm{O}$. The resulting polyhedron can be described as a strongly distorted bicapped square antiprism. The shortest Tm $-\mathrm{O}$ bonds are observed to the aqua ligands [2.2897 (18)2.3360 (16) A] . Similar to the pentahydrate, the nitrate anions

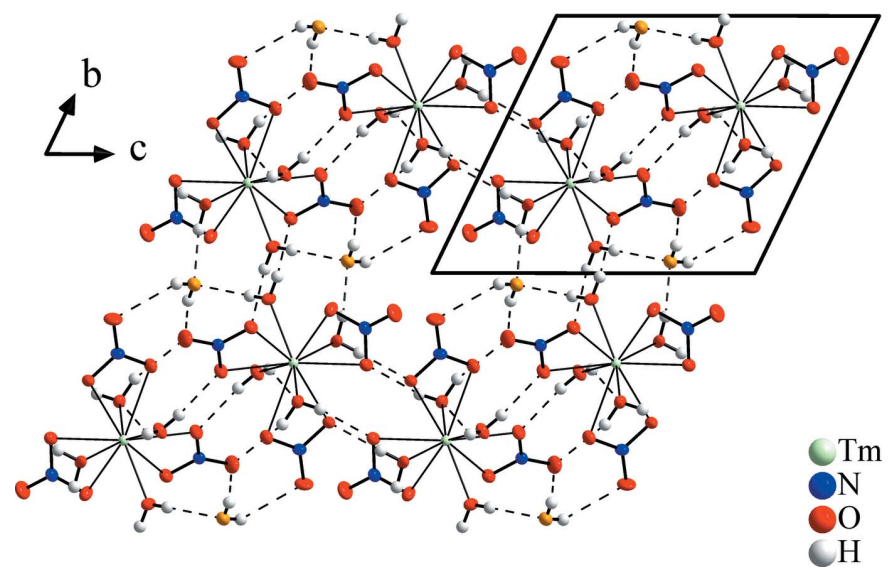

Figure 2

Crystal structure of $\mathrm{Tm}\left(\mathrm{NO}_{3}\right)_{3} \cdot 5 \mathrm{H}_{2} \mathrm{O}$ in a view along [100]. Hydrogen bonds are shown as dotted lines up to an $\mathrm{O} \cdots \mathrm{H}$ distance of $2.45 \AA$. Anisotropic displacement ellipsoids of non-H atoms are drawn at the $50 \%$ probability level. 


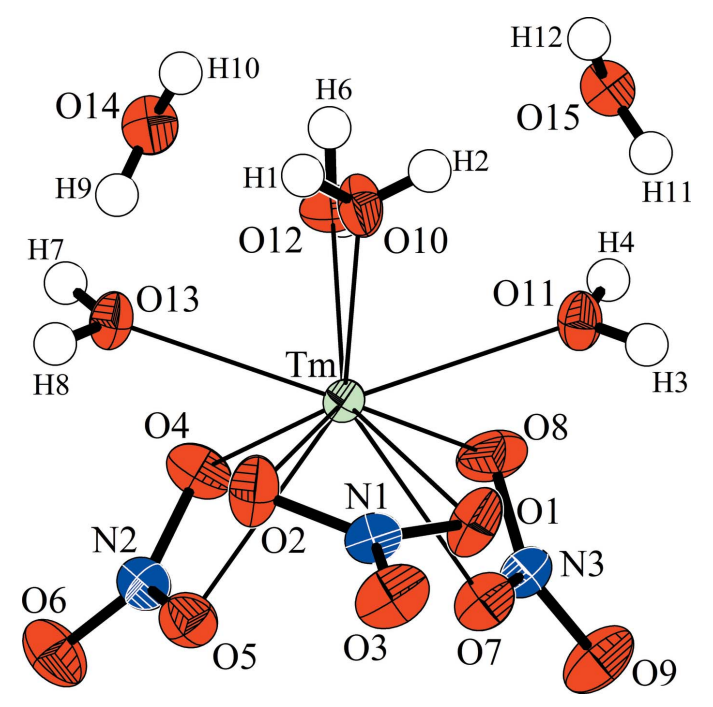

Figure 3

Asymmetric unit of $\operatorname{Tm}\left(\mathrm{NO}_{3}\right)_{3} \cdot 6 \mathrm{H}_{2} \mathrm{O}$ with the atom-numbering scheme. Anisotropic displacement ellipsoids for non- $\mathrm{H}$ atoms are drawn at the $50 \%$ probability level.

show an asymmetric coordination with one shorter [2.4039 (17)-2.4677 (17) $\AA$ ] and one longer Tm-O distance [2.5034 (18), 2.5252 (18), 2.991 (2) $\AA$ ] each. In one case, this is so severe that the corresponding $\mathrm{Tm}-\mathrm{O}$ distance is even larger than the distance between the $\mathrm{Tm}^{\mathrm{III}}$ atom and the central $\mathrm{N}$ atoms of the two remaining anions, and the arrangement should therefore rather be described as a $[9+1]$ coordination. The reason for this is probably the missing space in the coordination sphere of the $\mathrm{Tm}^{\mathrm{III}}$ atom, which makes such a distance increase necessary. A qualitatively analogous observation of a single extended $L n-\mathrm{N}$ distance was made for all isotypic compounds of other rare earth elements, but the relative extension of the distance in the structure described here is much larger than in all other examples, as can be expected for the representative with the smallest ion radius so far. The consideration including a reduced coordination number for the $\mathrm{Tm}^{\mathrm{III}}$ atom is supported by the different shape of the respective nitrate ion. While two anions are very similar

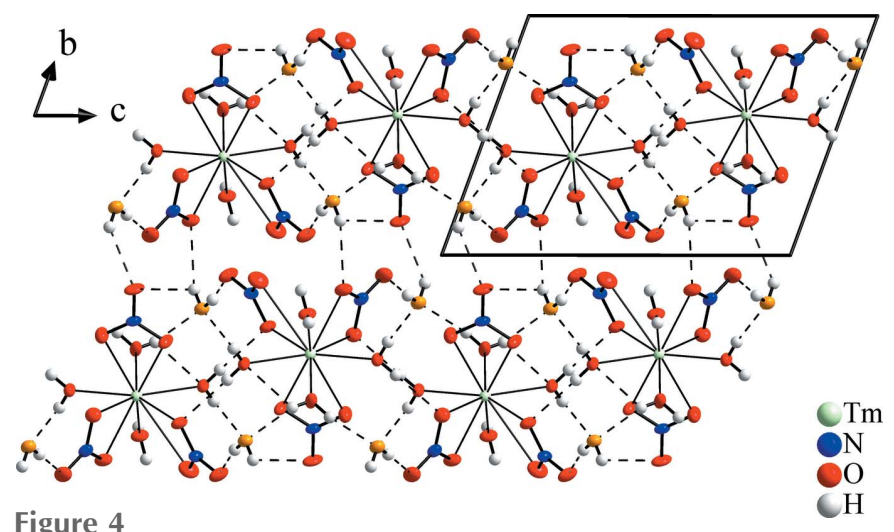

Figure 4

Crystal structure of $\operatorname{Tm}\left(\mathrm{NO}_{3}\right)_{3} \cdot 6 \mathrm{H}_{2} \mathrm{O}$ in a view along [100]. Hydrogen bonds are shown as dotted lines up to an $\mathrm{O} \cdots \mathrm{H}$ distance of $2.5 \AA$. Anisotropic displacement ellipsoids of non- $\mathrm{H}$ atoms are drawn at the $50 \%$ probability level.
Table 2

Hydrogen-bond geometry $\left(\AA{ }^{\circ}\right)$ for $\operatorname{Tm}\left(\mathrm{NO}_{3}\right)_{3} \cdot 6 \mathrm{H}_{2} \mathrm{O}$.

\begin{tabular}{|c|c|c|c|c|}
\hline$D-\mathrm{H} \cdots A$ & $D-\mathrm{H}$ & $\mathrm{H} \cdots A$ & $D \cdots A$ & $D-\mathrm{H} \cdots A$ \\
\hline $\mathrm{O} 10-\mathrm{H} 1 \cdots \mathrm{O} 14$ & $0.85(4)$ & $1.89(4)$ & $2.730(3)$ & $170(4)$ \\
\hline $\mathrm{O} 10-\mathrm{H} 2 \cdots \mathrm{O} 15$ & $0.73(5)$ & $2.00(5)$ & $2.714(3)$ & $164(5)$ \\
\hline $\mathrm{O} 11-\mathrm{H} 4 \cdots \mathrm{O} 15^{\mathrm{i}}$ & $0.74(4)$ & $1.93(4)$ & $2.666(2)$ & $174(4)$ \\
\hline $\mathrm{O} 11-\mathrm{H} 3 \cdots \mathrm{O} 7^{\mathrm{ii}}$ & $0.81(4)$ & $2.20(4)$ & $3.006(2)$ & $175(4)$ \\
\hline $\mathrm{O} 12-\mathrm{H} 5 \cdots \mathrm{O} 8^{\mathrm{iii}}$ & $0.80(4)$ & $2.15(4)$ & $2.943(3)$ & $172(4)$ \\
\hline $\mathrm{O} 12-\mathrm{H} 6 \cdots \mathrm{O}^{\mathrm{iv}}$ & $0.79(4)$ & $2.57(4)$ & $3.260(3)$ & $147(4)$ \\
\hline $\mathrm{O} 12-\mathrm{H} 6 \cdots \mathrm{O}^{\mathrm{iv}}$ & $0.79(4)$ & $2.61(4)$ & $3.269(3)$ & $142(4)$ \\
\hline $\mathrm{O} 13-\mathrm{H} 7 \cdots \mathrm{O} 14^{\mathrm{v}}$ & $0.78(4)$ & $1.93(4)$ & $2.713(3)$ & $174(4)$ \\
\hline $\mathrm{O} 13-\mathrm{H} 8 \cdots \mathrm{O}^{\mathrm{vi}}$ & $0.83(5)$ & $2.13(5)$ & $2.963(2)$ & $179(5)$ \\
\hline $\mathrm{O} 14-\mathrm{H} 9 \cdots \mathrm{O}^{\mathrm{vi}}$ & $0.80(4)$ & $2.01(4)$ & $2.804(3)$ & $176(4)$ \\
\hline $\mathrm{O} 14-\mathrm{H} 10 \cdots \mathrm{O}^{\mathrm{iv}}$ & $0.80(5)$ & $2.64(5)$ & $3.111(3)$ & $119(4)$ \\
\hline $\mathrm{O} 14-\mathrm{H} 10 \cdots \mathrm{O} 3^{\mathrm{vii}}$ & $0.80(5)$ & $2.24(5)$ & $2.885(2)$ & $138(4)$ \\
\hline $\mathrm{O} 15-\mathrm{H} 11 \cdots \mathrm{O} 9^{\mathrm{ii}}$ & $0.79(5)$ & $2.01(5)$ & $2.782(3)$ & $168(4)$ \\
\hline $\mathrm{O} 15-\mathrm{H} 12 \cdots \mathrm{O} 4^{\mathrm{viii}}$ & $0.79(5)$ & $2.29(5)$ & $2.926(2)$ & $137(4)$ \\
\hline $\mathrm{O} 15-\mathrm{H} 12 \cdots \mathrm{O}^{\mathrm{iv}}$ & $0.79(5)$ & $2.47(5)$ & $2.992(3)$ & $125(4)$ \\
\hline
\end{tabular}

Symmetry codes: (i) $-x+1,-y+1,-z+1$; (ii) $\quad-x,-y+1,-z+1$; (iii) $-x+1,-y,-z+1$; (iv) $x+1, y, z$; (v) $-x+1,-y+1,-z$; (vi) $-x,-y+1,-z$; (vii) $-x,-y+2,-z ;$ (viii) $x, y+1, z$.

with two longer and one shorter $\mathrm{N}-\mathrm{O}$ bonds for coordinating and non-coordinating $\mathrm{O}$ atoms, respectively, and one reduced $\mathrm{O}-\mathrm{N}-\mathrm{O}$ angle between the coordinating $\mathrm{O}$ atoms, the third anion exhibits only one longer $\mathrm{N}-\mathrm{O}$ bond of 1.275 (2) $\AA$, indicating the coordinating $\mathrm{O}$ atom, and two shorter and almost equal $\mathrm{N}-\mathrm{O}$ distances of 1.232 (3) $\AA$ and 1.236 (3) $\AA$ with more regular $\mathrm{O}-\mathrm{N}-\mathrm{O}$ angles. However, all nitrate ions are planar with an $\mathrm{O}-\mathrm{N}-\mathrm{O}$ angular sum of $360.0^{\circ}$. The water molecules show $\mathrm{O}-\mathrm{H}$ bond lengths between 0.73 (5)and 0.85 (4) $\AA$ and $\mathrm{H}-\mathrm{O}-\mathrm{H}$ angles between 105 (5) and 112 (4) ${ }^{\circ}$. The metal complexes and the water molecules build a threedimensional network of hydrogen bonds, again of mediumstrong to weak character (Table 2, Fig. 4). Nine of twelve independent $\mathrm{H}$ atoms form hydrogen bonds shorter than $2.2 \AA$ with $\mathrm{O}-\mathrm{H} \cdots \mathrm{O}$ angles greater than $164^{\circ}$ while $\mathrm{H} 6, \mathrm{H} 10$, and $\mathrm{H} 12$ are involved in weak and bifurcated hydrogen bonds.

Interestingly, the molecular Tm complexes in both crystal structures exhibit an alleged higher symmetry, viz. a threefold rotation axis in the pentahydrate and a mirror plane in the hexahydrate, as illustrated in Fig. 5. However, these are
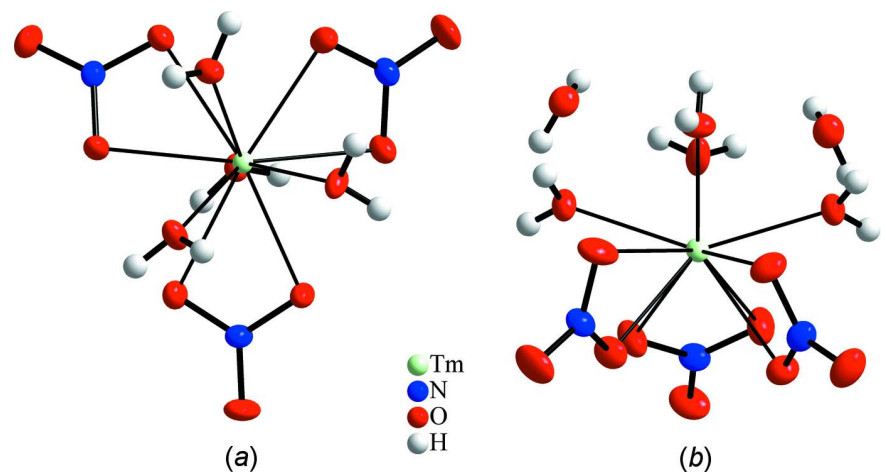

Figure 5

(b)

Structural details to emphasize the molecular pseudo-symmetry in the title compounds: $(a)$ a pseudo-threefold rotation axis in the molecular complex present in $\mathrm{Tm}\left(\mathrm{NO}_{3}\right)_{3} \cdot 5 \mathrm{H}_{2} \mathrm{O} ;(b)$ a pseudo-mirror plane in the molecular complex present in $\mathrm{Tm}\left(\mathrm{NO}_{3}\right)_{3} \cdot 6 \mathrm{H}_{2} \mathrm{O}$. Anisotropic displacement ellipsoids of non- $\mathrm{H}$ atoms are drawn at the $50 \%$ probability level. 
Table 3

Experimental details.

\begin{tabular}{|c|c|c|}
\hline & $\operatorname{Tm}\left(\mathrm{NO}_{3}\right)_{3} \cdot 5 \mathrm{H}_{2} \mathrm{O}$ & $\operatorname{Tm}\left(\mathrm{NO}_{3}\right)_{3} \cdot 6 \mathrm{H}_{2} \mathrm{O}$ \\
\hline Chemical formula & {$\left[\mathrm{Tm}\left(\mathrm{NO}_{3}\right)_{3}\left(\mathrm{H}_{2} \mathrm{O}\right)_{4}\right] \cdot \mathrm{H}_{2} \mathrm{O}$} & {$\left[\mathrm{Tm}\left(\mathrm{NO}_{3}\right)_{3}\left(\mathrm{H}_{2} \mathrm{O}\right)_{4}\right] \cdot 2 \mathrm{H}_{2} \mathrm{O}$} \\
\hline$M_{\mathrm{r}}$ & 445.04 & 463.06 \\
\hline Temperature (K) & 223 & 223 \\
\hline$a, b, c(\AA)$ & $6.5782(4), 9.5213(5), 10.4848(6)$ & $6.7050(3), 8.9733(4), 11.4915(6)$ \\
\hline$\alpha, \beta, \gamma\left({ }^{\circ}\right)$ & $63.696(4), 84.656(5), 76.146(4)$ & $70.924(4), 88.908(4), 68.923(4)$ \\
\hline$\mu\left(\mathrm{mm}^{-1}\right)$ & 7.85 & 7.41 \\
\hline Crystal size $(\mathrm{mm})$ & $0.25 \times 0.2 \times 0.2$ & $0.4 \times 0.2 \times 0.15$ \\
\hline \multicolumn{3}{|l|}{ Data collection } \\
\hline Diffractometer & Stoe StadiVari & Stoe StadiVari \\
\hline Absorption correction & $\begin{array}{l}\text { Empirical (using intensity measurements) } \\
\quad(X-A R E A ; \text { Stoe, } 2015)\end{array}$ & $\begin{array}{l}\text { Empirical (using intensity measurements) } \\
\quad(X-A R E A ; \text { Stoe, } 2015)\end{array}$ \\
\hline$(\sin \theta / \lambda)_{\max }\left(\AA^{-1}\right)$ & 0.756 & 0.756 \\
\hline \multicolumn{3}{|l|}{ Refinement } \\
\hline$R\left[F^{2}>2 \sigma\left(F^{2}\right)\right], w R\left(F^{2}\right), S$ & $0.017,0.029,0.65$ & $0.016,0.035,0.91$ \\
\hline No. of reflections & 4113 & 4385 \\
\hline No. of parameters & 204 & 221 \\
\hline $\mathrm{H}$-atom treatment & All H-atom parameters refined & All H-atom parameters refined \\
\hline$\Delta \rho_{\max }, \Delta \rho_{\min }\left(\mathrm{e} \AA^{-3}\right)$ & $0.94,-0.92$ & $1.11,-1.19$ \\
\hline
\end{tabular}

Computer programs: X-AREA (Stoe, 2015), SHELXS97 (Sheldrick, 2008), SHELXL2014/7 (Sheldrick, 2015), DIAMOND (Brandenburg \& Putz, 2012) and publCIF (Westrip, 2010).

pseudo-symmetries, with the higher symmetry violated at a molecular level and in the first coordination sphere, and incompatible with the space-group symmetry.

\section{Database survey}

The crystal structure of anhydrous $\mathrm{Tm}\left(\mathrm{NO}_{3}\right)_{3}$ was determined quite recently (Heinrichs, 2013), and one hydrated phase has been reported so far, i.e. the trihydrate (Riess, 2012). In addition, basic oxo-hydroxo-nitrate hydrates are known with Tm (Giester et al., 2009). The thulium nitrate pentahydrate adopts the $\mathrm{Y}\left(\mathrm{NO}_{3}\right)_{3} \cdot 5 \mathrm{H}_{2} \mathrm{O}$ type of structure (Eriksson, 1982; Klein, 2020), and is isotypic with the respective Eu (Ribár et al., 1986), Gd (Stockhause \& Meyer, 1997), Dy, Er, Yb (Junk et al., 1999) and Ho compounds (Rincke et al., 2017). $\mathrm{Tm}\left(\mathrm{NO}_{3}\right)_{3} \cdot 6 \mathrm{H}_{2} \mathrm{O}$ is isotypic with the nitrate hexahydrates of $\mathrm{Y}$ (Ribár et al., 1980), Pr (Rumanova et al., 1964; Fuller \& Jacobsen, 1976; Decadt et al., 2012), Nd (Rogers et al., 1983; Shi \& Wang, 1991), Sm (Shi \& Wang, 1990; Kawashima et al., 2000), Eu (Stumpf \& Bolte, 2001; Ananyev et al., 2016), Gd (Ma et al., 1991; Taha et al., 2012) and Tb (Moret et al., 1990).

\section{Synthesis and crystallization}

$\left[\mathrm{Tm}\left(\mathrm{NO}_{3}\right)_{3}\left(\mathrm{H}_{2} \mathrm{O}\right)_{4}\right] \cdot \mathrm{H}_{2} \mathrm{O}$ was prepared by dissolving $\mathrm{Tm}_{2} \mathrm{O}_{3}$ (Fluka AG; $99.9 \%$ ) in hot aqueous nitric acid $\left(65 \%_{\mathrm{wt}}\right)$. From saturated solutions, crystals with sizes up to the millimetre range were grown at room temperature within one day. Single crystals were removed, cleansed from the mother liquor and placed on a microscope slide in air. For the single-crystal data collection, crystals were immersed into perfluoroalkyl ether, which also acts as glue on a glass tip during the measurement. The remaining crystals were carefully ground to measure an $\mathrm{X}$-ray powder pattern that, according to a comparison with the pattern simulated from the single-crystal structure determination, showed exclusively reflections of the pentahydrate. The crystals are hygroscopic and usually deliquesce within hours under ambient conditions depending on air humidity. Rapid re-crystallization within minutes can be induced by scratching on the glass slide. Surprisingly, from one recrystallization the hexahydrate $\left[\mathrm{Tm}\left(\mathrm{NO}_{3}\right)_{3}\left(\mathrm{H}_{2} \mathrm{O}\right)_{4}\right] \cdot 2 \mathrm{H}_{2} \mathrm{O}$ was obtained. All investigated crystals from this batch revealed the unit cell of the hexahydrate, so the crystallization seemed to result in a pure product in this case as well. Optically indistinguishable, the crystals of the hexahydrate showed the same deliquescence behaviour. It has not been possible to determine the exact conditions required to obtain the hexahydrate so far. According to EDX measurements, the crystals contain $\mathrm{Tm}$ as the only element heavier than oxygen.

\section{Refinement}

Crystal data, data collection, and structure refinement details are summarized in Table 3. In both structure refinements, all hydrogen atoms have been located from difference Fourier 
maps and refined with free atomic coordinates and isotropic displacement parameters.

\section{References}

Ananyev, I. V., Nelyubina, Yu. V., Puntus, L. N., Lyssenko, K. A. \& Eremenko, I. L. (2016). Russ. Chem. Bull. 65, 1178-1188.

Bock, R. (1950). Angew. Chem. 62, 375-382.

Brandenburg, K. \& Putz, H. (2012). DIAMOND. Crystal Impact GbR, Bonn, Germany.

Cleve, P. T. (1897). C. R. Hebd. Seances Acad. Sci. 89, 328-329.

Decadt, R., Van Der Voort, P., Van Driessche, I., Van Deun, R. \& Van Hecke, K. (2012). Acta Cryst. E68, i59-i60.

Eriksson, B. (1982). Acta Chem. Scand. A36, 186-188.

Eriksson, B., Larsson, L. O., Niinistö, L. \& Valkonen, J. (1980). Inorg. Chem. 19, 1207-1210.

Fuller, C. C. \& Jacobsen, R. A. (1976). Cryst. Struct. Commun. 5, 349352.

Giester, G., Žák, Z. \& Unfried, P. (2009). J. Alloys Compd. 481, 116128.

Heinrichs, C. (2013). Dissertation, Universität zu Köln, Köln, Germany.

James, C. (1911). J. Am. Chem. Soc. 33, 1332-1344.

Junk, P. C., Kepert, D. L., Skelton, B. W. \& White, A. H. (1999). Aust. J. Chem. 52, 497-505.

Kawashima, R., Sasaki, M., Satoh, S., Isoda, H., Kino, Y. \& Shiozaki, Y. (2000). J. Phys. Soc. Jpn, 69, 3297-3303.

Klein, W. (2020). Z. Kristallogr. New Cryst. Struct. 235, 801-802.

Ma, H., Gao, S. \& Yang, Z. (1991). Wuji Huaxue Xиebao, 7, 351-353.
Milinski, N., Ribár, B. \& Satarić, M. (1980). Cryst. Struct. Commun. 9 , 473-476.

Moret, E., Bünzli, J. G. \& Schenk, K. J. (1990). Inorg. Chim. Acta, 178, 83-88.

Prandtl, W. (1938). Z. Anorg. Allg. Chem. 238, 321-334.

Ribár, B., Kapor, A., Argay, Gy. \& Kálmán, A. (1986). Acta Cryst. C42, 1450-1452.

Ribár, B., Milinski, N., Budovalcev, Z. \& Krstanović, I. (1980). Cryst. Struct. Commun. 9, 203-206.

Riess, K. (2012). Dissertation. Carl von Ossietzky Universität, Oldenburg, Germany.

Rincke, C., Schmidt, H. \& Voigt, W. (2017). Z. Anorg. Allg. Chem. 643, 437-442.

Rogers, D. J., Taylor, N. J. \& Toogood, G. E. (1983). Acta Cryst. C39, 939-941.

Rumanova, I. M., Volodina, G. F. \& Belov, N. V. (1964). Kristallografiya, 9, 642-654.

Sheldrick, G. M. (2008). Acta Cryst. A64, 112-122.

Sheldrick, G. M. (2015). Acta Cryst. C71, 3-8.

Shi, B. D. \& Wang, J. Z. (1990). Jiegou Huaxue, 9, 164-167.

Shi, B. D. \& Wang, J. Z. (1991). Xiamen Daxue Xuebao Ziran Kexueban 30, 55-58.

Stockhause, S. \& Meyer, G. (1997). Z. Kristallogr. New Cryst. Struct. 212, 315.

Stoe (2015). $X$-AREA. Stoe \& Cie, Darmstadt, Germany.

Stumpf, T. \& Bolte, M. (2001). Acta Cryst. E57, i10-i11.

Taha, Z. A., Ajlouni, A., Hijazi, A. K., Kühn, F. E. \& Herdtweck, E. (2012). Acta Cryst. E68, i56-i57.

Westrip, S. P. (2010). J. Appl. Cryst. 43, 920-925.

Wickleder, M. S. (2002). Chem. Rev. 102, 2011-2088. 


\section{supporting information}

Acta Cryst. (2020). E76, 1863-1867 [https://doi.org/10.1107/S2056989020015388]

\section{Crystal structures of the penta- and hexahydrate of thulium nitrate}

\section{Wilhelm Klein}

\section{Computing details}

For both structures, data collection: $X$-AREA (Stoe, 2015); cell refinement: $X$-AREA (Stoe, 2015); data reduction: $X$ AREA (Stoe, 2015); program(s) used to solve structure: SHELXS97 (Sheldrick, 2008); program(s) used to refine structure: SHELXL2014/7 (Sheldrick, 2015); molecular graphics: DIAMOND (Brandenburg \& Putz, 2012); software used to prepare material for publication: publCIF (Westrip, 2010).

Thulium nitrate (TONi-2_223K)

Crystal data

$\left[\mathrm{Tm}\left(\mathrm{NO}_{3}\right)_{3}\left(\mathrm{H}_{2} \mathrm{O}\right)_{4}\right] \cdot \mathrm{H}_{2} \mathrm{O}$

$M_{r}=445.04$

Triclinic, $P \overline{1}$

$a=6.5782(4) \AA$

$b=9.5213(5) \AA$

$c=10.4848(6) \AA$

$\alpha=63.696(4)^{\circ}$

$\beta=84.656(5)^{\circ}$

$\gamma=76.146(4)^{\circ}$

$V=571.51(6) \AA^{3}$

\section{Data collection}

STOE StadiVari

\section{diffractometer}

Radiation source: Genix 3D HF Mo

Graded multilayer mirror monochromator

Detector resolution: 5.81 pixels $\mathrm{mm}^{-1}$

$\omega$ scans

Absorption correction: empirical (using intensity measurements)

(X-AREA; Stoe, 2015)

Refinement

Refinement on $F^{2}$

Least-squares matrix: full

$R\left[F^{2}>2 \sigma\left(F^{2}\right)\right]=0.017$

$w R\left(F^{2}\right)=0.029$

$S=0.65$

4113 reflections

204 parameters

0 restraints

Primary atom site location: structure-invariant direct methods
$Z=2$

$F(000)=424$

$D_{\mathrm{x}}=2.586 \mathrm{Mg} \mathrm{m}^{-3}$

Mo $K \alpha$ radiation, $\lambda=0.71073 \AA$

Cell parameters from 15951 reflections

$\theta=3.2-36.9^{\circ}$

$\mu=7.85 \mathrm{~mm}^{-1}$

$T=223 \mathrm{~K}$

Block, colourless

$0.25 \times 0.2 \times 0.2 \mathrm{~mm}$

$T_{\min }=0.798, T_{\max }=1.000$

18549 measured reflections

4113 independent reflections

3394 reflections with $I>2 \sigma(I)$

$R_{\text {int }}=0.038$

$\theta_{\max }=32.5^{\circ}, \theta_{\min }=3.2^{\circ}$

$h=-9 \rightarrow 9$

$k=-13 \rightarrow 14$

$l=-14 \rightarrow 15$

Secondary atom site location: difference Fourier map

Hydrogen site location: difference Fourier map

All $\mathrm{H}$-atom parameters refined

$w=1 /\left[\sigma^{2}\left(F_{\mathrm{o}}^{2}\right)\right]$

where $P=\left(F_{\mathrm{o}}^{2}+2 F_{\mathrm{c}}^{2}\right) / 3$

$(\Delta / \sigma)_{\max }=0.001$

$\Delta \rho_{\max }=0.94 \mathrm{e} \AA^{-3}$

$\Delta \rho_{\min }=-0.92$ e $\AA^{-3}$ 
Extinction correction: SHELXL-2014/7

(Sheldrick, 2015),

$\mathrm{Fc}^{*}=\mathrm{kFc}\left[1+0.001 \times \mathrm{Fc}^{2} \lambda^{3} / \sin (2 \theta)\right]^{-1 / 4}$

Extinction coefficient: $0.0130(4)$

\section{Special details}

Geometry. All esds (except the esd in the dihedral angle between two 1.s. planes) are estimated using the full covariance matrix. The cell esds are taken into account individually in the estimation of esds in distances, angles and torsion angles; correlations between esds in cell parameters are only used when they are defined by crystal symmetry. An approximate (isotropic) treatment of cell esds is used for estimating esds involving l.s. planes.

Fractional atomic coordinates and isotropic or equivalent isotropic displacement parameters $\left(\AA^{2}\right)$

\begin{tabular}{lllll}
\hline & $x$ & $y$ & $z$ & $U_{\text {iso }} * / U_{\text {eq }}$ \\
\hline Tm & $0.24261(2)$ & $0.34935(2)$ & $0.29463(2)$ & $0.01335(4)$ \\
N1 & $0.4678(3)$ & $0.2804(2)$ & $0.5557(2)$ & $0.0181(4)$ \\
O1 & $0.2946(3)$ & $0.38136(19)$ & $0.50762(18)$ & $0.0237(3)$ \\
O2 & $0.5532(3)$ & $0.21177(19)$ & $0.47971(17)$ & $0.0235(3)$ \\
O3 & $0.5463(4)$ & $0.2559(3)$ & $0.6667(2)$ & $0.0334(5)$ \\
N2 & $0.5187(3)$ & $0.2148(2)$ & $0.1245(2)$ & $0.0224(4)$ \\
O4 & $0.4995(3)$ & $0.1419(2)$ & $0.25849(18)$ & $0.0281(4)$ \\
O5 & $0.4049(3)$ & $0.35678(19)$ & $0.06621(17)$ & $0.0235(3)$ \\
O6 & $0.6366(4)$ & $0.1552(2)$ & $0.0567(2)$ & $0.0393(5)$ \\
N3 & $0.0240(3)$ & $0.6856(2)$ & $0.1466(2)$ & $0.0207(4)$ \\
O7 & $0.0612(3)$ & $0.58072(19)$ & $0.09609(18)$ & $0.0229(4)$ \\
O8 & $0.1039(3)$ & $0.63874(19)$ & $0.26683(17)$ & $0.0232(3)$ \\
O9 & $-0.0817(4)$ & $0.8199(2)$ & $0.0805(2)$ & $0.0371(5)$ \\
O10 & $-0.0906(3)$ & $0.3864(2)$ & $0.39356(19)$ & $0.0222(3)$ \\
H1 & $-0.150(6)$ & $0.447(4)$ & $0.428(4)$ & $0.034(8)^{*}$ \\
H2 & $-0.182(6)$ & $0.374(4)$ & $0.360(4)$ & $0.028(8)^{*}$ \\
O11 & $0.1965(3)$ & $0.1013(2)$ & $0.46732(19)$ & $0.0221(3)$ \\
H3 & $0.124(7)$ & $0.088(5)$ & $0.532(5)$ & $0.047(11)^{*}$ \\
H4 & $0.278(6)$ & $0.014(5)$ & $0.475(4)$ & $0.035(9)^{*}$ \\
O12 & $0.0243(3)$ & $0.2649(2)$ & $0.19433(18)$ & $0.0229(3)$ \\
H5 & $0.038(6)$ & $0.169(5)$ & $0.212(4)$ & $0.041(10)^{*}$ \\
H6 & $0.009(6)$ & $0.311(4)$ & $0.106(4)$ & $0.035(9)^{*}$ \\
O13 & $0.5125(3)$ & $0.5005(2)$ & $0.22335(18)$ & $0.0207(3)$ \\
H7 & $0.556(8)$ & $0.530(5)$ & $0.141(5)$ & $0.059(12)^{*}$ \\
H8 & $0.495(7)$ & $0.586(5)$ & $0.237(4)$ & $0.045(10)^{*}$ \\
O14 & $0.0035(5)$ & $0.9548(2)$ & $0.2793(2)$ & $0.0322(5)$ \\
H9 & $0.120(7)$ & $0.892(5)$ & $0.285(4)$ & $0.038(9)^{*}$ \\
H10 & $-0.046(9)$ & $0.956(6)$ & $0.224(6)$ & $0.066(16)^{*}$ \\
& & & &
\end{tabular}

Atomic displacement parameters $\left(\AA^{2}\right)$

\begin{tabular}{lllllll}
\hline & $U^{11}$ & $U^{22}$ & $U^{33}$ & $U^{12}$ & $U^{13}$ & $U^{23}$ \\
\hline Tm & $0.01449(5)$ & $0.01318(5)$ & $0.01242(4)$ & $-0.00290(3)$ & $-0.00004(3)$ & $-0.00565(3)$ \\
N1 & $0.0201(10)$ & $0.0177(8)$ & $0.0181(8)$ & $-0.0059(7)$ & $-0.0007(7)$ & $-0.0082(7)$ \\
O1 & $0.0221(9)$ & $0.0251(8)$ & $0.0241(8)$ & $0.0038(7)$ & $-0.0053(6)$ & $-0.0142(7)$
\end{tabular}




$\begin{array}{lllllll}\text { O2 } & 0.0262(9) & 0.0215(7) & 0.0233(8) & -0.0017(7) & 0.0005(7) & -0.0118(6) \\ \text { O3 } & 0.0384(12) & 0.0372(10) & 0.0277(10) & -0.0024(9) & -0.0141(9) & -0.0170(8) \\ \text { N2 } & 0.0248(11) & 0.0220(9) & 0.0214(9) & -0.0042(8) & 0.0040(8) & -0.0117(8) \\ \text { O4 } & 0.0330(10) & 0.0251(8) & 0.0171(8) & 0.0029(7) & 0.0007(7) & -0.0059(6) \\ \text { O5 } & 0.0291(10) & 0.0197(7) & 0.0214(8) & -0.0053(7) & 0.0058(7) & -0.0097(6) \\ \text { O6 } & 0.0454(13) & 0.0381(10) & 0.0335(10) & 0.0016(9) & 0.0120(9) & -0.0224(8) \\ \text { N3 } & 0.0231(10) & 0.0150(8) & 0.0220(9) & -0.0010(7) & -0.0039(7) & -0.0072(7) \\ \text { O7 } & 0.0329(10) & 0.0166(7) & 0.0192(8) & -0.0016(7) & -0.0056(7) & -0.0084(6) \\ \text { O8 } & 0.0266(9) & 0.0240(8) & 0.0225(8) & -0.0042(7) & -0.0037(7) & -0.0131(6) \\ \text { O9 } & 0.0451(13) & 0.0182(8) & 0.0380(10) & 0.0105(8) & -0.0137(9) & -0.0093(8) \\ \text { O10 } & 0.0165(9) & 0.0288(8) & 0.0288(9) & -0.0047(7) & 0.0026(7) & -0.0198(7) \\ \text { O11 } & 0.0238(9) & 0.0172(7) & 0.0205(8) & -0.0036(7) & 0.0038(7) & -0.0051(6) \\ \text { O12 } & 0.0371(10) & 0.0185(8) & 0.0152(8) & -0.0110(7) & -0.0035(7) & -0.0062(6) \\ \text { O13 } & 0.0257(9) & 0.0196(7) & 0.0170(8) & -0.0078(6) & 0.0028(6) & -0.0073(6) \\ \text { O14 } & 0.0520(15) & 0.0212(9) & 0.0236(10) & -0.0045(9) & 0.0004(9) & -0.0117(8)\end{array}$

Geometric parameters $\left(\AA,{ }^{\circ}\right)$

\begin{tabular}{|c|c|c|c|}
\hline $\mathrm{Tm}-\mathrm{O} 12$ & $2.3235(18)$ & N3-O9 & $1.216(3)$ \\
\hline $\mathrm{Tm}-\mathrm{O} 11$ & $2.3235(17)$ & $\mathrm{N} 3-\mathrm{O} 8$ & $1.256(3)$ \\
\hline $\mathrm{Tm}-\mathrm{O} 10$ & $2.3526(18)$ & $\mathrm{N} 3-\mathrm{O} 7$ & $1.290(2)$ \\
\hline $\mathrm{Tm}-\mathrm{O} 7$ & $2.3980(17)$ & $\mathrm{O} 7-\mathrm{O} 8$ & $2.153(2)$ \\
\hline $\mathrm{Tm}-\mathrm{O} 13$ & $2.4089(18)$ & O7-O9 & $2.184(2)$ \\
\hline $\mathrm{Tm}-\mathrm{O} 4$ & $2.4181(17)$ & $\mathrm{O} 7-\mathrm{O} 12^{\mathrm{vii}}$ & $2.776(2)$ \\
\hline $\mathrm{Tm}-\mathrm{O} 1$ & $2.4479(16)$ & $\mathrm{O} 7-\mathrm{O} 12$ & $2.778(2)$ \\
\hline $\mathrm{Tm}-\mathrm{O} 5$ & $2.5081(16)$ & $\mathrm{O} 7-\mathrm{O} 10$ & $3.056(3)$ \\
\hline $\mathrm{Tm}-\mathrm{O} 8$ & $2.5776(15)$ & $\mathrm{O} 7-\mathrm{O} 13$ & $3.150(3)$ \\
\hline $\mathrm{Tm}-\mathrm{O} 2$ & $2.6193(18)$ & $\mathrm{O} 8-\mathrm{O} 9$ & $2.175(2)$ \\
\hline $\mathrm{Tm}-\mathrm{N} 2$ & 2.9035 (19) & $\mathrm{O} 8-\mathrm{O} 10$ & $2.738(3)$ \\
\hline $\mathrm{Tm}-\mathrm{N} 3$ & $2.9212(18)$ & $\mathrm{O} 8-\mathrm{O} 13$ & $2.788(2)$ \\
\hline $\mathrm{Tm}-\mathrm{N} 1$ & $2.9690(19)$ & $\mathrm{O} 8-\mathrm{O}^{\mathrm{iv}}$ & $2.969(3)$ \\
\hline $\mathrm{N} 1-\mathrm{O} 3$ & $1.220(3)$ & $\mathrm{O} 8-\mathrm{O} 14$ & $2.982(3)$ \\
\hline $\mathrm{N} 1-\mathrm{O} 2$ & $1.257(2)$ & $\mathrm{O} 9-\mathrm{O} 14$ & $3.040(3)$ \\
\hline $\mathrm{N} 1-\mathrm{O} 1$ & $1.276(3)$ & $\mathrm{O} 9-\mathrm{O}^{\mathrm{v}}$ & $3.153(3)$ \\
\hline $\mathrm{O} 1-\mathrm{O} 2$ & $2.144(2)$ & $\mathrm{O} 9-\mathrm{O}^{\mathrm{viii}}$ & 3.199 (2) \\
\hline $\mathrm{O} 1-\mathrm{O} 3$ & $2.180(3)$ & $\mathrm{O} 10-\mathrm{O} 11$ & $2.736(2)$ \\
\hline $\mathrm{O} 1-\mathrm{O} 8$ & $2.755(2)$ & $\mathrm{O} 10-\mathrm{O} 12$ & $2.775(2)$ \\
\hline $\mathrm{O} 1-\mathrm{O} 10^{\mathrm{i}}$ & 2.849 (2) & $\mathrm{O} 10-\mathrm{O} 1^{\mathrm{i}}$ & $2.849(2)$ \\
\hline $\mathrm{O} 1-\mathrm{O} 10$ & $2.882(3)$ & $\mathrm{O} 10-\mathrm{O} 13^{\mathrm{ix}}$ & $2.996(3)$ \\
\hline $\mathrm{O} 1-\mathrm{O} 13$ & $3.035(2)$ & $\mathrm{O} 10-\mathrm{O} 2^{\mathrm{ix}}$ & $3.035(3)$ \\
\hline $\mathrm{O} 1-\mathrm{O} 11$ & 3.095 (2) & $\mathrm{O} 10-\mathrm{H} 1$ & $0.82(4)$ \\
\hline $\mathrm{O} 2-\mathrm{O} 3$ & $2.174(2)$ & $\mathrm{O} 10-\mathrm{H} 2$ & $0.78(4)$ \\
\hline $\mathrm{O} 2-\mathrm{O} 4$ & $2.753(2)$ & $\mathrm{O} 11-\mathrm{O} 14^{\mathrm{i}}$ & $2.739(3)$ \\
\hline $\mathrm{O} 2-\mathrm{O} 11$ & $2.827(3)$ & $\mathrm{O} 11-\mathrm{O} 12$ & $2.777(2)$ \\
\hline $\mathrm{O} 2-\mathrm{O} 13$ & $2.844(2)$ & $\mathrm{O} 11-\mathrm{O} 2^{\mathrm{ii}}$ & $2.874(2)$ \\
\hline $\mathrm{O} 2-\mathrm{O} 11^{\mathrm{ii}}$ & $2.874(2)$ & $\mathrm{O} 11-\mathrm{O} 4^{\mathrm{ii}}$ & $3.253(2)$ \\
\hline $\mathrm{O} 2-\mathrm{O} 10^{\mathrm{iii}}$ & $3.035(3)$ & $\mathrm{O} 11-\mathrm{H} 3$ & $0.77(5)$ \\
\hline $\mathrm{O} 3-\mathrm{O} 13^{\mathrm{iv}}$ & $2.953(3)$ & $\mathrm{O} 11-\mathrm{H} 4$ & $0.85(4)$ \\
\hline
\end{tabular}




\begin{tabular}{|c|c|c|c|}
\hline $\mathrm{O} 3-\mathrm{O} 8^{\mathrm{iv}}$ & $2.969(3)$ & $012-014^{x}$ & $2.715(3)$ \\
\hline $\mathrm{O} 3-\mathrm{O} 14^{\mathrm{iv}}$ & $3.095(3)$ & $\mathrm{O} 12-\mathrm{O} 7^{\mathrm{vii}}$ & $2.776(2)$ \\
\hline $\mathrm{N} 2-\mathrm{O} 6$ & $1.213(3)$ & $\mathrm{O} 12-\mathrm{O} 9^{\mathrm{vii}}$ & $3.288(3)$ \\
\hline $\mathrm{N} 2-\mathrm{O} 4$ & $1.271(3)$ & $\mathrm{O} 12-\mathrm{H} 5$ & $0.83(4)$ \\
\hline $\mathrm{N} 2-\mathrm{O} 5$ & $1.277(3)$ & $\mathrm{O} 12-\mathrm{H} 6$ & $0.83(4)$ \\
\hline $\mathrm{O} 4-\mathrm{O} 5$ & $2.146(2)$ & $\mathrm{O} 13-\mathrm{O}^{\mathrm{v}}$ & $2.784(2)$ \\
\hline $\mathrm{O} 4-\mathrm{O} 6$ & $2.184(2)$ & $\mathrm{O} 13-\mathrm{O}^{\mathrm{iv}}$ & $2.953(3)$ \\
\hline $\mathrm{O} 4-\mathrm{O} 11$ & $2.779(3)$ & $\mathrm{O} 13-\mathrm{O} 10^{\mathrm{iii}}$ & $2.996(3)$ \\
\hline $\mathrm{O} 4-\mathrm{O} 12$ & $3.088(3)$ & $\mathrm{O} 13-\mathrm{O}^{\mathrm{v}}$ & $3.286(3)$ \\
\hline $\mathrm{O} 5-\mathrm{O} 6$ & $2.181(2)$ & $\mathrm{O} 13-\mathrm{H} 7$ & $0.82(5)$ \\
\hline $\mathrm{O} 5-\mathrm{O} 13^{\mathrm{v}}$ & $2.784(2)$ & $\mathrm{O} 13-\mathrm{H} 8$ & $0.86(4)$ \\
\hline $\mathrm{O} 5-\mathrm{O} 13$ & $2.786(2)$ & $\mathrm{O} 14-\mathrm{O} 12^{\mathrm{xi}}$ & $2.715(3)$ \\
\hline $\mathrm{O} 5-\mathrm{O} 7$ & $2.812(2)$ & $\mathrm{O} 14-\mathrm{O} 11^{\mathrm{i}}$ & $2.739(3)$ \\
\hline $\mathrm{O} 5-\mathrm{O} 12$ & $2.867(3)$ & $\mathrm{O} 14-\mathrm{O} 3^{\text {iv }}$ & 3.095 (3) \\
\hline $\mathrm{O} 5-\mathrm{O}^{\mathrm{v}}$ & $2.981(3)$ & $\mathrm{O} 14-\mathrm{O6}^{\mathrm{viii}}$ & $3.132(4)$ \\
\hline $\mathrm{O} 6-\mathrm{O} 14^{\mathrm{vi}}$ & $3.132(4)$ & $\mathrm{O} 14-\mathrm{H} 9$ & $0.84(4)$ \\
\hline $\mathrm{O} 6-\mathrm{O} 9^{\mathrm{v}}$ & $3.153(3)$ & $\mathrm{O} 14-\mathrm{H} 10$ & $0.68(6)$ \\
\hline $\mathrm{O} 6-\mathrm{O} 9^{\mathrm{vi}}$ & $3.199(2)$ & & \\
\hline $\mathrm{O} 12-\mathrm{Tm}-\mathrm{O} 11$ & $73.38(6)$ & $\mathrm{N} 3-\mathrm{O} 8-\mathrm{O} 1$ & $146.83(13)$ \\
\hline $\mathrm{O} 12-\mathrm{Tm}-\mathrm{O} 10$ & $72.80(7)$ & $\mathrm{O} 7-\mathrm{O} 8-\mathrm{O} 1$ & $114.60(8)$ \\
\hline $\mathrm{O} 11-\mathrm{Tm}-\mathrm{O} 10$ & $71.63(6)$ & $\mathrm{O} 9-\mathrm{O} 8-\mathrm{O} 1$ & $171.15(11)$ \\
\hline $\mathrm{O} 12-\mathrm{Tm}-\mathrm{O} 7$ & 72.07 (6) & $\mathrm{Tm}-\mathrm{O} 8-\mathrm{O} 1$ & $54.53(5)$ \\
\hline $\mathrm{O} 11-\mathrm{Tm}-\mathrm{O} 7$ & $140.36(7)$ & $\mathrm{O} 10-\mathrm{O} 8-\mathrm{O} 1$ & $63.30(7)$ \\
\hline $\mathrm{O} 10-\mathrm{Tm}-\mathrm{O} 7$ & $80.06(7)$ & $\mathrm{N} 3-\mathrm{O} 8-\mathrm{O} 13$ & $100.19(13)$ \\
\hline $\mathrm{O} 12-\mathrm{Tm}-\mathrm{O} 13$ & $139.17(6)$ & $\mathrm{O} 7-\mathrm{O} 8-\mathrm{O} 13$ & $78.06(8)$ \\
\hline $\mathrm{O} 11-\mathrm{Tm}-\mathrm{O} 13$ & $137.72(6)$ & $\mathrm{O} 9-\mathrm{O} 8-\mathrm{O} 13$ & $117.62(10)$ \\
\hline $\mathrm{O} 10-\mathrm{Tm}-\mathrm{O} 13$ & $133.41(6)$ & $\mathrm{Tm}-\mathrm{O} 8-\mathrm{O} 13$ & $53.17(5)$ \\
\hline $\mathrm{O} 7-\mathrm{Tm}-\mathrm{O} 13$ & $81.90(6)$ & $\mathrm{O} 10-\mathrm{O} 8-\mathrm{O} 13$ & $104.61(7)$ \\
\hline $\mathrm{O} 12-\mathrm{Tm}-\mathrm{O} 4$ & $81.26(7)$ & $\mathrm{O} 1-\mathrm{O} 8-\mathrm{O} 13$ & $66.39(6)$ \\
\hline $\mathrm{O} 11-\mathrm{Tm}-\mathrm{O} 4$ & $71.73(6)$ & $\mathrm{N} 3-\mathrm{O} 8-\mathrm{O} 3^{\mathrm{iv}}$ & $127.07(15)$ \\
\hline $\mathrm{O} 10-\mathrm{Tm}-\mathrm{O} 4$ & $139.74(7)$ & $\mathrm{O} 7-\mathrm{O} 8-\mathrm{O} 3^{\mathrm{iv}}$ & $130.96(11)$ \\
\hline $\mathrm{O} 7-\mathrm{Tm}-\mathrm{O} 4$ & $120.66(6)$ & $\mathrm{O} 9-\mathrm{O} 8-\mathrm{O}^{\mathrm{iv}}$ & $113.90(10)$ \\
\hline $\mathrm{O} 13-\mathrm{Tm}-\mathrm{O} 4$ & $85.91(6)$ & $\mathrm{Tm}-\mathrm{O} 8-\mathrm{O} 3^{\mathrm{iv}}$ & $107.97(7)$ \\
\hline $\mathrm{O} 12-\mathrm{Tm}-\mathrm{O} 1$ & $142.71(7)$ & $\mathrm{O} 10-\mathrm{O} 8-\mathrm{O}^{\mathrm{iv}}$ & $137.66(9)$ \\
\hline $\mathrm{O} 11-\mathrm{Tm}-\mathrm{O} 1$ & $80.83(6)$ & $\mathrm{O} 1-\mathrm{O} 8-\mathrm{O}^{\mathrm{iv}}$ & $74.92(7)$ \\
\hline $\mathrm{O} 10-\mathrm{Tm}-\mathrm{O} 1$ & 73.77 (7) & $\mathrm{O} 13-\mathrm{O} 8-\mathrm{O}^{\mathrm{iv}}$ & $61.63(7)$ \\
\hline $\mathrm{O} 7-\mathrm{Tm}-\mathrm{O} 1$ & $117.47(5)$ & $\mathrm{N} 3-\mathrm{O} 8-\mathrm{O} 14$ & $97.81(13)$ \\
\hline $\mathrm{O} 13-\mathrm{Tm}-\mathrm{O} 1$ & $77.34(6)$ & $\mathrm{O} 7-\mathrm{O} 8-\mathrm{O} 14$ & $130.15(10)$ \\
\hline $\mathrm{O} 4-\mathrm{Tm}-\mathrm{O} 1$ & $115.79(6)$ & $\mathrm{O} 9-\mathrm{O} 8-\mathrm{O} 14$ & $70.27(8)$ \\
\hline $\mathrm{O} 12-\mathrm{Tm}-\mathrm{O} 5$ & $72.69(6)$ & $\mathrm{Tm}-\mathrm{O} 8-\mathrm{O} 14$ & $168.88(9)$ \\
\hline $\mathrm{O} 11-\mathrm{Tm}-\mathrm{O} 5$ & $116.78(6)$ & $\mathrm{O} 10-\mathrm{O} 8-\mathrm{O} 14$ & $129.72(9)$ \\
\hline $\mathrm{O} 10-\mathrm{Tm}-\mathrm{O} 5$ & $139.64(7)$ & $\mathrm{O} 1-\mathrm{O} 8-\mathrm{O} 14$ & $115.20(8)$ \\
\hline $\mathrm{O} 7-\mathrm{Tm}-\mathrm{O} 5$ & $69.91(6)$ & $\mathrm{O} 13-\mathrm{O} 8-\mathrm{O} 14$ & $120.95(9)$ \\
\hline $\mathrm{O} 13-\mathrm{Tm}-\mathrm{O} 5$ & $69.01(6)$ & $\mathrm{O}^{\mathrm{iv}}-\mathrm{O} 8-\mathrm{O} 14$ & $62.67(8)$ \\
\hline $\mathrm{O} 4-\mathrm{Tm}-\mathrm{O} 5$ & $51.62(5)$ & $\mathrm{N} 3-\mathrm{O} 9-\mathrm{O} 8$ & $28.84(12)$ \\
\hline $\mathrm{O} 1-\mathrm{Tm}-\mathrm{O} 5$ & $144.29(6)$ & $\mathrm{N} 3-\mathrm{O} 9-\mathrm{O} 7$ & $30.33(11)$ \\
\hline $\mathrm{O} 12-\mathrm{Tm}-\mathrm{O} 8$ & $113.85(6)$ & $\mathrm{O} 8-\mathrm{O} 9-\mathrm{O} 7$ & $59.18(7)$ \\
\hline
\end{tabular}




$$
\begin{aligned}
& \mathrm{O} 11-\mathrm{Tm}-\mathrm{O} 8 \\
& \mathrm{O} 10-\mathrm{Tm}-\mathrm{O} 8 \\
& \text { O7-Tm-O8 } \\
& \mathrm{O} 13-\mathrm{Tm}-\mathrm{O} 8 \\
& \text { O4- Tm-O8 } \\
& \mathrm{O} 1-\mathrm{Tm}-\mathrm{O} 8 \\
& \text { O5- Tm-O8 } \\
& \mathrm{O} 12-\mathrm{Tm}-\mathrm{O} 2 \\
& \mathrm{O} 11-\mathrm{Tm}-\mathrm{O} 2 \\
& \mathrm{O} 10-\mathrm{Tm}-\mathrm{O} 2 \\
& \mathrm{O} 7-\mathrm{Tm}-\mathrm{O} 2 \\
& \mathrm{O} 13-\mathrm{Tm}-\mathrm{O} 2 \\
& \mathrm{O} 4-\mathrm{Tm}-\mathrm{O} 2 \\
& \mathrm{O} 1-\mathrm{Tm}-\mathrm{O} 2 \\
& \mathrm{O} 5-\mathrm{Tm}-\mathrm{O} 2 \\
& \mathrm{O} 8-\mathrm{Tm}-\mathrm{O} 2 \\
& \text { O12-Tm-N2 } \\
& \text { O11-Tm-N2 } \\
& \mathrm{O} 10-\mathrm{Tm}-\mathrm{N} 2 \\
& \text { O7- Tm-N2 } \\
& \mathrm{O} 13-\mathrm{Tm}-\mathrm{N} 2 \\
& \mathrm{O} 4-\mathrm{Tm}-\mathrm{N} 2 \\
& \mathrm{O} 1-\mathrm{Tm}-\mathrm{N} 2 \\
& \text { O5- Tm- N2 } \\
& \mathrm{O} 8-\mathrm{Tm}-\mathrm{N} 2 \\
& \mathrm{O} 2-\mathrm{Tm}-\mathrm{N} 2 \\
& \mathrm{O} 12-\mathrm{Tm}-\mathrm{N} 3 \\
& \text { O11-Tm-N3 } \\
& \text { O10-Tm-N3 } \\
& \text { O7- Tm-N3 } \\
& \text { O13-Tm-N3 } \\
& \text { O4- Tm-N3 } \\
& \mathrm{O} 1-\mathrm{Tm}-\mathrm{N} 3 \\
& \text { O5- Tm- N3 } \\
& \mathrm{O} 8-\mathrm{Tm}-\mathrm{N} 3 \\
& \mathrm{O} 2-\mathrm{Tm}-\mathrm{N} 3 \\
& \mathrm{~N} 2-\mathrm{Tm}-\mathrm{N} 3 \\
& \text { O12-Tm-N1 } \\
& \text { O11-Tm-N1 } \\
& \text { O10-Tm-N1 } \\
& \text { O7- Tm-N1 } \\
& \text { O13-Tm-N1 } \\
& \text { O4- Tm-N1 } \\
& \mathrm{O} 1-\mathrm{Tm}-\mathrm{N} 1 \\
& \text { O5- Tm-N1 } \\
& \mathrm{O} 8-\mathrm{Tm}-\mathrm{N} 1 \\
& \mathrm{O} 2-\mathrm{Tm}-\mathrm{N} 1 \\
& \mathrm{~N} 2-\mathrm{Tm}-\mathrm{N} 1
\end{aligned}
$$

\begin{tabular}{|c|c|c|}
\hline $132.83(6)$ & $\mathrm{N} 3-\mathrm{O} 9-\mathrm{O} 14$ & $95.93(15)$ \\
\hline $67.31(6)$ & $\mathrm{O} 8-\mathrm{O} 9-\mathrm{O} 14$ & $67.39(8)$ \\
\hline $51.11(5)$ & $\mathrm{O} 7-\mathrm{O} 9-\mathrm{O} 14$ & $125.90(11)$ \\
\hline $67.90(6)$ & $\mathrm{N} 3-\mathrm{O} 9-\mathrm{O}^{\mathrm{v}}$ & $76.99(16)$ \\
\hline $152.92(6)$ & $\mathrm{O} 8-\mathrm{O} 9-\mathrm{O} 6^{\mathrm{v}}$ & $82.55(9)$ \\
\hline $66.42(5)$ & $\mathrm{O} 7-\mathrm{O} 9-\mathrm{O}^{\mathrm{v}}$ & $74.67(9)$ \\
\hline $109.44(5)$ & $\mathrm{O} 14-\mathrm{O} 9-\mathrm{O}^{\mathrm{v}}$ & $91.35(9)$ \\
\hline $136.38(6)$ & $\mathrm{N} 3-\mathrm{O} 9-\mathrm{O} 6^{\mathrm{viii}}$ & $152.81(17)$ \\
\hline $69.47(6)$ & $\mathrm{O} 8-\mathrm{O} 9-\mathrm{O}^{\mathrm{viii}}$ & $124.77(10)$ \\
\hline $114.60(6)$ & $\mathrm{O} 7-\mathrm{O} 9-\mathrm{O} 6^{\text {viii }}$ & $170.46(13)$ \\
\hline $149.67(6)$ & $\mathrm{O} 14-09-\mathrm{O}^{\mathrm{viii}}$ & $60.20(7)$ \\
\hline $68.73(6)$ & $\mathrm{O}^{\mathrm{v}}-\mathrm{O} 9-\mathrm{O}^{\mathrm{viii}}$ & $113.72(8)$ \\
\hline $66.13(6)$ & $\mathrm{Tm}-\mathrm{O} 10-\mathrm{O} 11$ & $53.69(5)$ \\
\hline $49.93(5)$ & $\mathrm{Tm}-\mathrm{O} 10-\mathrm{O} 8$ & $60.27(6)$ \\
\hline $104.73(6)$ & $\mathrm{O} 11-\mathrm{O} 10-\mathrm{O} 8$ & $110.30(8)$ \\
\hline $108.01(5)$ & $\mathrm{Tm}-\mathrm{O} 10-\mathrm{O} 12$ & $53.11(5)$ \\
\hline $75.56(6)$ & $\mathrm{O} 11-\mathrm{O} 10-\mathrm{O} 12$ & $60.49(6)$ \\
\hline $94.20(6)$ & $\mathrm{O} 8-\mathrm{O} 10-\mathrm{O} 12$ & $96.36(7)$ \\
\hline $147.88(7)$ & $\mathrm{Tm}-\mathrm{O} 10-\mathrm{O} 1^{\mathrm{i}}$ & $130.59(9)$ \\
\hline $95.49(6)$ & $\mathrm{O} 11-\mathrm{O} 10-\mathrm{O} 1^{\mathrm{i}}$ & $143.50(9)$ \\
\hline $76.27(6)$ & $\mathrm{O} 8-\mathrm{O} 10-\mathrm{O} 1^{\mathrm{i}}$ & $73.51(7)$ \\
\hline $25.62(6)$ & $\mathrm{O} 12-\mathrm{O} 10-\mathrm{O} 1^{\mathrm{i}}$ & $155.76(9)$ \\
\hline $133.73(6)$ & $\mathrm{Tm}-\mathrm{O} 10-\mathrm{O} 1$ & $54.63(5)$ \\
\hline $26.00(6)$ & $\mathrm{O} 11-\mathrm{O} 10-\mathrm{O} 1$ & $66.79(7)$ \\
\hline $132.96(6)$ & $\mathrm{O} 8-\mathrm{O} 10-\mathrm{O} 1$ & $58.62(6)$ \\
\hline $85.07(6)$ & $\mathrm{O} 12-\mathrm{O} 10-\mathrm{O} 1$ & $106.09(8)$ \\
\hline $92.45(6)$ & $\mathrm{O} 1-\mathrm{i} 10-\mathrm{O} 1$ & $87.67(7)$ \\
\hline $142.15(6)$ & $\mathrm{Tm}-\mathrm{O} 10-\mathrm{O} 13^{\mathrm{ix}}$ & $122.60(8)$ \\
\hline $70.66(6)$ & $\mathrm{O} 11-\mathrm{O} 10-\mathrm{O} 13^{\mathrm{ix}}$ & $128.05(9)$ \\
\hline $25.75(6)$ & $\mathrm{O} 8-\mathrm{O} 10-\mathrm{O} 13^{\mathrm{ix}}$ & $103.81(7)$ \\
\hline $74.57(6)$ & $\mathrm{O} 12-\mathrm{O} 10-\mathrm{O} 13^{\mathrm{ix}}$ & $78.12(7)$ \\
\hline $141.95(6)$ & $\mathrm{O} 1^{\mathrm{i}}-\mathrm{O} 10-\mathrm{O} 13^{\mathrm{ix}}$ & $82.96(7)$ \\
\hline $91.72(5)$ & $\mathrm{O} 1-\mathrm{O} 10-\mathrm{O} 13^{\mathrm{ix}}$ & $162.00(8)$ \\
\hline $90.60(5)$ & $\mathrm{Tm}-\mathrm{O} 10-\mathrm{O} 2^{\mathrm{ix}}$ & $135.89(8)$ \\
\hline $25.43(5)$ & $\mathrm{O} 11-\mathrm{O} 10-\mathrm{O} 2^{\mathrm{ix}}$ & $90.74(7)$ \\
\hline $131.08(5)$ & $\mathrm{O} 8-\mathrm{O} 10-\mathrm{O} 2^{\mathrm{ix}}$ & $158.09(8)$ \\
\hline $116.50(6)$ & $\mathrm{O} 12-\mathrm{O} 10-\mathrm{O} 2^{\mathrm{ix}}$ & $88.57(7)$ \\
\hline $145.99(6)$ & $\mathrm{O} 1^{\mathrm{i}}-\mathrm{O} 10-\mathrm{O} 2^{\mathrm{ix}}$ & $93.44(7)$ \\
\hline $72.73(6)$ & $\mathrm{O} 1-\mathrm{O} 10-\mathrm{O} 2^{\mathrm{ix}}$ & $140.06(8)$ \\
\hline $93.76(6)$ & $\mathrm{O} 13^{\mathrm{ix}}-\mathrm{O} 10-\mathrm{O} 2^{\mathrm{ix}}$ & $56.26(6)$ \\
\hline $137.60(5)$ & $\mathrm{Tm}-\mathrm{O} 10-\mathrm{O} 7$ & $50.62(5)$ \\
\hline $72.24(6)$ & $\mathrm{O} 11-\mathrm{O} 10-\mathrm{O} 7$ & $100.01(7)$ \\
\hline $90.89(6)$ & $\mathrm{O} 8-\mathrm{O} 10-\mathrm{O} 7$ & $43.18(5)$ \\
\hline $24.96(5)$ & $\mathrm{O} 12-\mathrm{O} 10-\mathrm{O} 7$ & $56.67(6)$ \\
\hline $126.60(6)$ & $\mathrm{O} 1-\mathrm{O} 10-\mathrm{O} 7$ & $105.10(7)$ \\
\hline $87.63(5)$ & $\mathrm{O} 1-\mathrm{O} 10-\mathrm{O} 7$ & $88.42(7)$ \\
\hline $25.01(5)$ & $\mathrm{O} 13^{\mathrm{ix}}-\mathrm{O} 10-\mathrm{O} 7$ & $79.30(7)$ \\
\hline $109.73(6)$ & $\mathrm{O} 2^{\mathrm{ix}}-\mathrm{O} 10-\mathrm{O} 7$ & $129.22(8)$ \\
\hline
\end{tabular}




\begin{tabular}{|c|c|c|c|}
\hline $\mathrm{N} 3-\mathrm{Tm}-\mathrm{N} 1$ & $112.83(5)$ & $\mathrm{Tm}-\mathrm{O} 10-\mathrm{H} 1$ & $133(3)$ \\
\hline $\mathrm{O} 3-\mathrm{N} 1-\mathrm{O} 2$ & $122.7(2)$ & $\mathrm{O} 11-\mathrm{O} 10-\mathrm{H} 1$ & $140(2)$ \\
\hline $\mathrm{O} 3-\mathrm{N} 1-\mathrm{O} 1$ & $121.65(19)$ & $\mathrm{O} 8-\mathrm{O} 10-\mathrm{H} 1$ & $77(3)$ \\
\hline $\mathrm{O} 2-\mathrm{N} 1-\mathrm{O} 1$ & $115.65(18)$ & $\mathrm{O} 12-\mathrm{O} 10-\mathrm{H} 1$ & $159(2)$ \\
\hline $\mathrm{O} 3-\mathrm{N} 1-\mathrm{Tm}$ & $175.24(17)$ & 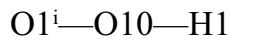 & $5(2)$ \\
\hline $\mathrm{O} 2-\mathrm{N} 1-\mathrm{Tm}$ & $61.74(11)$ & $\mathrm{O} 1-\mathrm{O} 10-\mathrm{H} 1$ & $88(3)$ \\
\hline $\mathrm{O} 1-\mathrm{N} 1-\mathrm{Tm}$ & $54.03(10)$ & $\mathrm{O} 13^{\mathrm{ix}}-\mathrm{O} 10-\mathrm{H} 1$ & $84(3)$ \\
\hline $\mathrm{N} 1-\mathrm{O} 1-\mathrm{O} 2$ & $31.90(10)$ & $\mathrm{O} 2^{\mathrm{ix}}-\mathrm{O} 10-\mathrm{H} 1$ & $91(3)$ \\
\hline $\mathrm{N} 1-\mathrm{O} 1-\mathrm{O} 3$ & $28.46(11)$ & $\mathrm{O} 7-\mathrm{O} 10-\mathrm{H} 1$ & $110(2)$ \\
\hline $\mathrm{O} 2-\mathrm{O} 1-\mathrm{O} 3$ & $60.35(8)$ & $\mathrm{Tm}-\mathrm{O} 10-\mathrm{H} 2$ & $117(2)$ \\
\hline $\mathrm{N} 1-\mathrm{O} 1-\mathrm{Tm}$ & $101.01(12)$ & $\mathrm{O} 11-\mathrm{O} 10-\mathrm{H} 2$ & $103(2)$ \\
\hline $\mathrm{O} 2-\mathrm{O} 1-\mathrm{Tm}$ & $69.19(6)$ & $\mathrm{O} 8-\mathrm{O} 10-\mathrm{H} 2$ & $125(3)$ \\
\hline $\mathrm{O} 3-\mathrm{O} 1-\mathrm{Tm}$ & $129.44(9)$ & $\mathrm{O} 12-\mathrm{O} 10-\mathrm{H} 2$ & $64(2)$ \\
\hline $\mathrm{N} 1-\mathrm{O} 1-\mathrm{O} 8$ & $142.91(14)$ & $\mathrm{O} 1{ }^{\mathrm{i}-\mathrm{O} 10-\mathrm{H} 2}$ & $103(2)$ \\
\hline $\mathrm{O} 2-\mathrm{O} 1-\mathrm{O} 8$ & $117.71(9)$ & $\mathrm{O} 1-\mathrm{O} 10-\mathrm{H} 2$ & $169(2)$ \\
\hline $\mathrm{O} 3-\mathrm{O} 1-\mathrm{O} 8$ & $152.42(12)$ & $\mathrm{O} 13^{\mathrm{ix}}-\mathrm{O} 10-\mathrm{H} 2$ & $26(2)$ \\
\hline $\mathrm{Tm}-\mathrm{O} 1-\mathrm{O} 8$ & $59.05(5)$ & $\mathrm{O} 2^{\mathrm{ix}}-\mathrm{O} 10-\mathrm{H} 2$ & $39(3)$ \\
\hline $\mathrm{N} 1-\mathrm{O} 1-\mathrm{O} 10^{\mathrm{i}}$ & $125.68(13)$ & $\mathrm{O} 7-\mathrm{O} 10-\mathrm{H} 2$ & $90(3)$ \\
\hline $\mathrm{O} 2-\mathrm{O} 1-\mathrm{O} 10^{\mathrm{i}}$ & $155.65(10)$ & $\mathrm{H} 1-\mathrm{O} 10-\mathrm{H} 2$ & $103(4)$ \\
\hline $\mathrm{O} 3-\mathrm{O} 1-\mathrm{O} 10^{\mathrm{i}}$ & 97.77 (9) & $\mathrm{Tm}-\mathrm{O} 11-\mathrm{O} 10$ & $54.68(5)$ \\
\hline $\mathrm{Tm}-\mathrm{O} 1-\mathrm{O} 10^{\mathrm{i}}$ & $132.31(7)$ & $\mathrm{Tm}-\mathrm{O} 11-\mathrm{O} 14^{\mathrm{i}}$ & $126.09(8)$ \\
\hline $\mathrm{O} 8-\mathrm{O} 1-\mathrm{O} 10^{\mathrm{i}}$ & $76.13(6)$ & $\mathrm{O} 10-\mathrm{O} 11-\mathrm{O} 14^{\mathrm{i}}$ & $79.46(8)$ \\
\hline $\mathrm{N} 1-\mathrm{O} 1-\mathrm{O} 10$ & $136.42(14)$ & $\mathrm{Tm}-\mathrm{O} 11-\mathrm{O} 12$ & $53.31(5)$ \\
\hline $\mathrm{O} 2-\mathrm{O} 1-\mathrm{O} 10$ & $111.95(9)$ & $\mathrm{O} 10-\mathrm{O} 11-\mathrm{O} 12$ & $60.44(6)$ \\
\hline $\mathrm{O} 3-\mathrm{O} 1-\mathrm{O} 10$ & $149.48(11)$ & $\mathrm{O} 14^{\mathrm{i}}-\mathrm{O} 11-\mathrm{O} 12$ & $128.28(10)$ \\
\hline $\mathrm{Tm}-\mathrm{O} 1-\mathrm{O} 10$ & $51.60(5)$ & $\mathrm{Tm}-\mathrm{O} 11-\mathrm{O} 4$ & $55.71(5)$ \\
\hline $\mathrm{O} 8-\mathrm{O} 1-\mathrm{O} 10$ & $58.08(6)$ & $\mathrm{O} 10-\mathrm{O} 11-\mathrm{O} 4$ & $108.60(8)$ \\
\hline $\mathrm{O} 10-\mathrm{O} 1-\mathrm{O} 10$ & $92.33(7)$ & $\mathrm{O} 14-\mathrm{O} 11-\mathrm{O} 4$ & $163.22(11)$ \\
\hline $\mathrm{N} 1-\mathrm{O} 1-\mathrm{O} 13$ & 85.66 (12) & $\mathrm{O} 12-\mathrm{O} 11-\mathrm{O} 4$ & $67.54(7)$ \\
\hline $\mathrm{O} 2-\mathrm{O} 1-\mathrm{O} 13$ & $63.91(7)$ & $\mathrm{Tm}-\mathrm{O} 11-\mathrm{O} 2$ & $60.20(5)$ \\
\hline $\mathrm{O} 3-\mathrm{O} 1-\mathrm{O} 13$ & $105.20(10)$ & $\mathrm{O} 10-\mathrm{O} 11-\mathrm{O} 2$ & $97.60(7)$ \\
\hline $\mathrm{Tm}-\mathrm{O} 1-\mathrm{O} 13$ & $50.75(5)$ & $\mathrm{O} 14-\mathrm{O} 11-\mathrm{O} 2$ & $106.22(9)$ \\
\hline $\mathrm{O} 8-\mathrm{O} 1-\mathrm{O} 13$ & $57.34(6)$ & $\mathrm{O} 12-\mathrm{O} 11-\mathrm{O} 2$ & $110.01(8)$ \\
\hline $\mathrm{O} 10-\mathrm{O} 1-\mathrm{O} 13$ & $117.55(8)$ & $\mathrm{O} 4-\mathrm{O} 11-\mathrm{O} 2$ & $58.83(7)$ \\
\hline $\mathrm{O} 10-\mathrm{O} 1-\mathrm{O} 13$ & $95.28(7)$ & $\mathrm{Tm}-\mathrm{O} 11-\mathrm{O} 2^{\mathrm{ii}}$ & $128.10(9)$ \\
\hline $\mathrm{N} 1-\mathrm{O} 1-\mathrm{O} 11$ & 82.07 (12) & $\mathrm{O} 10-\mathrm{O} 11-\mathrm{O} 2^{\mathrm{ii}}$ & $169.77(9)$ \\
\hline $\mathrm{O} 2-\mathrm{O} 1-\mathrm{O} 11$ & $62.22(7)$ & $\mathrm{O} 14-\mathrm{O} 11-\mathrm{O} 2^{\mathrm{ii}}$ & $102.25(8)$ \\
\hline $\mathrm{O} 3-\mathrm{O} 1-\mathrm{O} 11$ & $101.73(9)$ & $\mathrm{O} 12-\mathrm{O} 11-\mathrm{O} 2^{\mathrm{ii}}$ & $112.15(8)$ \\
\hline $\mathrm{Tm}-\mathrm{O} 1-\mathrm{O} 11$ & $47.83(4)$ & $\mathrm{O} 4-\mathrm{O} 11-\mathrm{O} 2^{\mathrm{ii}}$ & $72.52(7)$ \\
\hline $\mathrm{O} 8-\mathrm{O} 1-\mathrm{O} 11$ & $100.20(7)$ & $\mathrm{O} 2-\mathrm{O} 11-\mathrm{O} 2^{\mathrm{ii}}$ & $91.62(7)$ \\
\hline $\mathrm{O} 10-\mathrm{O} 1-\mathrm{O} 11$ & $138.47(9)$ & $\mathrm{Tm}-\mathrm{O} 11-\mathrm{O} 1$ & $51.34(5)$ \\
\hline $\mathrm{O} 10-\mathrm{O} 1-\mathrm{O} 11$ & $54.35(6)$ & $\mathrm{O} 10-\mathrm{O} 11-\mathrm{O} 1$ & $58.86(6)$ \\
\hline $\mathrm{O} 13-\mathrm{O} 1-\mathrm{O} 11$ & $92.12(6)$ & $\mathrm{O} 14-\mathrm{O} 11-\mathrm{O} 1$ & $82.73(7)$ \\
\hline $\mathrm{N} 1-\mathrm{O} 2-\mathrm{O} 1$ & $32.45(11)$ & $\mathrm{O} 12-\mathrm{O} 11-\mathrm{O} 1$ & $100.57(7)$ \\
\hline $\mathrm{N} 1-\mathrm{O} 2-\mathrm{O} 3$ & $28.19(11)$ & $\mathrm{O} 4-\mathrm{O} 11-\mathrm{O} 1$ & $88.96(7)$ \\
\hline $\mathrm{O} 1-\mathrm{O} 2-\mathrm{O} 3$ & $60.63(8)$ & $\mathrm{O} 2-\mathrm{O} 11-\mathrm{O} 1$ & $42.16(5)$ \\
\hline $\mathrm{N} 1-\mathrm{O} 2-\mathrm{Tm}$ & 93.26 (12) & $\mathrm{O} 2^{\mathrm{ii}}-\mathrm{O} 11-\mathrm{O} 1$ & $131.25(8)$ \\
\hline $\mathrm{O} 1-\mathrm{O} 2-\mathrm{Tm}$ & $60.88(6)$ & $\mathrm{Tm}-\mathrm{O} 11-\mathrm{O} 4^{\mathrm{ii}}$ & $126.48(8)$ \\
\hline
\end{tabular}




$$
\begin{aligned}
& \mathrm{O} 3-\mathrm{O} 2-\mathrm{Tm} \\
& \mathrm{N} 1-\mathrm{O} 2-\mathrm{O} 4 \\
& \mathrm{O} 1-\mathrm{O} 2-\mathrm{O} 4 \\
& \mathrm{O} 3-\mathrm{O} 2-\mathrm{O} 4 \\
& \mathrm{Tm}-\mathrm{O} 2-\mathrm{O} 4 \\
& \mathrm{~N} 1-\mathrm{O} 2-\mathrm{O} 11 \\
& \mathrm{O} 1-\mathrm{O} 2-\mathrm{O} 11 \\
& \mathrm{O} 3-\mathrm{O} 2-\mathrm{O} 11 \\
& \mathrm{Tm}-\mathrm{O} 2-\mathrm{O} 11 \\
& \mathrm{O} 4-\mathrm{O} 2-\mathrm{O} 11 \\
& \mathrm{~N} 1-\mathrm{O} 2-\mathrm{O} 13 \\
& \mathrm{O} 1-\mathrm{O} 2-\mathrm{O} 13 \\
& \mathrm{O} 3-\mathrm{O} 2-\mathrm{O} 13 \\
& \mathrm{Tm}-\mathrm{O} 2-\mathrm{O} 13 \\
& \mathrm{O} 4-\mathrm{O} 2-\mathrm{O} 13 \\
& \mathrm{O} 11-\mathrm{O} 2-\mathrm{O} 13 \\
& \mathrm{~N} 1-\mathrm{O} 2-\mathrm{O} 11^{\mathrm{ii}} \\
& \mathrm{O} 1-\mathrm{O} 2-\mathrm{O} 11^{\mathrm{ii}} \\
& \mathrm{O} 3-\mathrm{O} 2-\mathrm{O} 11^{\mathrm{ii}} \\
& \mathrm{Tm}-\mathrm{O} 2-\mathrm{O} 11^{\mathrm{ii}} \\
& \mathrm{O} 4-\mathrm{O} 2-\mathrm{O} 11^{\mathrm{ii}} \\
& \mathrm{O} 11-\mathrm{O} 2-\mathrm{O} 11^{\mathrm{ii}} \\
& \mathrm{O} 13-\mathrm{O} 2-\mathrm{O} 11^{\mathrm{ii}} \\
& \mathrm{N} 1-\mathrm{O} 2-\mathrm{O} 10^{\mathrm{iii}} \\
& \mathrm{O} 1-\mathrm{O} 2-\mathrm{O} 10^{\mathrm{iii}} \\
& \mathrm{O} 3-\mathrm{O} 2-\mathrm{O} 10^{\mathrm{iii}} \\
& \mathrm{Tm}-\mathrm{O} 2-\mathrm{O} 10^{\mathrm{iii}} \\
& \mathrm{O} 4-\mathrm{O} 2-\mathrm{O} 10^{\mathrm{iii}} \\
& \mathrm{O} 11-\mathrm{O} 2-\mathrm{O} 10^{\mathrm{iii}} \\
& \mathrm{O} 13-\mathrm{O} 2-\mathrm{O} 10^{\mathrm{iii}} \\
& \mathrm{O} 11^{\mathrm{ii}}-\mathrm{O} 2-\mathrm{O} 10^{\mathrm{iii}} \\
& \mathrm{N} 1-\mathrm{O} 3-\mathrm{O} 2 \\
& \mathrm{~N} 1-\mathrm{O} 3-\mathrm{O} 1 \\
& \mathrm{O} 2-\mathrm{O} 3-\mathrm{O} 1 \\
& \mathrm{~N} 1-\mathrm{O} 3-\mathrm{O} 13^{\mathrm{iv}} \\
& \mathrm{O} 2-\mathrm{O} 3-\mathrm{O} 13^{\mathrm{iv}} \\
& \mathrm{O} 1-\mathrm{O} 3-\mathrm{O} 13^{\mathrm{iv}} \\
& \mathrm{N} 1-\mathrm{O} 3-\mathrm{O} 8^{\mathrm{iv}} \\
& \mathrm{O} 2-\mathrm{O} 3-\mathrm{O} 8^{\mathrm{iv}} \\
& \mathrm{O} 1-\mathrm{O} 3-\mathrm{O} 8^{\mathrm{iv}} \\
& \mathrm{O} 13^{\mathrm{iv}}-\mathrm{O} 3-\mathrm{O} 8^{\mathrm{iv}} \\
& \mathrm{N} 1-\mathrm{O} 3-\mathrm{O} 14^{\mathrm{iv}} \\
& \mathrm{O} 2-\mathrm{O} 3-\mathrm{O} 14^{\mathrm{iv}} \\
& \mathrm{O} 1-\mathrm{O} 3-\mathrm{O} 14^{\mathrm{iv}} \\
& \mathrm{O} 13-\mathrm{iv} 3-\mathrm{O} 14^{\mathrm{iv}} \\
& \mathrm{O} 8-\mathrm{O} 3-\mathrm{O} 14^{\mathrm{iv}} \\
& \mathrm{O} 6-\mathrm{N} 2-\mathrm{O} 4 \\
& \mathrm{O} 6-\mathrm{N} 2-\mathrm{O} 5 \\
&
\end{aligned}
$$

$121.43(9)$

\begin{tabular}{|c|c|}
\hline $\mathrm{O} 10-\mathrm{O} 11-\mathrm{O} 4^{\mathrm{ii}}$ & $135.39(8)$ \\
\hline $\mathrm{O} 14^{\mathrm{i}}-\mathrm{O} 11-\mathrm{O} 4^{\mathrm{ii}}$ & $67.19(8)$ \\
\hline $\mathrm{O} 12-\mathrm{O} 11-\mathrm{O} 4^{\mathrm{ii}}$ & $163.11(9)$ \\
\hline $\mathrm{O} 4-\mathrm{O} 11-\mathrm{O} 4^{\mathrm{ii}}$ & $98.00(7)$ \\
\hline $\mathrm{O} 2-\mathrm{O} 11-\mathrm{O} 4^{\mathrm{ii}}$ & $66.31(6)$ \\
\hline $\mathrm{O} 2^{\mathrm{ii}}-\mathrm{O} 11-\mathrm{O} 4^{\mathrm{ii}}$ & $52.98(5)$ \\
\hline $\mathrm{O} 1-\mathrm{O} 11-\mathrm{O} 4^{\mathrm{ii}}$ & $87.35(6)$ \\
\hline $\mathrm{Tm}-\mathrm{O} 11-\mathrm{H} 3$ & $125(3)$ \\
\hline $\mathrm{O} 10-\mathrm{O} 11-\mathrm{H} 3$ & $75(3)$ \\
\hline $\mathrm{O} 14-\mathrm{O} 11-\mathrm{H} 3$ & $9(3)$ \\
\hline $\mathrm{O} 12-\mathrm{O} 11-\mathrm{H} 3$ & $120(3)$ \\
\hline $\mathrm{O} 4-\mathrm{O} 11-\mathrm{H} 3$ & $172(3)$ \\
\hline $\mathrm{O} 2-\mathrm{O} 11-\mathrm{H} 3$ & $114(3)$ \\
\hline $\mathrm{O} 2^{\mathrm{ii}}-\mathrm{O} 11-\mathrm{H} 3$ & $105(3)$ \\
\hline $\mathrm{O} 1-\mathrm{O} 11-\mathrm{H} 3$ & $87(3)$ \\
\hline $\mathrm{O} 44^{\mathrm{ii}}-\mathrm{O} 11-\mathrm{H} 3$ & $75(3)$ \\
\hline $\mathrm{Tm}-\mathrm{O} 11-\mathrm{H} 4$ & $122(3)$ \\
\hline $\mathrm{O} 10-\mathrm{O} 11-\mathrm{H} 4$ & $169(2)$ \\
\hline $\mathrm{O} 14-\mathrm{O} 11-\mathrm{H} 4$ & $108(2)$ \\
\hline $\mathrm{O} 12-\mathrm{O} 11-\mathrm{H} 4$ & $109(2)$ \\
\hline $\mathrm{O} 4-\mathrm{O} 11-\mathrm{H} 4$ & $66(2)$ \\
\hline $\mathrm{O} 2-\mathrm{O} 11-\mathrm{H} 4$ & $88(3)$ \\
\hline $\mathrm{O} 2^{\mathrm{ii}}-\mathrm{O} 11-\mathrm{H} 4$ & $6(3)$ \\
\hline $\mathrm{O} 1-\mathrm{O} 11-\mathrm{H} 4$ & $129(3)$ \\
\hline $\mathrm{O} 4^{\mathrm{ii}-\mathrm{O} 11-\mathrm{H} 4}$ & $56(2)$ \\
\hline $\mathrm{H} 3-\mathrm{O} 11-\mathrm{H} 4$ & $111(4)$ \\
\hline $\mathrm{Tm}-\mathrm{O} 12-\mathrm{O} 14^{\mathrm{x}}$ & $125.11(10)$ \\
\hline $\mathrm{Tm}-\mathrm{O} 12-\mathrm{O} 10$ & $54.08(5)$ \\
\hline $\mathrm{O} 14^{\mathrm{x}}-\mathrm{O} 12-\mathrm{O} 10$ & $116.16(9)$ \\
\hline $\mathrm{Tm}-\mathrm{O} 12-\mathrm{O} 7^{\mathrm{vii}}$ & $120.70(8)$ \\
\hline $\mathrm{O} 14^{\mathrm{x}}-\mathrm{O} 12-\mathrm{O} 7^{\mathrm{vii}}$ & $105.94(8)$ \\
\hline $\mathrm{O} 10-\mathrm{O} 12-\mathrm{O} 7^{\mathrm{vii}}$ & $128.10(8)$ \\
\hline $\mathrm{Tm}-\mathrm{O} 12-\mathrm{O} 11$ & $53.31(5)$ \\
\hline $\mathrm{O} 14^{\mathrm{x}}-\mathrm{O} 12-\mathrm{O} 11$ & $75.07(8)$ \\
\hline $\mathrm{O} 10-\mathrm{O} 12-\mathrm{O} 11$ & $59.06(6)$ \\
\hline $\mathrm{O} 7^{\mathrm{vii}}-\mathrm{O} 12-\mathrm{O} 11$ & $167.99(10)$ \\
\hline $\mathrm{Tm}-\mathrm{O} 12-\mathrm{O} 7$ & $55.21(5)$ \\
\hline $\mathrm{O} 14^{\mathrm{x}}-\mathrm{O} 12-\mathrm{O} 7$ & $176.85(10)$ \\
\hline $\mathrm{O} 10-\mathrm{O} 12-\mathrm{O} 7$ & $66.77(6)$ \\
\hline $\mathrm{O} 7{ }^{\mathrm{vii}}-\mathrm{O} 12-\mathrm{O} 7$ & $72.20(7)$ \\
\hline $\mathrm{O} 11-\mathrm{O} 12-\mathrm{O} 7$ & $106.19(8)$ \\
\hline $\mathrm{Tm}-\mathrm{O} 12-\mathrm{O} 5$ & $56.63(5)$ \\
\hline $\mathrm{O} 14^{\mathrm{x}}-\mathrm{O} 12-\mathrm{O} 5$ & $117.49(9)$ \\
\hline $\mathrm{O} 10-\mathrm{O} 12-\mathrm{O} 5$ & $107.92(8)$ \\
\hline $\mathrm{O} 7{ }^{\mathrm{vi}}-\mathrm{O} 12-\mathrm{O} 5$ & $75.17(7)$ \\
\hline $\mathrm{O} 11-\mathrm{O} 12-\mathrm{O} 5$ & $93.64(8)$ \\
\hline $\mathrm{O} 7-\mathrm{O} 12-\mathrm{O} 5$ & $59.73(6)$ \\
\hline $\mathrm{Tm}-\mathrm{O} 12-\mathrm{O} 4$ & $50.70(5)$ \\
\hline
\end{tabular}

$145.77(15)$

114.04 (9)

$170.50(12)$

$53.43(5)$

$94.41(14)$

$75.62(8)$

$110.77(10)$

$50.33(5)$

$59.72(7)$

$94.71(12)$

$73.45(7)$

111.95 (9)

$52.13(5)$

$71.96(6)$

$102.24(7)$

$135.37(13)$

$156.10(10)$

$110.89(9)$

$121.11(7)$

$70.59(6)$

$88.37(7)$

$128.19(8)$

$93.92(13)$

$103.88(9)$

$83.51(9)$

$113.27(7)$

$105.78(8)$

$162.02(8)$

$61.18(6)$

$96.80(7)$

$29.13(11)$

$29.90(11)$

$59.02(8)$

$123.70(15)$

$146.33(11)$

96.81 (9)

133.41 (18)

120.98 (12)

132.24 (11)

56.18 (6)

113.40 (16)

84.49 (9)

$142.73(10)$

112.24 (8)

58.87 (6)

122.98 (19)

122.2 (2) 


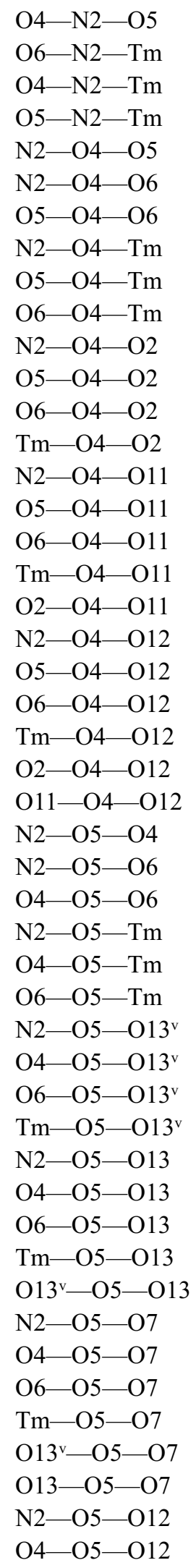

$114.76(17)$

$178.28(17)$

$55.33(10)$

$59.44(10)$

$32.70(10)$

27.78 (11)

60.48 (8)

$99.05(12)$

$66.35(6)$

126.84 (9)

$133.14(15)$

111.36 (9)

$141.71(12)$

$60.45(5)$

$140.81(15)$

112.74 (9)

$156.42(13)$

$52.55(5)$

61.45 (6)

84.97 (14)

$63.46(7)$

$104.30(10)$

48.04 (5)

$103.44(7)$

$56.19(6)$

32.54 (11)

28.07 (11)

$60.61(8)$

$94.56(12)$

62.03 (6)

$122.63(9)$

109.94 (13)

142.41 (9)

$81.90(8)$

155.31 (8)

$102.32(14)$

82.54 (8)

117.15 (11)

$53.82(5)$

115.30 (7)

$146.29(14)$

114.45 (9)

170.40 (11)

$53.21(5)$

$103.03(7)$

68.48 (6)

94.81 (14)

$74.50(8)$

\begin{tabular}{|c|c|}
\hline $\mathrm{O} 14^{\mathrm{x}}-\mathrm{O} 12-\mathrm{O} 4$ & $87.49(9)$ \\
\hline $\mathrm{O} 10-\mathrm{O} 12-\mathrm{O} 4$ & $99.49(7)$ \\
\hline $\mathrm{O} 7{ }^{\mathrm{vi}}-\mathrm{O} 12-\mathrm{O} 4$ & $111.72(8)$ \\
\hline $\mathrm{O} 11-\mathrm{O} 12-\mathrm{O} 4$ & $56.27(6)$ \\
\hline $\mathrm{O} 7-\mathrm{O} 12-\mathrm{O} 4$ & $90.85(7)$ \\
\hline $\mathrm{O} 5-\mathrm{O} 12-\mathrm{O} 4$ & $42.04(5)$ \\
\hline $\mathrm{Tm}-\mathrm{O} 12-\mathrm{O} 9^{\mathrm{vii}}$ & $131.81(9)$ \\
\hline $\mathrm{O} 14^{\mathrm{x}}-\mathrm{O} 12-\mathrm{O} 9^{\mathrm{vii}}$ & $70.05(7)$ \\
\hline $\mathrm{O} 10-\mathrm{O} 12-\mathrm{O}^{\mathrm{vii}}$ & $168.34(9)$ \\
\hline $\mathrm{O} 7^{\mathrm{vii}}-\mathrm{O} 12-\mathrm{O} 9^{\mathrm{vii}}$ & $41.15(5)$ \\
\hline $\mathrm{O} 11-\mathrm{O} 12-\mathrm{O}^{\mathrm{vii}}$ & $132.43(8)$ \\
\hline $\mathrm{O} 7-\mathrm{O} 12-\mathrm{O} 9^{\text {vii }}$ & $107.30(7)$ \\
\hline $\mathrm{O} 5-\mathrm{O} 12-\mathrm{O} 9^{\mathrm{vii}}$ & $75.57(7)$ \\
\hline $\mathrm{O} 4-\mathrm{O} 12-\mathrm{O} 9^{\mathrm{vii}}$ & $90.46(7)$ \\
\hline $\mathrm{Tm}-\mathrm{O} 12-\mathrm{H} 5$ & $123(3)$ \\
\hline $\mathrm{O} 14^{\mathrm{x}}-\mathrm{O} 12-\mathrm{H} 5$ & $10(3)$ \\
\hline $\mathrm{O} 10-\mathrm{O} 12-\mathrm{H} 5$ & $124(3)$ \\
\hline $\mathrm{O} 7^{\mathrm{vii}-\mathrm{O} 12-\mathrm{H} 5}$ & $102(3)$ \\
\hline $\mathrm{O} 11-\mathrm{O} 12-\mathrm{H} 5$ & $77(3)$ \\
\hline $\mathrm{O} 7-\mathrm{O} 12-\mathrm{H} 5$ & $166(3)$ \\
\hline $\mathrm{O} 5-\mathrm{O} 12-\mathrm{H} 5$ & $107(3)$ \\
\hline $\mathrm{O} 4-\mathrm{O} 12-\mathrm{H} 5$ & $80(3)$ \\
\hline $\mathrm{O} 9^{\mathrm{vii}}-\mathrm{O} 12-\mathrm{H} 5$ & $63(3)$ \\
\hline $\mathrm{Tm}-\mathrm{O} 12-\mathrm{H} 6$ & $118(3)$ \\
\hline $\mathrm{O} 14^{\mathrm{x}}-\mathrm{O} 12-\mathrm{H} 6$ & $107(2)$ \\
\hline $\mathrm{O} 10-\mathrm{O} 12-\mathrm{H} 6$ & $130(2)$ \\
\hline $\mathrm{O} 7{ }^{\mathrm{vii}}-\mathrm{O} 12-\mathrm{H} 6$ & $4(3)$ \\
\hline $\mathrm{O} 11-\mathrm{O} 12-\mathrm{H} 6$ & $164(3)$ \\
\hline $\mathrm{O} 7-\mathrm{O} 12-\mathrm{H} 6$ & $71(2)$ \\
\hline $\mathrm{O} 5-\mathrm{O} 12-\mathrm{H} 6$ & $71(3)$ \\
\hline $\mathrm{O} 4-\mathrm{O} 12-\mathrm{H} 6$ & $107(3)$ \\
\hline $\mathrm{O} 9^{\mathrm{vii}}-\mathrm{O} 12-\mathrm{H} 6$ & $40(2)$ \\
\hline $\mathrm{H} 5-\mathrm{O} 12-\mathrm{H} 6$ & $102(4)$ \\
\hline $\mathrm{Tm}-\mathrm{O} 13-\mathrm{O}^{\mathrm{v}}$ & $116.85(8)$ \\
\hline $\mathrm{Tm}-\mathrm{O} 13-\mathrm{O} 5$ & $57.18(5)$ \\
\hline $\mathrm{O} 5 \mathrm{v}-\mathrm{O} 13-\mathrm{O} 5$ & $64.70(7)$ \\
\hline $\mathrm{Tm}-\mathrm{O} 13-\mathrm{O} 8$ & $58.93(5)$ \\
\hline $\mathrm{O}^{\mathrm{v}}-\mathrm{O} 13-\mathrm{O} 8$ & $108.57(8)$ \\
\hline $\mathrm{O} 5-\mathrm{O} 13-\mathrm{O} 8$ & $96.27(7)$ \\
\hline $\mathrm{Tm}-\mathrm{O} 13-\mathrm{O} 2$ & $59.14(5)$ \\
\hline $\mathrm{O}^{\mathrm{v}}-\mathrm{O} 13-\mathrm{O} 2$ & $147.07(8)$ \\
\hline $\mathrm{O} 5-\mathrm{O} 13-\mathrm{O} 2$ & $92.32(7)$ \\
\hline $\mathrm{O} 8-\mathrm{O} 13-\mathrm{O} 2$ & $96.59(7)$ \\
\hline $\mathrm{Tm}-\mathrm{O} 13-\mathrm{O}^{3^{\mathrm{iv}}}$ & $113.42(8)$ \\
\hline $\mathrm{O}^{\mathrm{v}}-\mathrm{O} 13-\mathrm{O}^{\mathrm{iv}}$ & $108.73(8)$ \\
\hline $\mathrm{O} 5-\mathrm{O} 13-\mathrm{O}^{\mathrm{iv}}$ & $155.31(9)$ \\
\hline $\mathrm{O} 8-\mathrm{O} 13-\mathrm{O}^{\mathrm{iv}}$ & $62.20(7)$ \\
\hline $\mathrm{O} 2-\mathrm{O} 13-\mathrm{O}^{3}{ }^{\mathrm{iv}}$ & $101.59(7)$ \\
\hline
\end{tabular}




\begin{tabular}{|c|c|c|c|}
\hline $\mathrm{O} 6-\mathrm{O} 5-\mathrm{O} 12$ & $111.87(10)$ & $\mathrm{Tm}-\mathrm{O} 13-\mathrm{O} 10^{\mathrm{iii}}$ & $121.66(7)$ \\
\hline $\mathrm{Tm}-\mathrm{O} 5-\mathrm{O} 12$ & $50.68(5)$ & $\mathrm{O} 5^{\mathrm{v}}-\mathrm{O} 13-\mathrm{O} 10^{\mathrm{iii}}$ & $110.19(8)$ \\
\hline $\mathrm{O} 13 \mathrm{v}-\mathrm{O} 5-\mathrm{O} 12$ & $126.93(9)$ & $\mathrm{O} 5-\mathrm{O} 13-\mathrm{O} 10^{\mathrm{iii}}$ & $124.94(8)$ \\
\hline $\mathrm{O} 13-\mathrm{O} 5-\mathrm{O} 12$ & $103.34(7)$ & $\mathrm{O} 8-\mathrm{O} 13-\mathrm{O} 10^{\mathrm{iii}}$ & $132.34(8)$ \\
\hline $\mathrm{O} 7-\mathrm{O} 5-\mathrm{O} 12$ & $58.56(6)$ & $\mathrm{O} 2-\mathrm{O} 13-\mathrm{O} 10^{\mathrm{iii}}$ & $62.57(6)$ \\
\hline $\mathrm{N} 2-\mathrm{O} 5-\mathrm{O} 5^{\mathrm{v}}$ & $121.20(17)$ & $\mathrm{O} 3^{\mathrm{iv}}-\mathrm{O} 13-\mathrm{O} 10^{\mathrm{iii}}$ & $79.74(7)$ \\
\hline $\mathrm{O} 4-\mathrm{O} 5-\mathrm{O} 5^{\mathrm{v}}$ & $128.22(13)$ & $\mathrm{Tm}-\mathrm{O} 13-\mathrm{O} 1$ & $51.90(5)$ \\
\hline $\mathrm{O} 6-\mathrm{O} 5-\mathrm{O} 5^{\mathrm{v}}$ & $107.16(11)$ & $\mathrm{O} 5^{\mathrm{v}}-\mathrm{O} 13-\mathrm{O} 1$ & $163.64(9)$ \\
\hline $\mathrm{Tm}-\mathrm{O} 5-\mathrm{O} 5^{\mathrm{v}}$ & $107.25(8)$ & $\mathrm{O} 5-\mathrm{O} 13-\mathrm{O} 1$ & $108.19(7)$ \\
\hline $\mathrm{O} 13^{\mathrm{v}}-\mathrm{O} 5-\mathrm{O} 5^{\mathrm{v}}$ & $57.69(6)$ & $\mathrm{O} 8-\mathrm{O} 13-\mathrm{O} 1$ & $56.27(6)$ \\
\hline $\mathrm{O} 13-\mathrm{O} 5-\mathrm{O}^{\mathrm{v}}$ & $57.60(7)$ & $\mathrm{O} 2-\mathrm{O} 13-\mathrm{O} 1$ & $42.63(5)$ \\
\hline $\mathrm{O} 7-\mathrm{O} 5-\mathrm{O} 5^{\mathrm{v}}$ & $82.40(7)$ & $\mathrm{O} 3^{\mathrm{iv}}-\mathrm{O} 13-\mathrm{O} 1$ & $71.17(7)$ \\
\hline $\mathrm{O} 12-\mathrm{O} 5-\mathrm{O} 5^{\mathrm{v}}$ & $140.96(9)$ & 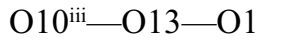 & $86.04(6)$ \\
\hline $\mathrm{N} 2-\mathrm{O} 6-\mathrm{O} 5$ & $29.68(12)$ & $\mathrm{Tm}-\mathrm{O} 13-\mathrm{O} 7$ & $48.90(5)$ \\
\hline $\mathrm{N} 2-\mathrm{O} 6-\mathrm{O} 4$ & $29.23(11)$ & $\mathrm{O}^{\mathrm{v}}-\mathrm{O} 13-\mathrm{O} 7$ & $79.82(7)$ \\
\hline $\mathrm{O} 5-\mathrm{O} 6-\mathrm{O} 4$ & $58.91(7)$ & $\mathrm{O} 5-\mathrm{O} 13-\mathrm{O} 7$ & $56.15(6)$ \\
\hline $\mathrm{N} 2-\mathrm{O} 6-\mathrm{O} 14^{\mathrm{vi}}$ & $99.61(16)$ & $\mathrm{O} 8-\mathrm{O} 13-\mathrm{O} 7$ & $41.95(5)$ \\
\hline $\mathrm{O} 5-\mathrm{O} 6-\mathrm{O} 14^{\mathrm{vi}}$ & $122.89(10)$ & $\mathrm{O} 2-\mathrm{O} 13-\mathrm{O} 7$ & $107.68(8)$ \\
\hline $\mathrm{O} 4-\mathrm{O} 6-\mathrm{O} 14^{\mathrm{vi}}$ & $75.58(9)$ & $\mathrm{O} 3^{\mathrm{iv}}-\mathrm{O} 13-\mathrm{O} 7$ & $99.84(7)$ \\
\hline $\mathrm{N} 2-\mathrm{O} 6-\mathrm{O} 9^{\mathrm{v}}$ & $136.91(18)$ & 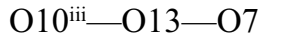 & $169.66(8)$ \\
\hline $\mathrm{O} 5-\mathrm{O} 6-\mathrm{O} 9^{\mathrm{v}}$ & $124.28(11)$ & $\mathrm{O} 1-\mathrm{O} 13-\mathrm{O} 7$ & $84.06(7)$ \\
\hline $\mathrm{O} 4-\mathrm{O} 6-\mathrm{O}^{\mathrm{v}}$ & $134.81(12)$ & $\mathrm{Tm}-\mathrm{O} 13-\mathrm{O}^{\mathrm{v}}$ & $111.12(7)$ \\
\hline $\mathrm{O} 14^{\mathrm{vi}}-\mathrm{O} 6-\mathrm{O} 9^{\mathrm{v}}$ & $67.17(7)$ & $05^{\mathrm{v}}-\mathrm{O} 13-\mathrm{O}^{\mathrm{v}}$ & $41.08(5)$ \\
\hline $\mathrm{N} 2-\mathrm{O} 6-\mathrm{O} 9^{\mathrm{vi}}$ & $142.10(16)$ & $\mathrm{O} 5-\mathrm{O} 13-\mathrm{O}^{\mathrm{v}}$ & $86.61(7)$ \\
\hline $\mathrm{O} 5-\mathrm{O} 6-\mathrm{O} 9^{\mathrm{vi}}$ & $169.33(12)$ & $\mathrm{O} 8-\mathrm{O} 13-\mathrm{O}^{\mathrm{v}}$ & $71.96(6)$ \\
\hline $\mathrm{O} 4-\mathrm{O} 6-\mathrm{O} 9^{\mathrm{vi}}$ & $113.34(10)$ & $\mathrm{O} 2-\mathrm{O} 13-\mathrm{O}^{\mathrm{v}}$ & $168.28(9)$ \\
\hline $\mathrm{O} 14^{\mathrm{vi}}-\mathrm{O} 6-\mathrm{O} 9^{\mathrm{vi}}$ & $57.38(6)$ & $\mathrm{O}^{\mathrm{iv}}-\mathrm{O} 13-\mathrm{O}^{\mathrm{v}}$ & $75.62(6)$ \\
\hline $\mathrm{O} 9^{\mathrm{v}}-\mathrm{O} 6-\mathrm{O} 9^{\mathrm{vi}}$ & $66.27(8)$ & $\mathrm{O} 10^{\mathrm{iii}}-\mathrm{O} 13-\mathrm{O}^{\mathrm{v}}$ & $127.02(8)$ \\
\hline $\mathrm{O} 9-\mathrm{N} 3-\mathrm{O} 8$ & $123.31(19)$ & $\mathrm{O} 1-\mathrm{O} 13-\mathrm{O}^{\mathrm{v}}$ & $126.97(7)$ \\
\hline $\mathrm{O} 9-\mathrm{N} 3-\mathrm{O} 7$ & $121.3(2)$ & $\mathrm{O} 7-\mathrm{O} 13-\mathrm{O}^{\mathrm{v}}$ & $62.22(6)$ \\
\hline $\mathrm{O} 8-\mathrm{N} 3-\mathrm{O} 7$ & $115.44(17)$ & $\mathrm{Tm}-\mathrm{O} 13-\mathrm{H} 7$ & $119(3)$ \\
\hline $\mathrm{O} 9-\mathrm{N} 3-\mathrm{Tm}$ & $173.32(18)$ & $\mathrm{O}^{\mathrm{v}}-\mathrm{O} 13-\mathrm{H} 7$ & $10(3)$ \\
\hline $\mathrm{O} 8-\mathrm{N} 3-\mathrm{Tm}$ & $61.80(10)$ & $\mathrm{O} 5-\mathrm{O} 13-\mathrm{H} 7$ & $64(3)$ \\
\hline $\mathrm{O} 7-\mathrm{N} 3-\mathrm{Tm}$ & $53.84(10)$ & $\mathrm{O} 8-\mathrm{O} 13-\mathrm{H} 7$ & $119(3)$ \\
\hline $\mathrm{N} 3-\mathrm{O} 7-\mathrm{O} 8$ & $31.79(10)$ & $\mathrm{O} 2-\mathrm{O} 13-\mathrm{H} 7$ & $138(3)$ \\
\hline $\mathrm{N} 3-\mathrm{O} 7-\mathrm{O} 9$ & $28.42(11)$ & $\mathrm{O}^{\mathrm{iv}}-\mathrm{O} 13-\mathrm{H} 7$ & $114(3)$ \\
\hline $\mathrm{O} 8-\mathrm{O} 7-\mathrm{O} 9$ & $60.21(8)$ & 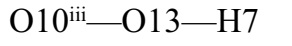 & $102(3)$ \\
\hline $\mathrm{N} 3-\mathrm{O} 7-\mathrm{Tm}$ & $100.41(12)$ & $\mathrm{O} 1-\mathrm{O} 13-\mathrm{H} 7$ & $171(3)$ \\
\hline $\mathrm{O} 8-\mathrm{O} 7-\mathrm{Tm}$ & $68.76(6)$ & $\mathrm{O} 7-\mathrm{O} 13-\mathrm{H} 7$ & $88(3)$ \\
\hline $\mathrm{O} 9-\mathrm{O} 7-\mathrm{Tm}$ & $128.67(10)$ & $\mathrm{O}^{\mathrm{v}}-\mathrm{O} 13-\mathrm{H} 7$ & $51(3)$ \\
\hline $\mathrm{N} 3-\mathrm{O} 7-\mathrm{O} 12^{\mathrm{vii}}$ & $109.38(13)$ & $\mathrm{Tm}-\mathrm{O} 13-\mathrm{H} 8$ & $119(3)$ \\
\hline $\mathrm{O} 8-\mathrm{O} 7-\mathrm{O} 12^{\mathrm{vii}}$ & $139.23(9)$ & $\mathrm{O} 5^{\mathrm{v}}-\mathrm{O} 13-\mathrm{H} 8$ & $97(3)$ \\
\hline $\mathrm{O} 9-\mathrm{O} 7-\mathrm{O} 12^{\mathrm{vii}}$ & $82.11(8)$ & $\mathrm{O} 5-\mathrm{O} 13-\mathrm{H} 8$ & $148(3)$ \\
\hline $\mathrm{Tm}-\mathrm{O} 7-\mathrm{O} 12^{\mathrm{vii}}$ & $148.08(8)$ & $\mathrm{O} 8-\mathrm{O} 13-\mathrm{H} 8$ & $63(3)$ \\
\hline $\mathrm{N} 3-\mathrm{O} 7-\mathrm{O} 12$ & $135.69(15)$ & $\mathrm{O} 2-\mathrm{O} 13-\mathrm{H} 8$ & $114(3)$ \\
\hline $\mathrm{O} 8-\mathrm{O} 7-\mathrm{O} 12$ & $112.27(9)$ & $\mathrm{O} 3^{\mathrm{iv}}-\mathrm{O} 13-\mathrm{H} 8$ & $12(3)$ \\
\hline $\mathrm{O} 9-\mathrm{O} 7-\mathrm{O} 12$ & $146.72(12)$ & $\mathrm{O} 10^{\mathrm{iii}-\mathrm{O} 13-\mathrm{H} 8}$ & $86(3)$ \\
\hline $\mathrm{Tm}-\mathrm{O} 7-\mathrm{O} 12$ & $52.72(5)$ & $\mathrm{O} 1-\mathrm{O} 13-\mathrm{H} 8$ & $82(3)$ \\
\hline
\end{tabular}




\begin{tabular}{|c|c|c|c|}
\hline $\mathrm{O} 12^{\mathrm{vii}}-\mathrm{O} 7-\mathrm{O} 12$ & $107.80(7)$ & $\mathrm{O} 7-\mathrm{O} 13-\mathrm{H} 8$ & $96(3)$ \\
\hline $\mathrm{N} 3-\mathrm{O} 7-\mathrm{O} 5$ & $138.14(16)$ & $\mathrm{O}^{\mathrm{v}}-\mathrm{O} 13-\mathrm{H} 8$ & $64(3)$ \\
\hline $\mathrm{O} 8-\mathrm{O} 7-\mathrm{O} 5$ & $112.81(9)$ & $\mathrm{H} 7-\mathrm{O} 13-\mathrm{H} 8$ & $103(4)$ \\
\hline $\mathrm{O} 9-\mathrm{O} 7-\mathrm{O} 5$ & $151.26(12)$ & $\mathrm{O} 12^{\mathrm{xi}}-\mathrm{O} 14-\mathrm{O} 11^{\mathrm{i}}$ & $99.62(8)$ \\
\hline $\mathrm{Tm}-\mathrm{O} 7-\mathrm{O} 5$ & $56.88(5)$ & $\mathrm{O} 12^{\mathrm{xi}}-\mathrm{O} 14-\mathrm{O} 8$ & $155.24(10)$ \\
\hline $\mathrm{O} 12^{\mathrm{vii}-\mathrm{O} 7-\mathrm{O} 5}$ & $92.35(7)$ & $\mathrm{O} 11^{\mathrm{i}}-\mathrm{O} 14-\mathrm{O} 8$ & $104.73(8)$ \\
\hline $\mathrm{O} 12-\mathrm{O} 7-\mathrm{O} 5$ & $61.71(6)$ & O12 $2^{\mathrm{xi}}-\mathrm{O} 14-\mathrm{O} 9$ & $124.30(10)$ \\
\hline $\mathrm{N} 3-\mathrm{O} 7-\mathrm{O} 10$ & $79.13(13)$ & $\mathrm{O} 11^{\mathrm{i}}-\mathrm{O} 14-\mathrm{O} 9$ & $123.04(11)$ \\
\hline $\mathrm{O} 8-\mathrm{O} 7-\mathrm{O} 10$ & $60.53(7)$ & $\mathrm{O} 8-\mathrm{O} 14-\mathrm{O} 9$ & $42.34(5)$ \\
\hline $\mathrm{O} 9-\mathrm{O} 7-\mathrm{O} 10$ & $97.66(9)$ & $\mathrm{O} 12^{\mathrm{xi}}-\mathrm{O} 14-\mathrm{O} 3^{\mathrm{iv}}$ & $108.74(10)$ \\
\hline $\mathrm{Tm}-\mathrm{O} 7-\mathrm{O} 10$ & $49.32(5)$ & $\mathrm{O} 11^{\mathrm{i}}-\mathrm{O} 14-\mathrm{O}^{\mathrm{iv}}$ & $109.99(9)$ \\
\hline $\mathrm{O} 12^{\mathrm{vii}}-\mathrm{O} 7-\mathrm{O} 10$ & $146.96(10)$ & $\mathrm{O} 8-\mathrm{O} 14-\mathrm{O}^{\mathrm{iv}}$ & $58.46(7)$ \\
\hline $\mathrm{O} 12-\mathrm{O} 7-\mathrm{O} 10$ & $56.57(6)$ & $\mathrm{O} 9-\mathrm{O} 14-\mathrm{O}^{\text {iv }}$ & $89.88(8)$ \\
\hline $\mathrm{O} 5-\mathrm{O} 7-\mathrm{O} 10$ & $101.99(7)$ & $\mathrm{O} 12^{\mathrm{xi}}-\mathrm{O} 14-\mathrm{O}^{\mathrm{viii}}$ & $75.69(8)$ \\
\hline $\mathrm{N} 3-\mathrm{O} 7-\mathrm{O} 13$ & 82.94 (13) & $\mathrm{O} 11^{\mathrm{i}}-\mathrm{O} 14-\mathrm{O}^{\mathrm{viii}}$ & $102.46(10)$ \\
\hline $\mathrm{O} 8-\mathrm{O} 7-\mathrm{O} 13$ & $59.99(7)$ & $\mathrm{O} 8-\mathrm{O} 14-\mathrm{O}^{\text {viii }}$ & $103.01(9)$ \\
\hline $\mathrm{O} 9-\mathrm{O} 7-\mathrm{O} 13$ & $104.46(10)$ & $\mathrm{O} 9-\mathrm{O} 14-\mathrm{O}^{\mathrm{viii}}$ & $62.42(7)$ \\
\hline $\mathrm{Tm}-\mathrm{O} 7-\mathrm{O} 13$ & $49.20(5)$ & $\mathrm{O} 3^{\text {iv }}-\mathrm{O} 14-\mathrm{O}^{\text {viii }}$ & $145.65(9)$ \\
\hline $\mathrm{O} 12^{\mathrm{vii}-\mathrm{O} 7-\mathrm{O} 13}$ & $122.65(9)$ & $\mathrm{O} 12^{\mathrm{xi}}-\mathrm{O} 14-\mathrm{H} 9$ & $114(3)$ \\
\hline $\mathrm{O} 12-\mathrm{O} 7-\mathrm{O} 13$ & $96.65(7)$ & 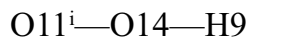 & $115(3)$ \\
\hline $\mathrm{O} 5-\mathrm{O} 7-\mathrm{O} 13$ & $55.37(6)$ & $\mathrm{O} 8-\mathrm{O} 14-\mathrm{H} 9$ & $50(3)$ \\
\hline $\mathrm{O} 10-\mathrm{O} 7-\mathrm{O} 13$ & $89.60(7)$ & $\mathrm{O} 9-\mathrm{O} 14-\mathrm{H} 9$ & $80(3)$ \\
\hline $\mathrm{N} 3-\mathrm{O} 8-\mathrm{O} 7$ & $32.77(10)$ & $\mathrm{O}^{\mathrm{iv}}-\mathrm{O} 14-\mathrm{H} 9$ & $10(3)$ \\
\hline $\mathrm{N} 3-\mathrm{O} 8-\mathrm{O} 9$ & $27.84(10)$ & $\mathrm{O}^{\text {viii_-O14-H9 }}$ & $137(3)$ \\
\hline $\mathrm{O} 7-\mathrm{O} 8-\mathrm{O} 9$ & $60.61(8)$ & $\mathrm{O} 12^{\mathrm{xi}}-\mathrm{O} 14-\mathrm{H} 10$ & $104(5)$ \\
\hline $\mathrm{N} 3-\mathrm{O} 8-\mathrm{Tm}$ & $92.77(11)$ & $\mathrm{O} 11^{\mathrm{i}}-\mathrm{O} 14-\mathrm{H} 10$ & $120(5)$ \\
\hline $\mathrm{O} 7-\mathrm{O} 8-\mathrm{Tm}$ & $60.13(6)$ & $\mathrm{O} 8-\mathrm{O} 14-\mathrm{H} 10$ & $67(4)$ \\
\hline $\mathrm{O} 9-\mathrm{O} 8-\mathrm{Tm}$ & $120.49(8)$ & $\mathrm{O} 9-\mathrm{O} 14-\mathrm{H} 10$ & $25(4)$ \\
\hline $\mathrm{N} 3-\mathrm{O} 8-\mathrm{O} 10$ & $93.66(14)$ & $\mathrm{O}^{\mathrm{iv}}-\mathrm{O} 14-\mathrm{H} 10$ & $112(5)$ \\
\hline $\mathrm{O} 7-\mathrm{O} 8-\mathrm{O} 10$ & $76.28(8)$ & 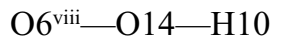 & $37(4)$ \\
\hline $\mathrm{O} 9-\mathrm{O} 8-\mathrm{O} 10$ & $107.87(10)$ & $\mathrm{H} 9-\mathrm{O} 14-\mathrm{H} 10$ & $103(5)$ \\
\hline $\mathrm{Tm}-\mathrm{O} 8-\mathrm{O} 10$ & $52.43(5)$ & & \\
\hline
\end{tabular}

Symmetry codes: (i) $-x,-y+1,-z+1$; (ii) $-x+1,-y,-z+1$; (iii) $x+1, y, z$; (iv) $-x+1,-y+1,-z+1$; (v) $-x+1,-y+1,-z$; (vi) $x+1, y-1, z$; (vii) $-x,-y+1,-z$; (viii) $x-1, y+1, z$; (ix) $x-1, y, z$; (x) $x, y-1, z$; (xi) $x, y+1, z$.

Hydrogen-bond geometry $\left(\AA,{ }^{\circ}\right)$

\begin{tabular}{lllll}
\hline$D-\mathrm{H} \cdots A$ & $D-\mathrm{H}$ & $\mathrm{H} \cdots A$ & $D \cdots A$ & $D-\mathrm{H} \cdots A$ \\
\hline $\mathrm{O} 10-\mathrm{H} 1 \cdots \mathrm{O} 1^{\mathrm{i}}$ & $0.82(4)$ & $2.03(4)$ & $2.849(2)$ & $174(3)$ \\
$\mathrm{O} 10-\mathrm{H} 2 \cdots \mathrm{O} 13^{\text {ix }}$ & $0.78(4)$ & $2.32(4)$ & $2.996(3)$ & $145(3)$ \\
$\mathrm{O} 10-\mathrm{H} 2 \cdots \mathrm{O} 2^{\text {ix }}$ & $0.78(4)$ & $2.48(4)$ & $3.035(3)$ & $129(3)$ \\
$\mathrm{O} 11-\mathrm{H} 3 \cdots \mathrm{O} 14^{\mathrm{i}}$ & $0.77(5)$ & $1.98(5)$ & $2.739(3)$ & $167(5)$ \\
$\mathrm{O} 11-\mathrm{H} 4 \cdots \mathrm{O} 2^{\mathrm{ii}}$ & $0.85(4)$ & $2.03(4)$ & $2.874(2)$ & $171(4)$ \\
$\mathrm{O} 12-\mathrm{H} 5 \cdots \mathrm{O} 14^{\mathrm{x}}$ & $0.83(4)$ & $1.90(4)$ & $2.715(3)$ & $165(4)$ \\
$\mathrm{O} 12-\mathrm{H} 6 \cdots \mathrm{O} 7^{\text {vii }}$ & $0.83(4)$ & $1.95(4)$ & $2.776(2)$ & $174(4)$ \\
$\mathrm{O} 13-\mathrm{H} 7 \cdots \mathrm{O} 5^{\mathrm{v}}$ & $0.82(5)$ & $1.98(5)$ & $2.784(2)$ & $166(4)$ \\
$\mathrm{O} 13-\mathrm{H} 8 \cdots \mathrm{O} 3^{\text {iv }}$ & $0.86(4)$ & $2.12(4)$ & $2.953(3)$ & $163(4)$ \\
$\mathrm{O} 14-\mathrm{H} 9 \cdots \mathrm{O} 3^{\text {iv }}$ & $0.84(4)$ & $2.27(5)$ & $3.095(3)$ & $167(4)$
\end{tabular}




\begin{tabular}{lllll}
$\mathrm{O} 14-\mathrm{H} 10 \cdots \mathrm{O} 9$ & $0.68(6)$ & $2.44(6)$ & $3.040(3)$ & $148(5)$ \\
$\mathrm{O} 14-\mathrm{H} 10 \cdots \mathrm{O}^{\text {viii }}$ & $0.68(6)$ & $2.62(6)$ & $3.132(4)$ & $134(5)$ \\
\hline
\end{tabular}

Symmetry codes: (i) $-x,-y+1,-z+1$; (ii) $-x+1,-y,-z+1$; (iv) $-x+1,-y+1,-z+1$; (v) $-x+1,-y+1,-z$; (vii) $-x,-y+1,-z$; (viii) $x-1, y+1, z$; (ix) $x-1, y, z$; (x) $x, y-1, z$.

Thulium nitrate (TONii-3_223K)

Crystal data

$\left[\mathrm{Tm}\left(\mathrm{NO}_{3}\right)_{3}\left(\mathrm{H}_{2} \mathrm{O}\right)_{4}\right] \cdot 2 \mathrm{H}_{2} \mathrm{O}$

$M_{r}=463.06$

Triclinic, $P \overline{1}$

$a=6.7050(3) \AA$

$b=8.9733(4) \AA$

$c=11.4915(6) \AA$

$\alpha=70.924(4)^{\circ}$

$\beta=88.908(4)^{\circ}$

$\gamma=68.923(4)^{\circ}$

$V=605.90(5) \AA^{3}$

\section{Data collection}

STOE StadiVari diffractometer

Radiation source: Genix 3D HF Mo

Graded multilayer mirror monochromator

Detector resolution: 5.81 pixels $\mathrm{mm}^{-1}$

$\omega$ scans

Absorption correction: empirical (using intensity measurements)

(X-AREA; Stoe, 2015)

\section{Refinement}

Refinement on $F^{2}$

Least-squares matrix: full

$R\left[F^{2}>2 \sigma\left(F^{2}\right)\right]=0.016$

$w R\left(F^{2}\right)=0.035$

$S=0.91$

4385 reflections

221 parameters

0 restraints

Primary atom site location: structure-invariant direct methods

Secondary atom site location: difference Fourier map

\section{Special details}

Geometry. All esds (except the esd in the dihedral angle between two 1.s. planes) are estimated using the full covariance matrix. The cell esds are taken into account individually in the estimation of esds in distances, angles and torsion angles; correlations between esds in cell parameters are only used when they are defined by crystal symmetry. An approximate (isotropic) treatment of cell esds is used for estimating esds involving l.s. planes.

Fractional atomic coordinates and isotropic or equivalent isotropic displacement parameters $\left(\AA^{2}\right)$

\begin{tabular}{lllll}
\hline & $x$ & $y$ & $z$ & $U_{\mathrm{iso}} * / U_{\text {eq }}$ \\
\hline $\operatorname{Tm}$ & $0.19543(2)$ & $0.41275(2)$ & $0.27232(2)$ & $0.01566(4)$ \\
\hline
\end{tabular}

$Z=2$

$F(000)=444$

$D_{\mathrm{x}}=2.538 \mathrm{Mg} \mathrm{m}^{-3}$

Mo $K \alpha$ radiation, $\lambda=0.71073 \AA$

Cell parameters from 47483 reflections

$\mu=7.41 \mathrm{~mm}^{-1}$

$0.4 \times 0.2 \times 0.15 \mathrm{~mm}$

$T_{\min }=0.615, T_{\max }=1.000$

29880 measured reflections

4385 independent reflections

3899 reflections with $I>2 \sigma(I)$

$R_{\text {int }}=0.031$

$\theta_{\text {max }}=32.5^{\circ}, \theta_{\min }=3.3^{\circ}$

$h=-10 \rightarrow 10$

$k=-13 \rightarrow 13$

$l=-17 \rightarrow 17$

Hydrogen site location: difference Fourier map

All $\mathrm{H}$-atom parameters refined

$w=1 /\left[\sigma^{2}\left(F_{\mathrm{o}}^{2}\right)+(0.020 P)^{2}\right]$

where $P=\left(F_{\mathrm{o}}^{2}+2 F_{\mathrm{c}}^{2}\right) / 3$

$(\Delta / \sigma)_{\max }<0.001$

$\Delta \rho_{\max }=1.11$ e $\AA^{-3}$

$\Delta \rho_{\min }=-1.18$ e $\AA^{-3}$

Extinction correction: SHELXL-2014/7

(Sheldrick, 2015),

$\mathrm{Fc}^{*}=\mathrm{kFc}\left[1+0.001 \mathrm{xFc}^{2} \lambda^{3} / \sin (2 \theta)\right]^{-1 / 4}$

Extinction coefficient: 0.0687 (8) $\theta=2.6-36.6^{\circ}$ 


$\begin{array}{lllll}\text { N1 } & -0.1763(3) & 0.7245(2) & 0.18627(18) & 0.0219(3) \\ \text { O1 } & -0.1088(3) & 0.6386(2) & 0.29939(16) & 0.0306(4) \\ \text { O2 } & -0.0716(3) & 0.6650(2) & 0.10973(17) & 0.0332(4) \\ \text { O3 } & -0.3348(3) & 0.8577(2) & 0.15483(19) & 0.0338(4) \\ \text { N2 } & 0.0790(3) & 0.1833(2) & 0.18432(18) & 0.0239(4) \\ \text { O4 } & 0.2565(3) & 0.1444(2) & 0.24492(17) & 0.0283(3) \\ \text { O5 } & -0.0588(3) & 0.3320(2) & 0.16876(17) & 0.0282(3) \\ \text { O6 } & 0.0422(4) & 0.0843(2) & 0.14426(19) & 0.0358(4) \\ \text { N3 } & 0.0931(3) & 0.1599(2) & 0.50588(18) & 0.0216(3) \\ \text { O7 } & -0.0255(3) & 0.2987(2) & 0.42350(16) & 0.0267(3) \\ \text { O8 } & 0.2889(3) & 0.1045(3) & 0.4993(2) & 0.0356(4) \\ \text { O9 } & 0.0120(3) & 0.0838(2) & 0.59033(18) & 0.0367(4) \\ \text { O10 } & 0.3559(3) & 0.6083(2) & 0.22157(17) & 0.0282(4) \\ \text { H1 } & 0.361(6) & 0.677(5) & 0.151(4) & 0.049(10)^{*} \\ \text { H2 } & 0.385(7) & 0.637(6) & 0.269(4) & 0.051(11)^{*} \\ \text { O11 } & 0.2745(3) & 0.4539(2) & 0.45290(15) & 0.0234(3) \\ \text { H3 } & 0.200(7) & 0.521(5) & 0.484(4) & 0.048(11)^{*} \\ \text { H4 } & 0.367(6) & 0.390(5) & 0.499(3) & 0.034(9)^{*} \\ \text { O12 } & 0.5603(3) & 0.2458(2) & 0.32343(18) & 0.0251(3) \\ \text { H5 } & 0.606(6) & 0.147(5) & 0.366(3) & 0.037(9)^{*} \\ \text { H6 } & 0.652(7) & 0.283(5) & 0.310(4) & 0.049(11)^{*} \\ \text { O13 } & 0.3038(3) & 0.4359(2) & 0.07487(15) & 0.0230(3) \\ \text { H7 } & 0.400(7) & 0.363(5) & 0.063(4) & 0.046(10)^{*} \\ \text { H8 } & 0.236(7) & 0.500(6) & 0.006(4) & 0.059(12)^{*} \\ \text { O14 } & 0.3663(3) & 0.8009(2) & -0.01622(17) & 0.0272(3) \\ \text { H9 } & 0.252(7) & 0.832(5) & -0.055(4) & 0.039(10)^{*} \\ \text { H10 } & 0.391(7) & 0.883(6) & -0.019(4) & 0.058(12)^{*} \\ \text { O15 } & 0.4146(3) & 0.7754(2) & 0.36863(17) & 0.0244(3) \\ \text { H11 } & 0.301(7) & 0.813(6) & 0.390(4) & 0.052(12)^{*} \\ \text { H12 } & 0.439(8) & 0.856(6) & 0.327(4) & 0.066(14)^{*} \\ & & & & \end{array}$

Atomic displacement parameters $\left(\AA^{2}\right)$

\begin{tabular}{lllllll}
\hline & $U^{11}$ & $U^{22}$ & $U^{33}$ & $U^{12}$ & $U^{13}$ & $U^{23}$ \\
\hline Tm & $0.01512(5)$ & $0.01444(4)$ & $0.01475(5)$ & $-0.00368(3)$ & $0.00155(2)$ & $-0.00370(3)$ \\
N1 & $0.0208(8)$ & $0.0134(7)$ & $0.0251(9)$ & $-0.0038(6)$ & $0.0013(7)$ & $-0.0012(6)$ \\
O1 & $0.0303(9)$ & $0.0238(8)$ & $0.0203(8)$ & $0.0029(7)$ & $0.0054(6)$ & $-0.0003(6)$ \\
O2 & $0.0341(9)$ & $0.0324(9)$ & $0.0227(8)$ & $0.0000(7)$ & $0.0010(7)$ & $-0.0103(7)$ \\
O3 & $0.0260(8)$ & $0.0168(7)$ & $0.0389(10)$ & $0.0031(6)$ & $0.0023(7)$ & $0.0028(7)$ \\
N2 & $0.0287(9)$ & $0.0200(8)$ & $0.0211(8)$ & $-0.0114(7)$ & $-0.0005(7)$ & $-0.0019(7)$ \\
O4 & $0.0266(8)$ & $0.0189(7)$ & $0.0328(9)$ & $-0.0058(6)$ & $-0.0084(7)$ & $-0.0028(6)$ \\
O5 & $0.0235(8)$ & $0.0266(8)$ & $0.0298(8)$ & $-0.0052(6)$ & $-0.0022(6)$ & $-0.0081(7)$ \\
O6 & $0.0485(12)$ & $0.0275(9)$ & $0.0345(10)$ & $-0.0192(8)$ & $-0.0063(8)$ & $-0.0084(7)$ \\
N3 & $0.0214(8)$ & $0.0162(7)$ & $0.0242(9)$ & $-0.0049(6)$ & $0.0059(7)$ & $-0.0056(6)$ \\
O7 & $0.0282(8)$ & $0.0178(7)$ & $0.0252(8)$ & $-0.0058(6)$ & $0.0035(6)$ & $0.0004(6)$ \\
O8 & $0.0191(8)$ & $0.0347(10)$ & $0.0532(12)$ & $-0.0060(7)$ & $0.0103(8)$ & $-0.0200(9)$ \\
O9 & $0.0394(10)$ & $0.0256(8)$ & $0.0320(9)$ & $-0.0102(8)$ & $0.0145(8)$ & $0.0039(7)$ \\
O10 & $0.0495(11)$ & $0.0276(8)$ & $0.0170(7)$ & $-0.0257(8)$ & $0.0066(7)$ & $-0.0073(6)$
\end{tabular}




\begin{tabular}{lllllll} 
O11 & $0.0259(8)$ & $0.0216(7)$ & $0.0160(7)$ & $-0.0025(6)$ & $-0.0007(6)$ & $-0.0050(6)$ \\
O12 & $0.0169(7)$ & $0.0180(7)$ & $0.0331(9)$ & $-0.0032(6)$ & $-0.0001(6)$ & $-0.0029(6)$ \\
O13 & $0.0257(8)$ & $0.0220(7)$ & $0.0165(7)$ & $-0.0040(6)$ & $0.0027(6)$ & $-0.0061(6)$ \\
O14 & $0.0336(9)$ & $0.0208(8)$ & $0.0258(8)$ & $-0.0112(7)$ & $0.0027(7)$ & $-0.0049(6)$ \\
O15 & $0.0267(8)$ & $0.0183(7)$ & $0.0249(8)$ & $-0.0065(6)$ & $-0.0009(7)$ & $-0.0050(6)$ \\
\hline
\end{tabular}

Geometric parameters $\left(A,{ }^{o}\right)$

\begin{tabular}{|c|c|c|c|}
\hline $\mathrm{Tm}-\mathrm{O} 10$ & $2.2897(18)$ & $\mathrm{O} 7-\mathrm{O} 8$ & $2.162(3)$ \\
\hline $\mathrm{Tm}-\mathrm{O} 11$ & $2.3255(17)$ & $\mathrm{O} 7-\mathrm{O} 9$ & $2.171(2)$ \\
\hline $\mathrm{Tm}-\mathrm{O} 12$ & $2.3276(17)$ & $\mathrm{O} 7-\mathrm{O} 11$ & $2.907(3)$ \\
\hline $\mathrm{Tm}-\mathrm{O} 13$ & $2.3360(16)$ & $\mathrm{O} 7-\mathrm{O} 11^{\mathrm{i}}$ & $3.006(2)$ \\
\hline $\mathrm{Tm}-\mathrm{O} 1$ & $2.4039(17)$ & $\mathrm{O} 8-\mathrm{O} 9$ & $2.151(3)$ \\
\hline $\mathrm{Tm}-\mathrm{O} 4$ & $2.4179(18)$ & $\mathrm{O} 8-\mathrm{O}^{\mathrm{vii}}$ & $2.774(4)$ \\
\hline $\mathrm{Tm}-\mathrm{O} 7$ & $2.4677(17)$ & $\mathrm{O} 8-\mathrm{O} 12^{\mathrm{vii}}$ & $2.943(3)$ \\
\hline $\mathrm{Tm}-\mathrm{O} 2$ & $2.5034(18)$ & $\mathrm{O} 8-\mathrm{O} 11$ & $2.969(3)$ \\
\hline $\mathrm{Tm}-\mathrm{O} 5$ & $2.5252(18)$ & $\mathrm{O} 8-\mathrm{O} 12$ & $2.974(3)$ \\
\hline $\mathrm{Tm}-\mathrm{N} 1$ & $2.8787(18)$ & $\mathrm{O} 8-\mathrm{O} 15^{\text {viii }}$ & $3.184(3)$ \\
\hline $\mathrm{Tm}-\mathrm{N} 2$ & $2.902(2)$ & $\mathrm{O} 9-\mathrm{O} 15^{\mathrm{i}}$ & $2.782(3)$ \\
\hline $\mathrm{Tm}-\mathrm{O} 8$ & $2.991(2)$ & $\mathrm{O} 9-09^{\mathrm{vi}}$ & $2.970(5)$ \\
\hline $\mathrm{Tm}-\mathrm{N} 3$ & $3.1452(18)$ & $\mathrm{O} 9-\mathrm{O}^{\mathrm{vi}}$ & 3.009 (3) \\
\hline $\mathrm{N} 1-\mathrm{O} 3$ & $1.227(2)$ & $\mathrm{O} 10-\mathrm{O} 11$ & $2.714(2)$ \\
\hline $\mathrm{N} 1-\mathrm{O} 2$ & $1.251(3)$ & $\mathrm{O} 10-\mathrm{O} 15$ & $2.714(3)$ \\
\hline $\mathrm{N} 1-\mathrm{O} 1$ & $1.267(2)$ & $\mathrm{O} 10-\mathrm{O} 14$ & $2.730(3)$ \\
\hline $\mathrm{O} 1-\mathrm{O} 2$ & $2.134(2)$ & $\mathrm{O} 10-\mathrm{O} 13$ & $2.734(2)$ \\
\hline $\mathrm{O} 1-\mathrm{O} 3$ & $2.177(2)$ & $\mathrm{O} 10-\mathrm{O} 12$ & $2.863(3)$ \\
\hline $\mathrm{O} 1-\mathrm{O} 7$ & $2.758(2)$ & $\mathrm{O} 10-\mathrm{H} 1$ & $0.85(4)$ \\
\hline $\mathrm{O} 1-\mathrm{O} 11$ & $2.768(3)$ & $\mathrm{O} 10-\mathrm{H} 2$ & $0.73(5)$ \\
\hline $\mathrm{O} 1-\mathrm{O} 11^{\mathrm{i}}$ & $3.004(2)$ & $\mathrm{O} 11-\mathrm{O} 15^{\text {viii }}$ & $2.666(2)$ \\
\hline $\mathrm{O} 1-\mathrm{O} 10$ & $3.166(3)$ & $\mathrm{O} 11-\mathrm{O} 12$ & $2.917(3)$ \\
\hline $\mathrm{O} 1-\mathrm{O} 15^{\mathrm{ii}}$ & $3.174(3)$ & $\mathrm{O} 11-\mathrm{O}^{\mathrm{i}}$ & $3.004(2)$ \\
\hline $\mathrm{O} 2-\mathrm{O} 3$ & $2.175(3)$ & $\mathrm{O} 11-\mathrm{O}^{\mathrm{i}}$ & $3.006(2)$ \\
\hline $\mathrm{O} 2-\mathrm{O} 13$ & $2.738(3)$ & $\mathrm{O} 11-\mathrm{O} 15$ & 3.199 (3) \\
\hline $\mathrm{O} 2-\mathrm{O} 5$ & $2.809(3)$ & $\mathrm{O} 11-\mathrm{H} 3$ & $0.81(4)$ \\
\hline $\mathrm{O} 2-\mathrm{O} 10$ & $2.965(3)$ & $\mathrm{O} 11-\mathrm{H} 4$ & $0.74(4)$ \\
\hline $\mathrm{O} 2-\mathrm{O}^{\mathrm{iii}}$ & $3.102(3)$ & $\mathrm{O} 12-\mathrm{O} 8^{\mathrm{vii}}$ & $2.943(3)$ \\
\hline $\mathrm{O} 2-\mathrm{O} 13^{\mathrm{iii}}$ & $3.184(3)$ & $\mathrm{O} 12-\mathrm{O} 13$ & $2.986(3)$ \\
\hline $\mathrm{O} 3-\mathrm{O} 14^{\mathrm{iv}}$ & $2.885(2)$ & $\mathrm{O} 12-\mathrm{O} 9^{\mathrm{vii}}$ & $3.170(3)$ \\
\hline $\mathrm{O} 3-\mathrm{O} 15^{\mathrm{ii}}$ & $2.992(3)$ & $\mathrm{O} 12-\mathrm{H} 5$ & $0.80(4)$ \\
\hline $\mathrm{O} 3-\mathrm{O} 14^{\mathrm{ii}}$ & $3.111(3)$ & $\mathrm{O} 12-\mathrm{H} 6$ & $0.79(4)$ \\
\hline $\mathrm{N} 2-\mathrm{O} 6$ & $1.221(3)$ & $\mathrm{O} 13-014^{\mathrm{ix}}$ & $2.713(3)$ \\
\hline $\mathrm{N} 2-\mathrm{O} 4$ & $1.263(3)$ & $\mathrm{O} 13-\mathrm{O} 5^{\mathrm{iii}}$ & $2.963(2)$ \\
\hline $\mathrm{N} 2-\mathrm{O} 5$ & $1.277(3)$ & $\mathrm{O} 13-\mathrm{O} 2^{\mathrm{iii}}$ & $3.184(3)$ \\
\hline $\mathrm{O} 4-\mathrm{O} 5$ & $2.148(2)$ & $\mathrm{O} 13-\mathrm{H} 7$ & $0.78(4)$ \\
\hline $\mathrm{O} 4-\mathrm{O} 6$ & $2.173(3)$ & $\mathrm{O} 13-\mathrm{H} 8$ & $0.83(5)$ \\
\hline $\mathrm{O} 4-\mathrm{O} 12$ & $2.781(3)$ & $\mathrm{O} 14-013^{\mathrm{ix}}$ & $2.713(3)$ \\
\hline $\mathrm{O} 4-\mathrm{O} 13$ & $2.827(2)$ & $\mathrm{O} 14-\mathrm{O}^{\mathrm{iii}}$ & $2.804(3)$ \\
\hline $\mathrm{O} 4-\mathrm{O} 8$ & $2.829(3)$ & $\mathrm{O} 14-\mathrm{O}^{3 \mathrm{iv}}$ & $2.885(2)$ \\
\hline
\end{tabular}




\begin{tabular}{|c|c|c|c|}
\hline $\mathrm{O} 4-\mathrm{O} 15^{\mathrm{v}}$ & $2.926(2)$ & $\mathrm{O} 14-03^{\mathrm{x}}$ & $3.111(3)$ \\
\hline $\mathrm{O} 4-\mathrm{O} 7$ & $3.087(3)$ & $\mathrm{O} 14-\mathrm{H} 9$ & $0.80(4)$ \\
\hline $\mathrm{O} 5-\mathrm{O} 6$ & $2.190(3)$ & $\mathrm{O} 14-\mathrm{H} 10$ & $0.80(5)$ \\
\hline $\mathrm{O} 5-\mathrm{O} 7$ & $2.849(3)$ & $\mathrm{O} 15-\mathrm{O} 11^{\mathrm{vii}}$ & $2.666(2)$ \\
\hline $\mathrm{O} 5-\mathrm{O} 13^{\mathrm{iii}}$ & $2.963(2)$ & $\mathrm{O} 15-09^{\mathrm{i}}$ & $2.782(3)$ \\
\hline $\mathrm{O} 5-\mathrm{O} 13$ & $2.969(2)$ & $\mathrm{O} 15-\mathrm{O} 4^{\mathrm{xi}}$ & $2.926(2)$ \\
\hline $\mathrm{O} 6-\mathrm{O} 14^{\mathrm{iii}}$ & $2.804(3)$ & $\mathrm{O} 15-\mathrm{O}^{\mathrm{x}}$ & $2.992(3)$ \\
\hline $\mathrm{O} 6-\mathrm{O} 9^{\mathrm{vi}}$ & 3.009 (3) & $\mathrm{O} 15-\mathrm{O} 1^{\mathrm{x}}$ & $3.174(3)$ \\
\hline $\mathrm{O} 6-\mathrm{O} 2^{\mathrm{iii}}$ & $3.102(3)$ & $\mathrm{O} 15-\mathrm{O} 8^{\text {viii }}$ & $3.184(3)$ \\
\hline N3-O9 & $1.232(2)$ & O15-H11 & $0.79(5)$ \\
\hline $\mathrm{N} 3-\mathrm{O} 8$ & $1.236(3)$ & $\mathrm{O} 15-\mathrm{H} 12$ & $0.79(5)$ \\
\hline $\mathrm{N} 3-\mathrm{O} 7$ & $1.275(2)$ & & \\
\hline $\mathrm{O} 10-\mathrm{Tm}-\mathrm{O} 11$ & $72.03(6)$ & $\mathrm{O} 7-\mathrm{O} 8-\mathrm{O} 12$ & $100.29(9)$ \\
\hline $\mathrm{O} 10-\mathrm{Tm}-\mathrm{O} 12$ & $76.62(7)$ & $\mathrm{O} 8^{\mathrm{vii}}-\mathrm{O} 8-\mathrm{O} 12$ & $61.49(8)$ \\
\hline $\mathrm{O} 11-\mathrm{Tm}-\mathrm{O} 12$ & $77.63(7)$ & $\mathrm{O} 4-\mathrm{O} 8-\mathrm{O} 12$ & $57.20(6)$ \\
\hline $\mathrm{O} 10-\mathrm{Tm}-\mathrm{O} 13$ & $72.46(6)$ & $\mathrm{O} 12^{\mathrm{vii}}-\mathrm{O} 8-\mathrm{O} 12$ & $124.10(7)$ \\
\hline $\mathrm{O} 11-\mathrm{Tm}-\mathrm{O} 13$ & $141.28(6)$ & $\mathrm{O} 11-\mathrm{O} 8-\mathrm{O} 12$ & $58.78(6)$ \\
\hline $\mathrm{O} 12-\mathrm{Tm}-\mathrm{O} 13$ & $79.64(7)$ & $\mathrm{N} 3-\mathrm{O} 8-\mathrm{Tm}$ & $85.50(14)$ \\
\hline $\mathrm{O} 10-\mathrm{Tm}-\mathrm{O} 1$ & $84.81(7)$ & $\mathrm{O} 9-\mathrm{O} 8-\mathrm{Tm}$ & $114.83(9)$ \\
\hline $\mathrm{O} 11-\mathrm{Tm}-\mathrm{O} 1$ & $71.62(6)$ & $\mathrm{O} 7-\mathrm{O} 8-\mathrm{Tm}$ & $54.39(6)$ \\
\hline $\mathrm{O} 12-\mathrm{Tm}-\mathrm{O} 1$ & $147.76(7)$ & $\mathrm{O} 8^{\mathrm{vii}}-\mathrm{O} 8-\mathrm{Tm}$ & $106.06(10)$ \\
\hline $\mathrm{O} 13-\mathrm{Tm}-\mathrm{O} 1$ & $119.66(6)$ & $\mathrm{O} 4-\mathrm{O} 8-\mathrm{Tm}$ & $49.00(5)$ \\
\hline $\mathrm{O} 10-\mathrm{Tm}-\mathrm{O} 4$ & $136.37(7)$ & $\mathrm{O} 12^{\mathrm{vii}}-\mathrm{O} 8-\mathrm{Tm}$ & $164.48(8)$ \\
\hline $\mathrm{O} 11-\mathrm{Tm}-\mathrm{O} 4$ & $127.07(6)$ & $\mathrm{O} 11-\mathrm{O} 8-\mathrm{Tm}$ & $45.93(4)$ \\
\hline $\mathrm{O} 12-\mathrm{Tm}-\mathrm{O} 4$ & $71.71(6)$ & $\mathrm{O} 12-\mathrm{O} 8-\mathrm{Tm}$ & $45.93(5)$ \\
\hline $\mathrm{O} 13-\mathrm{Tm}-\mathrm{O} 4$ & $72.95(6)$ & $\mathrm{N} 3-\mathrm{O} 8-\mathrm{O} 15^{\text {viii }}$ & $118.44(15)$ \\
\hline $\mathrm{O} 1-\mathrm{Tm}-\mathrm{O} 4$ & $136.08(7)$ & $\mathrm{O} 9-\mathrm{O} 8-\mathrm{O} 15^{\text {viii }}$ & $114.99(11)$ \\
\hline $\mathrm{O} 10-\mathrm{Tm}-\mathrm{O} 7$ & $142.62(6)$ & $\mathrm{O} 7-\mathrm{O} 8-\mathrm{O} 15^{\text {viii }}$ & $113.47(9)$ \\
\hline $\mathrm{O} 11-\mathrm{Tm}-\mathrm{O} 7$ & $74.59(6)$ & $\mathrm{O} 8^{\mathrm{vii}}-\mathrm{O} 8-\mathrm{O} 15^{\mathrm{viii}}$ & $73.26(9)$ \\
\hline $\mathrm{O} 12-\mathrm{Tm}-\mathrm{O} 7$ & $112.05(6)$ & $\mathrm{O} 4-\mathrm{O} 8-\mathrm{O} 15^{\mathrm{viii}}$ & $125.89(8)$ \\
\hline $\mathrm{O} 13-\mathrm{Tm}-\mathrm{O} 7$ & $143.65(6)$ & $\mathrm{O} 12^{\mathrm{vii}}-\mathrm{O} 8-\mathrm{O} 15^{\mathrm{viii}}$ & $95.36(8)$ \\
\hline $\mathrm{O} 1-\mathrm{Tm}-\mathrm{O} 7$ & $68.96(6)$ & $\mathrm{O} 11-\mathrm{O} 8-\mathrm{O} 15^{\mathrm{viii}}$ & $51.20(6)$ \\
\hline $\mathrm{O} 4-\mathrm{Tm}-\mathrm{O} 7$ & $78.37(6)$ & $\mathrm{O} 12-\mathrm{O} 8-\mathrm{O} 15^{\text {viii }}$ & $68.83(7)$ \\
\hline $\mathrm{O} 10-\mathrm{Tm}-\mathrm{O} 2$ & $76.28(7)$ & $\mathrm{Tm}-\mathrm{O} 8-\mathrm{O} 15^{\mathrm{viii}}$ & $90.93(6)$ \\
\hline $\mathrm{O} 11-\mathrm{Tm}-\mathrm{O} 2$ & $116.27(6)$ & $\mathrm{N} 3-\mathrm{O} 9-\mathrm{O} 8$ & $29.45(12)$ \\
\hline $\mathrm{O} 12-\mathrm{Tm}-\mathrm{O} 2$ & $143.26(7)$ & $\mathrm{N} 3-\mathrm{O} 9-\mathrm{O} 7$ & $30.60(11)$ \\
\hline $\mathrm{O} 13-\mathrm{Tm}-\mathrm{O} 2$ & $68.82(6)$ & $\mathrm{O} 8-\mathrm{O} 9-\mathrm{O} 7$ & $60.05(9)$ \\
\hline $\mathrm{O} 1-\mathrm{Tm}-\mathrm{O} 2$ & $51.50(6)$ & $\mathrm{N} 3-\mathrm{O} 9-\mathrm{O} 15^{\mathrm{i}}$ & $122.54(15)$ \\
\hline $\mathrm{O} 4-\mathrm{Tm}-\mathrm{O} 2$ & $114.16(6)$ & $\mathrm{O} 8-\mathrm{O} 9-\mathrm{O} 15^{\mathrm{i}}$ & $151.29(11)$ \\
\hline $\mathrm{O} 7-\mathrm{Tm}-\mathrm{O} 2$ & $104.56(6)$ & $\mathrm{O} 7-\mathrm{O} 9-\mathrm{O} 15^{\mathrm{i}}$ & $92.26(9)$ \\
\hline $\mathrm{O} 10-\mathrm{Tm}-\mathrm{O} 5$ & $138.32(6)$ & $\mathrm{N} 3-\mathrm{O} 9-\mathrm{O} 9^{\mathrm{vi}}$ & $81.16(15)$ \\
\hline $\mathrm{O} 11-\mathrm{Tm}-\mathrm{O} 5$ & $143.50(6)$ & $\mathrm{O} 8-\mathrm{O} 9-\mathrm{O}^{\mathrm{vi}}$ & $81.92(10)$ \\
\hline $\mathrm{O} 12-\mathrm{Tm}-\mathrm{O} 5$ & $122.22(6)$ & $\mathrm{O} 7-\mathrm{O} 9-\mathrm{O} 9^{\mathrm{vi}}$ & $82.92(10)$ \\
\hline $\mathrm{O} 13-\mathrm{Tm}-\mathrm{O} 5$ & $75.17(6)$ & $\mathrm{O} 15^{\mathrm{i}}-\mathrm{O} 9-\mathrm{O} 9^{\mathrm{vi}}$ & $103.45(11)$ \\
\hline $\mathrm{O} 1-\mathrm{Tm}-\mathrm{O} 5$ & $89.05(6)$ & $\mathrm{N} 3-\mathrm{O} 9-\mathrm{O}^{\mathrm{vi}}$ & $155.54(18)$ \\
\hline $\mathrm{O} 4-\mathrm{Tm}-\mathrm{O} 5$ & $51.46(6)$ & $\mathrm{O} 8-\mathrm{O} 9-\mathrm{O}^{\mathrm{vi}}$ & $132.39(12)$ \\
\hline $\mathrm{O} 7-\mathrm{Tm}-\mathrm{O} 5$ & $69.56(6)$ & $\mathrm{O} 7-\mathrm{O} 9-\mathrm{O}^{\mathrm{vi}}$ & $154.89(11)$ \\
\hline
\end{tabular}




\begin{tabular}{|c|c|c|c|}
\hline $\mathrm{O} 2-\mathrm{Tm}-\mathrm{O} 5$ & $67.92(6)$ & $\mathrm{O} 15^{\mathrm{i}}-\mathrm{O} 9-\mathrm{O}^{\mathrm{vi}}$ & $70.34(7)$ \\
\hline $\mathrm{O} 10-\mathrm{Tm}-\mathrm{N} 1$ & $79.34(6)$ & $\mathrm{O} 9^{\mathrm{vi}}-\mathrm{O} 9-\mathrm{O}^{\mathrm{vi}}$ & $117.98(10)$ \\
\hline $\mathrm{O} 11-\mathrm{Tm}-\mathrm{N} 1$ & $94.01(6)$ & $\mathrm{Tm}-\mathrm{O} 10-\mathrm{O} 11$ & $54.60(5)$ \\
\hline $\mathrm{O} 12-\mathrm{Tm}-\mathrm{N} 1$ & $155.94(6)$ & $\mathrm{Tm}-\mathrm{O} 10-\mathrm{O} 15$ & $125.53(8)$ \\
\hline $\mathrm{O} 13-\mathrm{Tm}-\mathrm{N} 1$ & $94.17(6)$ & $\mathrm{O} 11-\mathrm{O} 10-\mathrm{O} 15$ & $72.21(7)$ \\
\hline $\mathrm{O} 1-\mathrm{Tm}-\mathrm{N} 1$ & $25.80(6)$ & $\mathrm{Tm}-\mathrm{O} 10-\mathrm{O} 14$ & $123.63(9)$ \\
\hline $\mathrm{O} 4-\mathrm{Tm}-\mathrm{N} 1$ & $128.96(6)$ & $\mathrm{O} 11-\mathrm{O} 10-\mathrm{O} 14$ & $170.18(10)$ \\
\hline $\mathrm{O} 7-\mathrm{Tm}-\mathrm{N} 1$ & $86.74(5)$ & $\mathrm{O} 15-\mathrm{O} 10-\mathrm{O} 14$ & $106.61(8)$ \\
\hline $\mathrm{O} 2-\mathrm{Tm}-\mathrm{N} 1$ & $25.70(6)$ & $\mathrm{Tm}-\mathrm{O} 10-\mathrm{O} 13$ & $54.55(5)$ \\
\hline $\mathrm{O} 5-\mathrm{Tm}-\mathrm{N} 1$ & $77.55(6)$ & $\mathrm{O} 11-\mathrm{O} 10-\mathrm{O} 13$ & $107.65(8)$ \\
\hline $\mathrm{O} 10-\mathrm{Tm}-\mathrm{N} 2$ & $143.67(6)$ & $\mathrm{O} 15-\mathrm{O} 10-\mathrm{O} 13$ & $179.02(11)$ \\
\hline $\mathrm{O} 11-\mathrm{Tm}-\mathrm{N} 2$ & $142.17(6)$ & $\mathrm{O} 14-\mathrm{O} 10-\mathrm{O} 13$ & $73.69(7)$ \\
\hline $\mathrm{O} 12-\mathrm{Tm}-\mathrm{N} 2$ & $96.51(6)$ & $\mathrm{Tm}-\mathrm{O} 10-\mathrm{O} 12$ & $52.28(5)$ \\
\hline $\mathrm{O} 13-\mathrm{Tm}-\mathrm{N} 2$ & $71.21(6)$ & $\mathrm{O} 11-\mathrm{O} 10-\mathrm{O} 12$ & $63.00(7)$ \\
\hline $\mathrm{O} 1-\mathrm{Tm}-\mathrm{N} 2$ & $113.66(7)$ & $\mathrm{O} 15-\mathrm{O} 10-\mathrm{O} 12$ & $114.74(9)$ \\
\hline $\mathrm{O} 4-\mathrm{Tm}-\mathrm{N} 2$ & $25.46(6)$ & $\mathrm{O} 14-\mathrm{O} 10-\mathrm{O} 12$ & $124.80(9)$ \\
\hline $\mathrm{O} 7-\mathrm{Tm}-\mathrm{N} 2$ & $73.26(6)$ & $\mathrm{O} 13-\mathrm{O} 10-\mathrm{O} 12$ & $64.45(7)$ \\
\hline $\mathrm{O} 2-\mathrm{Tm}-\mathrm{N} 2$ & $90.88(6)$ & $\mathrm{Tm}-\mathrm{O} 10-\mathrm{O} 2$ & $55.11(6)$ \\
\hline $\mathrm{O} 5-\mathrm{Tm}-\mathrm{N} 2$ & $26.04(6)$ & $\mathrm{O} 11-\mathrm{O} 10-\mathrm{O} 2$ & $92.39(8)$ \\
\hline $\mathrm{N} 1-\mathrm{Tm}-\mathrm{N} 2$ & $103.50(6)$ & $\mathrm{O} 15-\mathrm{O} 10-\mathrm{O} 2$ & $123.68(9)$ \\
\hline $\mathrm{O} 10-\mathrm{Tm}-\mathrm{O} 8$ & $128.95(6)$ & $\mathrm{O} 14-\mathrm{O} 10-\mathrm{O} 2$ & $80.12(8)$ \\
\hline $\mathrm{O} 11-\mathrm{Tm}-\mathrm{O} 8$ & $66.54(6)$ & $\mathrm{O} 13-\mathrm{O} 10-\mathrm{O} 2$ & $57.26(6)$ \\
\hline $\mathrm{O} 12-\mathrm{Tm}-\mathrm{O} 8$ & $66.66(6)$ & $\mathrm{O} 12-\mathrm{O} 10-\mathrm{O} 2$ & $103.77(8)$ \\
\hline $\mathrm{O} 13-\mathrm{Tm}-\mathrm{O} 8$ & $129.82(6)$ & $\mathrm{Tm}-\mathrm{O} 10-\mathrm{O} 1$ & $49.12(5)$ \\
\hline $\mathrm{O} 1-\mathrm{Tm}-\mathrm{O} 8$ & $108.14(5)$ & $\mathrm{O} 11-\mathrm{O} 10-\mathrm{O} 1$ & $55.52(6)$ \\
\hline $\mathrm{O} 4-\mathrm{Tm}-\mathrm{O} 8$ & $62.01(6)$ & $\mathrm{O} 15-\mathrm{O} 10-\mathrm{O} 1$ & $93.03(8)$ \\
\hline $\mathrm{O} 7-\mathrm{Tm}-\mathrm{O} 8$ & $45.43(5)$ & $\mathrm{O} 14-\mathrm{O} 10-\mathrm{O} 1$ & $115.31(9)$ \\
\hline $\mathrm{O} 2-\mathrm{Tm}-\mathrm{O} 8$ & $149.59(6)$ & $\mathrm{O} 13-\mathrm{O} 10-\mathrm{O} 1$ & $87.66(7)$ \\
\hline $\mathrm{O} 5-\mathrm{Tm}-\mathrm{O} 8$ & $92.10(6)$ & $\mathrm{O} 12-\mathrm{O} 10-\mathrm{O} 1$ & $97.74(7)$ \\
\hline $\mathrm{N} 1-\mathrm{Tm}-\mathrm{O} 8$ & $130.80(5)$ & $\mathrm{O} 2-\mathrm{O} 10-\mathrm{O} 1$ & $40.57(5)$ \\
\hline $\mathrm{N} 2-\mathrm{Tm}-\mathrm{O} 8$ & $76.75(5)$ & $\mathrm{Tm}-\mathrm{O} 10-\mathrm{H} 1$ & $130(3)$ \\
\hline $\mathrm{O} 10-\mathrm{Tm}-\mathrm{N} 3$ & $140.57(6)$ & $\mathrm{O} 11-\mathrm{O} 10-\mathrm{H} 1$ & $167(3)$ \\
\hline $\mathrm{O} 11-\mathrm{Tm}-\mathrm{N} 3$ & $68.94(6)$ & $\mathrm{O} 15-\mathrm{O} 10-\mathrm{H} 1$ & $100(3)$ \\
\hline $\mathrm{O} 12-\mathrm{Tm}-\mathrm{N} 3$ & $89.70(6)$ & $\mathrm{O} 14-\mathrm{O} 10-\mathrm{H} 1$ & $7(3)$ \\
\hline $\mathrm{O} 13-\mathrm{Tm}-\mathrm{N} 3$ & $141.77(6)$ & $\mathrm{O} 13-\mathrm{O} 10-\mathrm{H} 1$ & $81(3)$ \\
\hline $\mathrm{O} 1-\mathrm{Tm}-\mathrm{N} 3$ & $88.19(5)$ & $\mathrm{O} 12-\mathrm{O} 10-\mathrm{H} 1$ & $130(3)$ \\
\hline $\mathrm{O} 4-\mathrm{Tm}-\mathrm{N} 3$ & $68.85(6)$ & $\mathrm{O} 2-\mathrm{O} 10-\mathrm{H} 1$ & $84(3)$ \\
\hline $\mathrm{O} 7-\mathrm{Tm}-\mathrm{N} 3$ & $22.36(5)$ & $\mathrm{O} 1-\mathrm{O} 10-\mathrm{H} 1$ & $116(3)$ \\
\hline $\mathrm{O} 2-\mathrm{Tm}-\mathrm{N} 3$ & $126.79(6)$ & $\mathrm{Tm}-\mathrm{O} 10-\mathrm{H} 2$ & $120(3)$ \\
\hline $\mathrm{O} 5-\mathrm{Tm}-\mathrm{N} 3$ & $80.10(6)$ & $\mathrm{O} 11-\mathrm{O} 10-\mathrm{H} 2$ & $65(3)$ \\
\hline $\mathrm{N} 1-\mathrm{Tm}-\mathrm{N} 3$ & $108.51(5)$ & $\mathrm{O} 15-\mathrm{O} 10-\mathrm{H} 2$ & $11(3)$ \\
\hline $\mathrm{N} 2-\mathrm{Tm}-\mathrm{N} 3$ & $73.74(5)$ & $\mathrm{O} 14-\mathrm{O} 10-\mathrm{H} 2$ & $115(3)$ \\
\hline $\mathrm{O} 8-\mathrm{Tm}-\mathrm{N} 3$ & $23.07(5)$ & $\mathrm{O} 13-\mathrm{O} 10-\mathrm{H} 2$ & $168(3)$ \\
\hline $\mathrm{O} 3-\mathrm{N} 1-\mathrm{O} 2$ & $122.6(2)$ & $\mathrm{O} 12-\mathrm{O} 10-\mathrm{H} 2$ & $103(3)$ \\
\hline $\mathrm{O} 3-\mathrm{N} 1-\mathrm{O} 1$ & $121.5(2)$ & $\mathrm{O} 2-\mathrm{O} 10-\mathrm{H} 2$ & $131(3)$ \\
\hline $\mathrm{O} 2-\mathrm{N} 1-\mathrm{O} 1$ & $115.82(18)$ & $\mathrm{O} 1-\mathrm{O} 10-\mathrm{H} 2$ & $96(3)$ \\
\hline $\mathrm{O} 3-\mathrm{N} 1-\mathrm{Tm}$ & $177.14(17)$ & $\mathrm{H} 1-\mathrm{O} 10-\mathrm{H} 2$ & $108(4)$ \\
\hline $\mathrm{O} 2-\mathrm{N} 1-\mathrm{Tm}$ & $60.15(11)$ & $\mathrm{Tm}-\mathrm{O} 11-\mathrm{O} 15^{\mathrm{viii}}$ & $123.70(8)$ \\
\hline
\end{tabular}




\begin{tabular}{|c|c|}
\hline $\mathrm{O} 1-\mathrm{N} 1-\mathrm{Tm}$ & $55.68(10)$ \\
\hline $\mathrm{N} 1-\mathrm{O} 1-\mathrm{O} 2$ & 31.87 (11) \\
\hline $\mathrm{N} 1-\mathrm{O} 1-\mathrm{O} 3$ & $28.72(11)$ \\
\hline $\mathrm{O} 2-\mathrm{O} 1-\mathrm{O} 3$ & $60.58(9)$ \\
\hline $\mathrm{N} 1-\mathrm{O} 1-\mathrm{Tm}$ & $98.52(12)$ \\
\hline $\mathrm{O} 2-\mathrm{O} 1-\mathrm{Tm}$ & $66.66(7)$ \\
\hline $\mathrm{O} 3-\mathrm{O} 1-\mathrm{Tm}$ & $127.24(9)$ \\
\hline $\mathrm{N} 1-\mathrm{O} 1-\mathrm{O} 7$ & $128.53(15)$ \\
\hline $\mathrm{O} 2-\mathrm{O} 1-\mathrm{O} 7$ & $106.29(10)$ \\
\hline $\mathrm{O} 3-\mathrm{O} 1-\mathrm{O} 7$ & $140.60(12)$ \\
\hline $\mathrm{Tm}-\mathrm{O} 1-\mathrm{O} 7$ & $56.61(5)$ \\
\hline $\mathrm{N} 1-\mathrm{O} 1-\mathrm{O} 11$ & $139.90(14)$ \\
\hline $\mathrm{O} 2-\mathrm{O} 1-\mathrm{O} 11$ & $112.99(9)$ \\
\hline $\mathrm{O} 3-\mathrm{O} 1-\mathrm{O} 11$ & $155.01(11)$ \\
\hline $\mathrm{Tm}-\mathrm{O} 1-\mathrm{O} 11$ & $52.87(5)$ \\
\hline $\mathrm{O} 7-\mathrm{O} 1-\mathrm{O} 11$ & $63.47(6)$ \\
\hline $\mathrm{N} 1-\mathrm{O} 1-\mathrm{O} 11^{\mathrm{i}}$ & $140.49(14)$ \\
\hline $\mathrm{O} 2-\mathrm{O} 1-\mathrm{O} 11^{\mathrm{i}}$ & $158.78(12)$ \\
\hline $\mathrm{O} 3-\mathrm{O} 1-\mathrm{O} 11^{\mathrm{i}}$ & $115.14(9)$ \\
\hline $\mathrm{Tm}-\mathrm{O} 1-\mathrm{O} 11^{\mathrm{i}}$ & $114.13(7)$ \\
\hline $\mathrm{O} 7-\mathrm{O} 1-\mathrm{O} 11^{\mathrm{i}}$ & $62.71(6)$ \\
\hline $\mathrm{O} 11-\mathrm{O} 1-\mathrm{O} 11^{\mathrm{i}}$ & $79.48(7)$ \\
\hline $\mathrm{N} 1-\mathrm{O} 1-\mathrm{O} 10$ & 86.14 (13) \\
\hline $\mathrm{O} 2-\mathrm{O} 1-\mathrm{O} 10$ & $64.64(8)$ \\
\hline $\mathrm{O} 3-\mathrm{O} 1-\mathrm{O} 10$ & $106.16(9)$ \\
\hline $\mathrm{Tm}-\mathrm{O} 1-\mathrm{O} 10$ & $46.07(5)$ \\
\hline $\mathrm{O} 7-\mathrm{O} 1-\mathrm{O} 10$ & $98.82(7)$ \\
\hline $\mathrm{O} 11-\mathrm{O} 1-\mathrm{O} 10$ & $53.92(6)$ \\
\hline $\mathrm{O} 11^{\mathrm{i}}-\mathrm{O} 1-\mathrm{O} 10$ & $132.50(8)$ \\
\hline $\mathrm{N} 1-\mathrm{O} 1-\mathrm{O} 15^{\mathrm{ii}}$ & 89.65 (13) \\
\hline $\mathrm{O} 2-\mathrm{O} 1-\mathrm{O} 15^{\mathrm{ii}}$ & $117.05(9)$ \\
\hline $\mathrm{O} 3-\mathrm{O} 1-\mathrm{O} 15^{\mathrm{ii}}$ & $64.92(7)$ \\
\hline $\mathrm{Tm}-\mathrm{O} 1-\mathrm{O} 15^{\mathrm{ii}}$ & $150.29(9)$ \\
\hline $\mathrm{O} 7-\mathrm{O} 1-\mathrm{O} 15^{\mathrm{ii}}$ & $96.18(7)$ \\
\hline $\mathrm{O} 11-\mathrm{O} 1-\mathrm{O} 15^{\mathrm{ii}}$ & $129.57(8)$ \\
\hline $\mathrm{O} 11^{\mathrm{i}}-\mathrm{O} 1-\mathrm{O} 15^{\mathrm{ii}}$ & $51.03(5)$ \\
\hline $\mathrm{O} 10-\mathrm{O} 1-\mathrm{O} 15^{\mathrm{ii}}$ & $163.64(8)$ \\
\hline $\mathrm{N} 1-\mathrm{O} 2-\mathrm{O} 1$ & $32.31(11)$ \\
\hline $\mathrm{N} 1-\mathrm{O} 2-\mathrm{O} 3$ & $28.38(11)$ \\
\hline $\mathrm{O} 1-\mathrm{O} 2-\mathrm{O} 3$ & $60.69(8)$ \\
\hline $\mathrm{N} 1-\mathrm{O} 2-\mathrm{Tm}$ & 94.15 (13) \\
\hline $\mathrm{O} 1-\mathrm{O} 2-\mathrm{Tm}$ & $61.84(6)$ \\
\hline $\mathrm{O} 3-\mathrm{O} 2-\mathrm{Tm}$ & $122.53(9)$ \\
\hline $\mathrm{N} 1-\mathrm{O} 2-\mathrm{O} 13$ & $145.64(15)$ \\
\hline $\mathrm{O} 1-\mathrm{O} 2-\mathrm{O} 13$ & $113.94(9)$ \\
\hline $\mathrm{O} 3-\mathrm{O} 2-\mathrm{O} 13$ & $170.33(12)$ \\
\hline $\mathrm{Tm}-\mathrm{O} 2-\mathrm{O} 13$ & $52.70(5)$ \\
\hline $\mathrm{N} 1-\mathrm{O} 2-\mathrm{O} 5$ & $107.14(15)$ \\
\hline
\end{tabular}

\begin{tabular}{|c|c|}
\hline $\mathrm{Tm}-\mathrm{O} 11-\mathrm{O} 10$ & $53.37(5)$ \\
\hline $\mathrm{O} 15^{\mathrm{viii}}-\mathrm{O} 11-\mathrm{O} 10$ & $122.60(9)$ \\
\hline $\mathrm{Tm}-\mathrm{O} 11-\mathrm{O} 1$ & $55.51(5)$ \\
\hline $\mathrm{O} 15^{\mathrm{viii}-\mathrm{O} 11-\mathrm{O} 1}$ & $164.63(9)$ \\
\hline $\mathrm{O} 10-\mathrm{O} 11-\mathrm{O} 1$ & $70.56(7)$ \\
\hline $\mathrm{Tm}-\mathrm{O} 11-\mathrm{O} 7$ & $54.93(5)$ \\
\hline 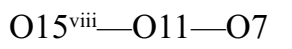 & $107.83(8)$ \\
\hline $\mathrm{O} 10-\mathrm{O} 11-\mathrm{O} 7$ & $106.57(8)$ \\
\hline $\mathrm{O} 1-\mathrm{O} 11-\mathrm{O} 7$ & $58.11(6)$ \\
\hline $\mathrm{Tm}-\mathrm{O} 11-\mathrm{O} 12$ & $51.22(5)$ \\
\hline $\mathrm{O} 15^{\mathrm{viii}}-\mathrm{O} 11-\mathrm{O} 12$ & $77.11(7)$ \\
\hline $\mathrm{O} 10-\mathrm{O} 11-\mathrm{O} 12$ & $60.99(6)$ \\
\hline $\mathrm{O} 1-\mathrm{O} 11-\mathrm{O} 12$ & $106.16(7)$ \\
\hline $\mathrm{O} 7-\mathrm{O} 11-\mathrm{O} 12$ & $86.16(7)$ \\
\hline $\mathrm{Tm}-\mathrm{O} 11-\mathrm{O} 8$ & $67.52(6)$ \\
\hline $\mathrm{O} 15^{\text {viii-O} 11-O 8}$ & $68.58(7)$ \\
\hline $\mathrm{O} 10-\mathrm{O} 11-\mathrm{O} 8$ & $114.24(8)$ \\
\hline $\mathrm{O} 1-\mathrm{O} 11-\mathrm{O} 8$ & $99.54(8)$ \\
\hline $\mathrm{O} 7-\mathrm{O} 11-\mathrm{O} 8$ & $43.17(6)$ \\
\hline $\mathrm{O} 12-\mathrm{O} 11-\mathrm{O} 8$ & $60.70(6)$ \\
\hline $\mathrm{Tm}-\mathrm{O} 11-\mathrm{O} 1^{\mathrm{i}}$ & $129.79(8)$ \\
\hline $\mathrm{O} 15^{\mathrm{vii}}-\mathrm{O} 11-\mathrm{O} 1^{\mathrm{i}}$ & $67.77(7)$ \\
\hline $\mathrm{O} 10-\mathrm{O} 11-\mathrm{O} 1^{\mathrm{i}}$ & $167.02(9)$ \\
\hline $\mathrm{O} 1-\mathrm{O} 11-\mathrm{O} 1^{\mathrm{i}}$ & $100.52(7)$ \\
\hline $\mathrm{O} 7-\mathrm{O} 11-\mathrm{O} 1^{\mathrm{i}}$ & $74.86(7)$ \\
\hline $\mathrm{O} 12-\mathrm{O} 11-\mathrm{O} 1^{\mathrm{i}}$ & $131.78(8)$ \\
\hline $\mathrm{O} 8-\mathrm{O} 11-\mathrm{O} 1^{\mathrm{i}}$ & $75.93(7)$ \\
\hline $\mathrm{Tm}-\mathrm{O} 11-\mathrm{O}^{\mathrm{i}}$ & $132.35(8)$ \\
\hline $\mathrm{O} 15^{\mathrm{viii}}-\mathrm{O} 11-\mathrm{O} 7^{\mathrm{i}}$ & $102.34(7)$ \\
\hline $\mathrm{O} 10-\mathrm{O} 11-\mathrm{O}^{\mathrm{i}}$ & $113.06(8)$ \\
\hline $\mathrm{O} 1-\mathrm{O} 11-\mathrm{O} 7^{\mathrm{i}}$ & $76.86(7)$ \\
\hline $\mathrm{O} 7-\mathrm{O} 11-\mathrm{O}^{\mathrm{i}}$ & $102.68(7)$ \\
\hline $\mathrm{O} 12-\mathrm{O} 11-\mathrm{O} 7^{\mathrm{i}}$ & $170.75(9)$ \\
\hline $\mathrm{O} 8-\mathrm{O} 11-\mathrm{O}^{\mathrm{i}}$ & $127.94(8)$ \\
\hline $\mathrm{O} 1^{\mathrm{i}-} \mathrm{O} 11-\mathrm{O}^{\mathrm{i}}$ & $54.64(5)$ \\
\hline $\mathrm{Tm}-\mathrm{O} 11-\mathrm{O} 15$ & $106.37(7)$ \\
\hline 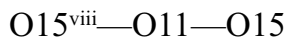 & $102.98(7)$ \\
\hline $\mathrm{O} 10-\mathrm{O} 11-\mathrm{O} 15$ & $53.90(6)$ \\
\hline $\mathrm{O} 1-\mathrm{O} 11-\mathrm{O} 15$ & $91.32(7)$ \\
\hline $\mathrm{O} 7-\mathrm{O} 11-\mathrm{O} 15$ & $149.18(8)$ \\
\hline $\mathrm{O} 12-\mathrm{O} 11-\mathrm{O} 15$ & $100.27(7)$ \\
\hline $\mathrm{O} 8-\mathrm{O} 11-\mathrm{O} 15$ & $160.04(8)$ \\
\hline $\mathrm{O} 1{ }^{\mathrm{i}}-\mathrm{O} 11-\mathrm{O} 15$ & $118.68(7)$ \\
\hline $\mathrm{O}^{\mathrm{i}}-\mathrm{O} 11-\mathrm{O} 15$ & $70.75(6)$ \\
\hline $\mathrm{Tm}-\mathrm{O} 11-\mathrm{H} 3$ & $129(3)$ \\
\hline $\mathrm{O} 15^{\mathrm{viii}-\mathrm{O} 11-\mathrm{H} 3}$ & $105(3)$ \\
\hline $\mathrm{O} 10-\mathrm{O} 11-\mathrm{H} 3$ & $113(3)$ \\
\hline $\mathrm{O} 1-\mathrm{O} 11-\mathrm{H} 3$ & $74(3)$ \\
\hline
\end{tabular}




\begin{tabular}{|c|c|c|c|}
\hline $\mathrm{O} 1-\mathrm{O} 2-\mathrm{O} 5$ & $87.66(9)$ & $\mathrm{O} 7-\mathrm{O} 11-\mathrm{H} 3$ & $99(3)$ \\
\hline $\mathrm{O} 3-\mathrm{O} 2-\mathrm{O} 5$ & $121.17(11)$ & $\mathrm{O} 12-\mathrm{O} 11-\mathrm{H} 3$ & $173(3)$ \\
\hline $\mathrm{Tm}-\mathrm{O} 2-\mathrm{O} 5$ & $56.41(5)$ & $\mathrm{O} 8-\mathrm{O} 11-\mathrm{H} 3$ & $126(3)$ \\
\hline $\mathrm{O} 13-\mathrm{O} 2-\mathrm{O} 5$ & $64.70(7)$ & $\mathrm{O} 11^{\mathrm{i}}-\mathrm{O} 11-\mathrm{H} 3$ & $54(3)$ \\
\hline $\mathrm{N} 1-\mathrm{O} 2-\mathrm{O} 10$ & $95.68(14)$ & $\mathrm{O} 7^{\mathrm{i}}-\mathrm{O} 11-\mathrm{H} 3$ & $4(3)$ \\
\hline $\mathrm{O} 1-\mathrm{O} 2-\mathrm{O} 10$ & $74.80(8)$ & $\mathrm{O} 15-\mathrm{O} 11-\mathrm{H} 3$ & $73(3)$ \\
\hline $\mathrm{O} 3-\mathrm{O} 2-\mathrm{O} 10$ & $113.21(10)$ & $\mathrm{Tm}-\mathrm{O} 11-\mathrm{H} 4$ & $121(3)$ \\
\hline $\mathrm{Tm}-\mathrm{O} 2-\mathrm{O} 10$ & $48.61(5)$ & 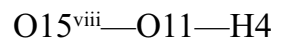 & $4(3)$ \\
\hline $\mathrm{O} 13-\mathrm{O} 2-\mathrm{O} 10$ & $57.13(6)$ & $\mathrm{O} 10-\mathrm{O} 11-\mathrm{H} 4$ & $118(3)$ \\
\hline $\mathrm{O} 5-\mathrm{O} 2-\mathrm{O} 10$ & $102.39(8)$ & $\mathrm{O} 1-\mathrm{O} 11-\mathrm{H} 4$ & $168(3)$ \\
\hline $\mathrm{N} 1-\mathrm{O} 2-\mathrm{O} 6^{\mathrm{iii}}$ & $118.54(14)$ & $\mathrm{O} 7-\mathrm{O} 11-\mathrm{H} 4$ & $110(3)$ \\
\hline $\mathrm{O} 1-\mathrm{O} 2-\mathrm{O} 6^{\mathrm{iii}}$ & $143.35(11)$ & $\mathrm{O} 12-\mathrm{O} 11-\mathrm{H} 4$ & $74(3)$ \\
\hline $\mathrm{O} 3-\mathrm{O} 2-\mathrm{O}^{\mathrm{iii}}$ & $93.77(9)$ & $\mathrm{O} 8-\mathrm{O} 11-\mathrm{H} 4$ & $69(3)$ \\
\hline $\mathrm{Tm}-\mathrm{O} 2-\mathrm{O}^{\mathrm{iii}}$ & $134.73(9)$ & $\mathrm{O} 1-\mathrm{i}-\mathrm{O} 11-\mathrm{H} 4$ & $72(3)$ \\
\hline $\mathrm{O} 13-\mathrm{O} 2-\mathrm{O} 6^{\mathrm{iii}}$ & $86.94(7)$ & 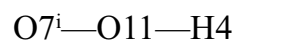 & $105(3)$ \\
\hline $\mathrm{O} 5-\mathrm{O} 2-\mathrm{O}^{\mathrm{iii}}$ & $128.98(8)$ & $\mathrm{O} 15-\mathrm{O} 11-\mathrm{H} 4$ & $101(3)$ \\
\hline $\mathrm{O} 10-\mathrm{O} 2-\mathrm{O}^{\mathrm{iii}}$ & $94.82(8)$ & $\mathrm{H} 3-\mathrm{O} 11-\mathrm{H} 4$ & $108(4)$ \\
\hline $\mathrm{N} 1-\mathrm{O} 2-\mathrm{O} 13^{\mathrm{iii}}$ & $119.36(15)$ & $\mathrm{Tm}-\mathrm{O} 12-\mathrm{O} 4$ & $55.65(5)$ \\
\hline $\mathrm{O} 1-\mathrm{O} 2-\mathrm{O} 13^{\mathrm{iii}}$ & $129.73(11)$ & $\mathrm{Tm}-\mathrm{O} 12-\mathrm{O} 10$ & $51.09(5)$ \\
\hline $\mathrm{O} 3-\mathrm{O} 2-\mathrm{O} 13^{\mathrm{iii}}$ & $103.41(9)$ & $\mathrm{O} 4-\mathrm{O} 12-\mathrm{O} 10$ & $101.51(7)$ \\
\hline $\mathrm{Tm}-\mathrm{O} 2-\mathrm{O} 13^{\mathrm{iii}}$ & $113.12(7)$ & $\mathrm{Tm}-\mathrm{O} 12-\mathrm{O} 11$ & $51.15(5)$ \\
\hline $\mathrm{O} 13-\mathrm{O} 2-\mathrm{O} 13^{\mathrm{iii}}$ & $86.20(7)$ & $\mathrm{O} 4-\mathrm{O} 12-\mathrm{O} 11$ & $96.35(7)$ \\
\hline $\mathrm{O} 5-\mathrm{O} 2-\mathrm{O} 13^{\mathrm{iii}}$ & $58.87(6)$ & $\mathrm{O} 10-\mathrm{O} 12-\mathrm{O} 11$ & $56.01(6)$ \\
\hline $\mathrm{O} 10-\mathrm{O} 2-\mathrm{O} 13^{\mathrm{iii}}$ & $143.20(8)$ & $\mathrm{Tm}-\mathrm{O} 12-\mathrm{O} 8^{\mathrm{vii}}$ & $121.45(8)$ \\
\hline $\mathrm{O} 6^{\mathrm{iii}}-\mathrm{O} 2-\mathrm{O} 13^{\mathrm{iii}}$ & $78.86(6)$ & $\mathrm{O} 4-\mathrm{O} 12-\mathrm{O}^{\mathrm{vii}}$ & $81.85(7)$ \\
\hline $\mathrm{N} 1-\mathrm{O} 3-\mathrm{O} 2$ & $28.99(12)$ & $\mathrm{O} 10-\mathrm{O} 12-\mathrm{O}^{\mathrm{vii}}$ & $161.11(9)$ \\
\hline $\mathrm{N} 1-\mathrm{O} 3-\mathrm{O} 1$ & $29.74(11)$ & $\mathrm{O} 11-\mathrm{O} 12-\mathrm{O}^{\mathrm{vii}}$ & $105.28(8)$ \\
\hline $\mathrm{O} 2-\mathrm{O} 3-\mathrm{O} 1$ & $58.73(8)$ & $\mathrm{Tm}-\mathrm{O} 12-\mathrm{O} 8$ & $67.41(6)$ \\
\hline $\mathrm{N} 1-\mathrm{O} 3-\mathrm{O} 14^{\mathrm{iv}}$ & $129.45(15)$ & $\mathrm{O} 4-\mathrm{O} 12-\mathrm{O} 8$ & $58.77(7)$ \\
\hline $\mathrm{O} 2-\mathrm{O} 3-\mathrm{O} 14^{\mathrm{iv}}$ & $112.88(10)$ & $\mathrm{O} 10-\mathrm{O} 12-\mathrm{O} 8$ & $109.76(8)$ \\
\hline $\mathrm{O} 1-\mathrm{O} 3-\mathrm{O} 14^{\mathrm{iv}}$ & $136.24(11)$ & $\mathrm{O} 11-\mathrm{O} 12-\mathrm{O} 8$ & $60.52(6)$ \\
\hline $\mathrm{N} 1-\mathrm{O} 3-\mathrm{O} 15^{\mathrm{ii}}$ & $99.15(14)$ & $\mathrm{O} 8^{\text {vii }-\mathrm{O} 12-\mathrm{O} 8}$ & $55.90(7)$ \\
\hline $\mathrm{O} 2-\mathrm{O} 3-\mathrm{O} 15^{\mathrm{ii}}$ & $123.07(9)$ & $\mathrm{Tm}-\mathrm{O} 12-\mathrm{O} 13$ & $50.30(5)$ \\
\hline $\mathrm{O} 1-\mathrm{O} 3-\mathrm{O} 15^{\mathrm{ii}}$ & $73.86(8)$ & $\mathrm{O} 4-\mathrm{O} 12-\mathrm{O} 13$ & $58.57(6)$ \\
\hline $\mathrm{O} 14^{\mathrm{iv}}-\mathrm{O} 3-\mathrm{O} 15^{\mathrm{ii}}$ & $123.21(8)$ & $\mathrm{O} 10-\mathrm{O} 12-\mathrm{O} 13$ & $55.69(6)$ \\
\hline $\mathrm{N} 1-\mathrm{O} 3-\mathrm{O} 14^{\mathrm{ii}}$ & $103.78(16)$ & $\mathrm{O} 11-\mathrm{O} 12-\mathrm{O} 13$ & $96.31(7)$ \\
\hline $\mathrm{O} 2-\mathrm{O} 3-\mathrm{O} 14^{\mathrm{ii}}$ & $86.31(9)$ & $\mathrm{O} 8{ }^{\mathrm{vii}}-\mathrm{O} 12-\mathrm{O} 13$ & $136.79(9)$ \\
\hline $\mathrm{O} 1-\mathrm{O} 3-\mathrm{O} 14^{\mathrm{ii}}$ & $119.12(10)$ & $\mathrm{O} 8-\mathrm{O} 12-\mathrm{O} 13$ & $108.33(8)$ \\
\hline $\mathrm{O} 14^{\mathrm{iv}}-\mathrm{O} 3-\mathrm{O} 14^{\mathrm{ii}}$ & $101.62(7)$ & $\mathrm{Tm}-\mathrm{O} 12-\mathrm{O} 9^{\mathrm{vii}}$ & $159.42(9)$ \\
\hline $\mathrm{O} 15^{\mathrm{ii}}-\mathrm{O} 3-\mathrm{O} 14^{\mathrm{ii}}$ & $91.31(7)$ & $\mathrm{O} 4-\mathrm{O} 12-\mathrm{O} 9^{\mathrm{vii}}$ & $105.31(8)$ \\
\hline $\mathrm{O} 6-\mathrm{N} 2-\mathrm{O} 4$ & $122.0(2)$ & $\mathrm{O} 10-\mathrm{O} 12-\mathrm{O} 9^{\mathrm{vii}}$ & $149.31(9)$ \\
\hline $\mathrm{O} 6-\mathrm{N} 2-\mathrm{O} 5$ & $122.5(2)$ & $\mathrm{O} 11-\mathrm{O} 12-\mathrm{O} 9^{\mathrm{vii}}$ & $133.55(8)$ \\
\hline $\mathrm{O} 4-\mathrm{N} 2-\mathrm{O} 5$ & $115.49(19)$ & $\mathrm{O} 8^{\mathrm{vii}}-\mathrm{O} 12-\mathrm{O} 9^{\mathrm{vii}}$ & $40.99(5)$ \\
\hline $\mathrm{O} 6-\mathrm{N} 2-\mathrm{Tm}$ & $175.95(18)$ & $\mathrm{O} 8-\mathrm{O} 12-\mathrm{O} 9^{\mathrm{vii}}$ & $96.86(7)$ \\
\hline $\mathrm{O} 4-\mathrm{N} 2-\mathrm{Tm}$ & $55.34(11)$ & $\mathrm{O} 13-\mathrm{O} 12-\mathrm{O} 9^{\mathrm{vii}}$ & $130.07(8)$ \\
\hline $\mathrm{O} 5-\mathrm{N} 2-\mathrm{Tm}$ & $60.28(11)$ & $\mathrm{Tm}-\mathrm{O} 12-\mathrm{H} 5$ & $124(3)$ \\
\hline $\mathrm{N} 2-\mathrm{O} 4-\mathrm{O} 5$ & $32.44(11)$ & $\mathrm{O} 4-\mathrm{O} 12-\mathrm{H} 5$ & $80(3)$ \\
\hline $\mathrm{N} 2-\mathrm{O} 4-\mathrm{O} 6$ & $28.47(12)$ & $\mathrm{O} 10-\mathrm{O} 12-\mathrm{H} 5$ & $167(3)$ \\
\hline
\end{tabular}




\begin{tabular}{|c|c|c|c|}
\hline $\mathrm{O} 5-\mathrm{O} 4-\mathrm{O} 6$ & $60.91(9)$ & $\mathrm{O} 11-\mathrm{O} 12-\mathrm{H} 5$ & $111(3)$ \\
\hline $\mathrm{N} 2-\mathrm{O} 4-\mathrm{Tm}$ & $99.20(13)$ & $\mathrm{O} 8^{\mathrm{vii}}-\mathrm{O} 12-\mathrm{H} 5$ & $6(3)$ \\
\hline $\mathrm{O} 5-\mathrm{O} 4-\mathrm{Tm}$ & $66.85(7)$ & $\mathrm{O} 8-\mathrm{O} 12-\mathrm{H} 5$ & $60(3)$ \\
\hline $\mathrm{O} 6-\mathrm{O} 4-\mathrm{Tm}$ & $127.60(9)$ & $\mathrm{O} 13-\mathrm{O} 12-\mathrm{H} 5$ & $133(3)$ \\
\hline $\mathrm{N} 2-\mathrm{O} 4-\mathrm{O} 12$ & $149.28(14)$ & $\mathrm{O} 9^{\mathrm{vii}}-\mathrm{O} 12-\mathrm{H} 5$ & $38(3)$ \\
\hline $\mathrm{O} 5-\mathrm{O} 4-\mathrm{O} 12$ & $118.57(9)$ & $\mathrm{Tm}-\mathrm{O} 12-\mathrm{H} 6$ & $124(3)$ \\
\hline $\mathrm{O} 6-\mathrm{O} 4-\mathrm{O} 12$ & $167.51(11)$ & $\mathrm{O} 4-\mathrm{O} 12-\mathrm{H} 6$ & $151(3)$ \\
\hline $\mathrm{Tm}-\mathrm{O} 4-\mathrm{O} 12$ & $52.63(5)$ & $\mathrm{O} 10-\mathrm{O} 12-\mathrm{H} 6$ & $73(3)$ \\
\hline $\mathrm{N} 2-\mathrm{O} 4-\mathrm{O} 13$ & $89.39(12)$ & $\mathrm{O} 11-\mathrm{O} 12-\mathrm{H} 6$ & $103(3)$ \\
\hline $\mathrm{O} 5-\mathrm{O} 4-\mathrm{O} 13$ & $71.81(8)$ & $\mathrm{O} 8^{\mathrm{vii}}-\mathrm{O} 12-\mathrm{H} 6$ & $113(3)$ \\
\hline $\mathrm{O} 6-\mathrm{O} 4-\mathrm{O} 13$ & $105.26(9)$ & $\mathrm{O} 8-\mathrm{O} 12-\mathrm{H} 6$ & $150(3)$ \\
\hline $\mathrm{Tm}-\mathrm{O} 4-\mathrm{O} 13$ & $52.19(5)$ & $\mathrm{O} 13-\mathrm{O} 12-\mathrm{H} 6$ & $98(3)$ \\
\hline $\mathrm{O} 12-\mathrm{O} 4-\mathrm{O} 13$ & $64.35(6)$ & $\mathrm{O} 9^{\mathrm{vii}}-\mathrm{O} 12-\mathrm{H} 6$ & $76(3)$ \\
\hline $\mathrm{N} 2-\mathrm{O} 4-\mathrm{O} 8$ & $121.99(15)$ & $\mathrm{H} 5-\mathrm{O} 12-\mathrm{H} 6$ & $112(4)$ \\
\hline $\mathrm{O} 5-\mathrm{O} 4-\mathrm{O} 8$ & $105.54(10)$ & $\mathrm{Tm}-\mathrm{O} 13-\mathrm{O} 14^{\mathrm{ix}}$ & $126.06(8)$ \\
\hline $\mathrm{O} 6-\mathrm{O} 4-\mathrm{O} 8$ & $128.45(10)$ & $\mathrm{Tm}-\mathrm{O} 13-\mathrm{O} 10$ & $52.99(5)$ \\
\hline $\mathrm{Tm}-\mathrm{O} 4-\mathrm{O} 8$ & $68.99(6)$ & $\mathrm{O} 14^{\mathrm{ix}}-\mathrm{O} 13-\mathrm{O} 10$ & $123.85(9)$ \\
\hline $\mathrm{O} 12-\mathrm{O} 4-\mathrm{O} 8$ & $64.03(7)$ & $\mathrm{Tm}-\mathrm{O} 13-\mathrm{O} 2$ & $58.48(5)$ \\
\hline $\mathrm{O} 13-\mathrm{O} 4-\mathrm{O} 8$ & $117.39(8)$ & $\mathrm{O} 14^{\mathrm{ix}}-\mathrm{O} 13-\mathrm{O} 2$ & $170.54(10)$ \\
\hline $\mathrm{N} 2-\mathrm{O} 4-\mathrm{O} 15^{\mathrm{v}}$ & $105.48(13)$ & $\mathrm{O} 10-\mathrm{O} 13-\mathrm{O} 2$ & $65.61(7)$ \\
\hline $\mathrm{O} 5-\mathrm{O} 4-\mathrm{O} 15^{\mathrm{v}}$ & $132.47(10)$ & $\mathrm{Tm}-\mathrm{O} 13-\mathrm{O} 4$ & $54.86(5)$ \\
\hline $\mathrm{O} 6-\mathrm{O} 4-\mathrm{O} 15^{\mathrm{v}}$ & $80.38(8)$ & $\mathrm{O} 14^{\mathrm{ix}}-\mathrm{O} 13-\mathrm{O} 4$ & $82.46(7)$ \\
\hline $\mathrm{Tm}-\mathrm{O} 4-\mathrm{O} 15^{\mathrm{v}}$ & $144.77(8)$ & $\mathrm{O} 10-\mathrm{O} 13-\mathrm{O} 4$ & $103.61(7)$ \\
\hline $\mathrm{O} 12-\mathrm{O} 4-\mathrm{O} 15^{\mathrm{v}}$ & $105.17(7)$ & $\mathrm{O} 2-\mathrm{O} 13-\mathrm{O} 4$ & $95.85(8)$ \\
\hline $\mathrm{O} 13-\mathrm{O} 4-\mathrm{O} 15^{\mathrm{v}}$ & $150.26(9)$ & $\mathrm{Tm}-\mathrm{O} 13-\mathrm{O} 5^{\mathrm{iii}}$ & $128.52(8)$ \\
\hline $\mathrm{O} 8-\mathrm{O} 4-\mathrm{O} 15^{\mathrm{v}}$ & $76.65(7)$ & $\mathrm{O} 14^{\mathrm{ix}}-\mathrm{O} 13-\mathrm{O} 5^{\mathrm{iii}}$ & $103.47(7)$ \\
\hline $\mathrm{N} 2-\mathrm{O} 4-\mathrm{O} 7$ & $84.55(14)$ & $\mathrm{O} 10-\mathrm{O} 13-\mathrm{O} 5^{\mathrm{iii}}$ & $112.21(8)$ \\
\hline $\mathrm{O} 5-\mathrm{O} 4-\mathrm{O} 7$ & $62.95(8)$ & $\mathrm{O} 2-\mathrm{O} 13-\mathrm{O} 5^{\mathrm{iii}}$ & $70.49(7)$ \\
\hline $\mathrm{O} 6-\mathrm{O} 4-\mathrm{O} 7$ & $104.27(9)$ & $\mathrm{O} 4-\mathrm{O} 13-\mathrm{O} 5^{\mathrm{iii}}$ & $130.25(8)$ \\
\hline $\mathrm{Tm}-\mathrm{O} 4-\mathrm{O} 7$ & $51.53(5)$ & $\mathrm{Tm}-\mathrm{O} 13-\mathrm{O} 5$ & $55.31(5)$ \\
\hline $\mathrm{O} 12-\mathrm{O} 4-\mathrm{O} 7$ & $85.18(7)$ & $\mathrm{O} 14^{\mathrm{ix}}-\mathrm{O} 13-\mathrm{O} 5$ & $115.55(8)$ \\
\hline $\mathrm{O} 13-\mathrm{O} 4-\mathrm{O} 7$ & $100.94(7)$ & $\mathrm{O} 10-\mathrm{O} 13-\mathrm{O} 5$ & $104.14(7)$ \\
\hline $\mathrm{O} 8-\mathrm{O} 4-\mathrm{O} 7$ & $42.60(6)$ & $\mathrm{O} 2-\mathrm{O} 13-\mathrm{O} 5$ & $58.80(7)$ \\
\hline $\mathrm{O} 15^{\mathrm{v}}-\mathrm{O} 4-\mathrm{O} 7$ & $105.93(7)$ & $\mathrm{O} 4-\mathrm{O} 13-\mathrm{O} 5$ & $43.43(6)$ \\
\hline $\mathrm{N} 2-\mathrm{O} 5-\mathrm{O} 4$ & $32.07(11)$ & $\mathrm{O} 5{ }^{\mathrm{iii}}-\mathrm{O} 13-\mathrm{O} 5$ & $93.63(7)$ \\
\hline $\mathrm{N} 2-\mathrm{O} 5-\mathrm{O} 6$ & $28.04(11)$ & $\mathrm{Tm}-\mathrm{O} 13-\mathrm{O} 12$ & $50.06(5)$ \\
\hline $\mathrm{O} 4-\mathrm{O} 5-\mathrm{O} 6$ & $60.10(8)$ & $\mathrm{O} 14^{\mathrm{ix}}-\mathrm{O} 13-\mathrm{O} 12$ & $80.61(7)$ \\
\hline $\mathrm{N} 2-\mathrm{O} 5-\mathrm{Tm}$ & $93.68(13)$ & $\mathrm{O} 10-\mathrm{O} 13-\mathrm{O} 12$ & $59.86(6)$ \\
\hline $\mathrm{O} 4-\mathrm{O} 5-\mathrm{Tm}$ & $61.69(7)$ & $\mathrm{O} 2-\mathrm{O} 13-\mathrm{O} 12$ & $106.36(7)$ \\
\hline $\mathrm{O} 6-\mathrm{O} 5-\mathrm{Tm}$ & $121.66(9)$ & $\mathrm{O} 4-\mathrm{O} 13-\mathrm{O} 12$ & $57.07(6)$ \\
\hline $\mathrm{N} 2-\mathrm{O} 5-\mathrm{O} 2$ & $138.74(15)$ & $\mathrm{O} 5{ }^{\mathrm{iii}}-\mathrm{O} 13-\mathrm{O} 12$ & $171.66(8)$ \\
\hline $\mathrm{O} 4-\mathrm{O} 5-\mathrm{O} 2$ & $112.22(9)$ & $\mathrm{O} 5-\mathrm{O} 13-\mathrm{O} 12$ & $91.06(7)$ \\
\hline $\mathrm{O} 6-\mathrm{O} 5-\mathrm{O} 2$ & $153.44(11)$ & $\mathrm{Tm}-\mathrm{O} 13-\mathrm{O} 2^{\mathrm{iii}}$ & $119.90(8)$ \\
\hline $\mathrm{Tm}-\mathrm{O} 5-\mathrm{O} 2$ & $55.67(5)$ & $\mathrm{O} 14^{\mathrm{ix}}-\mathrm{O} 13-\mathrm{O} 2^{\mathrm{iii}}$ & $76.74(7)$ \\
\hline $\mathrm{N} 2-\mathrm{O} 5-\mathrm{O} 7$ & $95.06(13)$ & $\mathrm{O} 10-\mathrm{O} 13-\mathrm{O} 2^{\mathrm{iii}}$ & $159.18(9)$ \\
\hline $\mathrm{O} 4-\mathrm{O} 5-\mathrm{O} 7$ & $74.85(8)$ & $\mathrm{O} 2-\mathrm{O} 13-\mathrm{O} 2^{\mathrm{iii}}$ & $93.80(7)$ \\
\hline $\mathrm{O} 6-\mathrm{O} 5-\mathrm{O} 7$ & $111.87(9)$ & $\mathrm{O} 4-\mathrm{O} 13-\mathrm{O} 2^{\mathrm{iii}}$ & $80.58(7)$ \\
\hline $\mathrm{Tm}-\mathrm{O} 5-\mathrm{O} 7$ & $54.27(5)$ & $\mathrm{O} 5^{\mathrm{iii}}-\mathrm{O} 13-\mathrm{O} 2^{\mathrm{iii}}$ & $54.24(6)$ \\
\hline
\end{tabular}




\begin{tabular}{|c|c|c|c|}
\hline $\mathrm{O} 2-\mathrm{O} 5-\mathrm{O} 7$ & $88.06(7)$ & $\mathrm{O} 5-\mathrm{O} 13-\mathrm{O} 2^{\mathrm{iii}}$ & $64.66(6)$ \\
\hline $\mathrm{N} 2-\mathrm{O} 5-\mathrm{O} 13^{\mathrm{iii}}$ & $122.46(14)$ & $\mathrm{O} 12-\mathrm{O} 13-\mathrm{O} 2^{\mathrm{iii}}$ & $134.09(8)$ \\
\hline $\mathrm{O} 4-\mathrm{O} 5-\mathrm{O} 13^{\mathrm{iii}}$ & $139.71(10)$ & $\mathrm{Tm}-\mathrm{O} 13-\mathrm{H} 7$ & $122(3)$ \\
\hline $\mathrm{O} 6-\mathrm{O} 5-\mathrm{O} 13^{\mathrm{iii}}$ & $101.74(9)$ & $\mathrm{O} 14^{\mathrm{ix}}-\mathrm{O} 13-\mathrm{H} 7$ & $5(3)$ \\
\hline $\mathrm{Tm}-\mathrm{O} 5-\mathrm{O} 13^{\mathrm{iii}}$ & $120.11(8)$ & $\mathrm{O} 10-\mathrm{O} 13-\mathrm{H} 7$ & $122(3)$ \\
\hline $\mathrm{O} 2-\mathrm{O} 5-\mathrm{O} 13^{\mathrm{iii}}$ & $66.89(6)$ & $\mathrm{O} 2-\mathrm{O} 13-\mathrm{H} 7$ & $171(3)$ \\
\hline $\mathrm{O} 7-\mathrm{O} 5-\mathrm{O} 13^{\mathrm{iii}}$ & $142.29(8)$ & $\mathrm{O} 4-\mathrm{O} 13-\mathrm{H} 7$ & $78(3)$ \\
\hline $\mathrm{N} 2-\mathrm{O} 5-\mathrm{O} 13$ & $82.93(13)$ & $\mathrm{O} 5{ }^{\mathrm{iii}-\mathrm{O} 13-\mathrm{H} 7}$ & $108(3)$ \\
\hline $\mathrm{O} 4-\mathrm{O} 5-\mathrm{O} 13$ & $64.76(7)$ & $\mathrm{O} 5-\mathrm{O} 13-\mathrm{H} 7$ & $113(3)$ \\
\hline $\mathrm{O} 6-\mathrm{O} 5-\mathrm{O} 13$ & $100.34(9)$ & $\mathrm{O} 12-\mathrm{O} 13-\mathrm{H} 7$ & $77(3)$ \\
\hline $\mathrm{Tm}-\mathrm{O} 5-\mathrm{O} 13$ & $49.52(5)$ & $\mathrm{O} 2{ }^{\mathrm{iii}}-\mathrm{O} 13-\mathrm{H} 7$ & $78(3)$ \\
\hline $\mathrm{O} 2-\mathrm{O} 5-\mathrm{O} 13$ & $56.50(6)$ & $\mathrm{Tm}-\mathrm{O} 13-\mathrm{H} 8$ & $129(3)$ \\
\hline $\mathrm{O} 7-\mathrm{O} 5-\mathrm{O} 13$ & $103.35(7)$ & $\mathrm{O} 14^{\mathrm{ix}}-\mathrm{O} 13-\mathrm{H} 8$ & $103(3)$ \\
\hline $\mathrm{O} 13^{\mathrm{iii}-\mathrm{O} 5-\mathrm{O} 13}$ & $86.37(7)$ & $\mathrm{O} 10-\mathrm{O} 13-\mathrm{H} 8$ & $113(3)$ \\
\hline $\mathrm{N} 2-\mathrm{O} 6-\mathrm{O} 4$ & $29.55(12)$ & $\mathrm{O} 2-\mathrm{O} 13-\mathrm{H} 8$ & $71(3)$ \\
\hline $\mathrm{N} 2-\mathrm{O} 6-\mathrm{O} 5$ & $29.44(12)$ & $\mathrm{O} 4-\mathrm{O} 13-\mathrm{H} 8$ & $130(3)$ \\
\hline $\mathrm{O} 4-\mathrm{O} 6-\mathrm{O} 5$ & $58.99(8)$ & $\mathrm{O}^{\mathrm{iii}-\mathrm{O} 13-\mathrm{H} 8}$ & $1(3)$ \\
\hline $\mathrm{N} 2-\mathrm{O} 6-\mathrm{O} 14^{\mathrm{iii}}$ & $116.45(16)$ & $\mathrm{O} 5-\mathrm{O} 13-\mathrm{H} 8$ & $94(3)$ \\
\hline $\mathrm{O} 4-\mathrm{O} 6-\mathrm{O} 14^{\mathrm{iii}}$ & $146.00(11)$ & $\mathrm{O} 12-\mathrm{O} 13-\mathrm{H} 8$ & $172(3)$ \\
\hline $\mathrm{O} 5-\mathrm{O} 6-\mathrm{O} 14^{\mathrm{iii}}$ & $87.01(9)$ & $\mathrm{O} 2{ }^{\mathrm{iii}}-\mathrm{O} 13-\mathrm{H} 8$ & $54(3)$ \\
\hline $\mathrm{N} 2-\mathrm{O} 6-09^{\mathrm{vi}}$ & $83.84(14)$ & $\mathrm{H} 7-\mathrm{O} 13-\mathrm{H} 8$ & $107(4)$ \\
\hline $\mathrm{O} 4-\mathrm{O} 6-\mathrm{O} 9^{\mathrm{vi}}$ & $77.76(9)$ & $\mathrm{O} 13^{\mathrm{ix}-\mathrm{O} 14-\mathrm{O} 10}$ & $98.12(8)$ \\
\hline $\mathrm{O} 5-\mathrm{O} 6-\mathrm{O} 9^{\mathrm{vi}}$ & $91.17(9)$ & $\mathrm{O} 13^{\mathrm{ix}}-\mathrm{O} 14-\mathrm{O}^{\mathrm{iii}}$ & $118.03(9)$ \\
\hline $\mathrm{O} 14^{\mathrm{iii}}-\mathrm{O} 6-\mathrm{O} 9^{\mathrm{vi}}$ & $104.35(9)$ & $\mathrm{O} 10-\mathrm{O} 14-\mathrm{O}^{\mathrm{iii}}$ & $107.66(9)$ \\
\hline $\mathrm{N} 2-\mathrm{O} 6-\mathrm{O} 2^{\mathrm{iii}}$ & $83.25(14)$ & $\mathrm{O} 13^{\mathrm{ix}}-\mathrm{O} 14-\mathrm{O}^{\mathrm{iv}}$ & $112.05(8)$ \\
\hline $\mathrm{O} 4-\mathrm{O} 6-\mathrm{O} 2^{\mathrm{iii}}$ & $93.56(9)$ & $\mathrm{O} 10-\mathrm{O} 14-\mathrm{O}^{\mathrm{iv}}$ & $139.09(9)$ \\
\hline $\mathrm{O} 5-\mathrm{O} 6-\mathrm{O} 2^{\mathrm{iii}}$ & $74.80(8)$ & $\mathrm{O}^{6 i i}-\mathrm{O} 14-\mathrm{O}^{\mathrm{iv}}$ & $82.46(7)$ \\
\hline $\mathrm{O} 14^{\mathrm{iii}}-\mathrm{O} 6-\mathrm{O} 2^{\mathrm{iii}}$ & $76.63(7)$ & $\mathrm{O} 13^{\mathrm{ix}}-\mathrm{O} 14-\mathrm{O} 3^{\mathrm{x}}$ & $93.53(8)$ \\
\hline $\mathrm{O} 9^{\mathrm{vi}}-\mathrm{O} 6-\mathrm{O} 2^{\mathrm{iii}}$ & $165.92(9)$ & $\mathrm{O} 10-\mathrm{O} 14-\mathrm{O}^{\mathrm{x}}$ & $72.61(7)$ \\
\hline $\mathrm{O} 9-\mathrm{N} 3-\mathrm{O} 8$ & $121.2(2)$ & 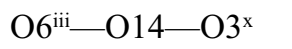 & $147.52(9)$ \\
\hline $\mathrm{O} 9-\mathrm{N} 3-\mathrm{O} 7$ & $119.94(19)$ & $\mathrm{O} 3^{\mathrm{iv}}-\mathrm{O} 14-\mathrm{O} 3^{\mathrm{x}}$ & $78.38(7)$ \\
\hline $\mathrm{O} 8-\mathrm{N} 3-\mathrm{O} 7$ & $118.84(19)$ & $\mathrm{O} 13^{\mathrm{ix}}-\mathrm{O} 14-\mathrm{O} 13$ & $69.68(7)$ \\
\hline $\mathrm{O} 9-\mathrm{N} 3-\mathrm{Tm}$ & $167.34(15)$ & $\mathrm{O} 10-\mathrm{O} 14-\mathrm{O} 13$ & $53.22(6)$ \\
\hline $\mathrm{O} 8-\mathrm{N} 3-\mathrm{Tm}$ & $71.43(13)$ & $\mathrm{O}^{\mathrm{iii}}-\mathrm{O} 14-\mathrm{O} 13$ & $82.55(7)$ \\
\hline $\mathrm{O} 7-\mathrm{N} 3-\mathrm{Tm}$ & $47.41(10)$ & $\mathrm{O}^{\mathrm{iv}}-\mathrm{O} 14-\mathrm{O} 13$ & $163.54(9)$ \\
\hline $\mathrm{N} 3-\mathrm{O} 7-\mathrm{O} 8$ & $30.06(11)$ & $\mathrm{O} 3^{\mathrm{x}}-\mathrm{O} 14-\mathrm{O} 13$ & $118.06(7)$ \\
\hline $\mathrm{N} 3-\mathrm{O} 7-\mathrm{O} 9$ & $29.46(11)$ & $\mathrm{O} 13^{\mathrm{ix}}-\mathrm{O} 14-\mathrm{H} 9$ & $116(3)$ \\
\hline $\mathrm{O} 8-\mathrm{O} 7-\mathrm{O} 9$ & $59.52(9)$ & $\mathrm{O} 10-\mathrm{O} 14-\mathrm{H} 9$ & $109(3)$ \\
\hline $\mathrm{N} 3-\mathrm{O} 7-\mathrm{Tm}$ & $110.23(13)$ & $\mathrm{O} 6^{\mathrm{iii}-\mathrm{O} 14-\mathrm{H} 9}$ & $3(3)$ \\
\hline $\mathrm{O} 8-\mathrm{O} 7-\mathrm{Tm}$ & $80.17(8)$ & $\mathrm{O}^{\mathrm{iv}}-\mathrm{O} 14-\mathrm{H} 9$ & $83(3)$ \\
\hline $\mathrm{O} 9-\mathrm{O} 7-\mathrm{Tm}$ & $139.69(10)$ & $\mathrm{O}^{\mathrm{x}}-\mathrm{O} 14-\mathrm{H} 9$ & $150(3)$ \\
\hline $\mathrm{N} 3-\mathrm{O} 7-\mathrm{O} 1$ & $147.87(15)$ & $\mathrm{O} 13-\mathrm{O} 14-\mathrm{H} 9$ & $82(3)$ \\
\hline $\mathrm{O} 8-\mathrm{O} 7-\mathrm{O} 1$ & $125.43(10)$ & $\mathrm{O} 13^{\mathrm{ix}-\mathrm{O} 14-\mathrm{H} 10}$ & $114(3)$ \\
\hline $\mathrm{O} 9-\mathrm{O} 7-\mathrm{O} 1$ & $152.51(11)$ & $\mathrm{O} 10-\mathrm{O} 14-\mathrm{H} 10$ & $111(3)$ \\
\hline $\mathrm{Tm}-\mathrm{O} 7-\mathrm{O} 1$ & $54.43(5)$ & $\mathrm{O}^{\mathrm{iii}}-\mathrm{O} 14-\mathrm{H} 10$ & $108(3)$ \\
\hline $\mathrm{N} 3-\mathrm{O} 7-\mathrm{O} 5$ & $121.52(14)$ & $\mathrm{O}^{\mathrm{iv}}-\mathrm{O} 14-\mathrm{H} 10$ & $31(3)$ \\
\hline $\mathrm{O} 8-\mathrm{O} 7-\mathrm{O} 5$ & $104.49(9)$ & $\mathrm{O} 3^{x}-\mathrm{O} 14-\mathrm{H} 10$ & $48(3)$ \\
\hline $\mathrm{O} 9-\mathrm{O} 7-\mathrm{O} 5$ & $130.94(10)$ & $\mathrm{O} 13-\mathrm{O} 14-\mathrm{H} 10$ & $164(3)$ \\
\hline
\end{tabular}




$$
\begin{aligned}
& \mathrm{Tm}-\mathrm{O} 7-\mathrm{O} 5 \\
& \mathrm{O} 1-\mathrm{O} 7-\mathrm{O} 5 \\
& \text { N3-O7-O11 } \\
& \mathrm{O} 8-\mathrm{O} 7-\mathrm{O} 11 \\
& \text { O9-O7-O11 } \\
& \mathrm{Tm}-\mathrm{O} 7-\mathrm{O} 11 \\
& \mathrm{O} 1-\mathrm{O} 7-\mathrm{O} 11 \\
& \text { O5-O7-O11 } \\
& \text { N3-O7-O11 } \\
& \text { O8-O7-O11 } \\
& \text { O9-07-O11 } \\
& \mathrm{Tm}-\mathrm{O} 7-\mathrm{O} 11^{\mathrm{i}} \\
& \mathrm{O} 1-\mathrm{O} 7-\mathrm{O} 11^{\mathrm{i}} \\
& \text { O5-O7-O11 } \\
& \mathrm{O} 11-\mathrm{O} 7-\mathrm{O} 11^{\mathrm{i}} \\
& \mathrm{N} 3-\mathrm{O} 7-\mathrm{O} 4 \\
& \mathrm{O} 8-\mathrm{O} 7-\mathrm{O} 4 \\
& \text { O9- } 07-\mathrm{O} 4 \\
& \mathrm{Tm}-\mathrm{O} 7-\mathrm{O} 4 \\
& \mathrm{O} 1-\mathrm{O} 7-\mathrm{O} 4 \\
& \text { O5-O7-O4 } \\
& \text { O11-O7-O4 } \\
& \text { O11-O } 7-\mathrm{O} 4 \\
& \text { N3-O8-O9 } \\
& \text { N3-O8-O7 } \\
& \text { O9-08-O7 } \\
& \mathrm{N} 3-\mathrm{O} 8-\mathrm{O}^{\mathrm{vii}} \\
& \mathrm{O} 9-\mathrm{O} 8-\mathrm{O}^{\mathrm{vii}} \\
& \mathrm{O} 7-\mathrm{O} 8-\mathrm{O} 8^{\mathrm{vii}} \\
& \text { N3-O8-O4 } \\
& \text { O9-O8-O4 } \\
& \text { O7-O8-O4 } \\
& \mathrm{O}^{\mathrm{vii}}-\mathrm{O} 8-\mathrm{O} 4 \\
& \mathrm{~N} 3-\mathrm{O} 8-\mathrm{O} 12^{\mathrm{vii}} \\
& \mathrm{O} 9-\mathrm{O} 8-\mathrm{O} 12^{\mathrm{vi}} \\
& \mathrm{O} 7-\mathrm{O} 8-\mathrm{O} 12^{\mathrm{vii}} \\
& \mathrm{O} 8^{\mathrm{vii}}-\mathrm{O} 8-\mathrm{O} 12^{\mathrm{vii}} \\
& \mathrm{O} 4-\mathrm{O} 8-\mathrm{O} 12^{\mathrm{vii}} \\
& \text { N3-O8-O11 } \\
& \mathrm{O} 9-\mathrm{O} 8-\mathrm{O} 11 \\
& \mathrm{O} 7-\mathrm{O} 8-\mathrm{O} 11 \\
& \text { O } 8 \text { vii-O8-O11 } \\
& \mathrm{O} 4-\mathrm{O} 8-\mathrm{O} 11 \\
& \mathrm{O} 12^{\mathrm{vii}}-\mathrm{O} 8-\mathrm{O} 11
\end{aligned}
$$

$56.17(5)$

\begin{tabular}{|c|c|}
\hline H9-O14-H10 & $109(4)$ \\
\hline $\mathrm{O} 11^{\text {viii }}-\mathrm{O} 15-\mathrm{O} 10$ & $105.91(8)$ \\
\hline $\mathrm{O} 11^{\mathrm{viii}}-\mathrm{O} 15-\mathrm{O} 9^{\mathrm{i}}$ & $123.52(9)$ \\
\hline $\mathrm{O} 10-\mathrm{O} 15-09^{\mathrm{i}}$ & $97.93(9)$ \\
\hline 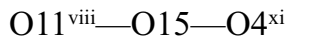 & $135.84(8)$ \\
\hline $\mathrm{O} 10-\mathrm{O} 15-\mathrm{O} 4^{\mathrm{xi}}$ & $113.20(8)$ \\
\hline $\mathrm{O} 9-\mathrm{O} 15-\mathrm{O} 4^{\mathrm{xi}}$ & $71.01(7)$ \\
\hline $\mathrm{O} 11^{\text {viii }}-\mathrm{O} 15-\mathrm{O} 3^{\mathrm{x}}$ & $101.79(8)$ \\
\hline $\mathrm{O} 10-\mathrm{O} 15-\mathrm{O}^{\mathrm{x}}$ & $74.76(7)$ \\
\hline $\mathrm{O} 9^{\mathrm{i}}-\mathrm{O} 15-\mathrm{O} 3^{\mathrm{x}}$ & $133.98(8)$ \\
\hline $\mathrm{O} 4^{\mathrm{xi}}-\mathrm{O} 15-\mathrm{O} 3^{\mathrm{x}}$ & $70.82(7)$ \\
\hline $\mathrm{O} 11^{\text {viii }}-\mathrm{O} 15-\mathrm{O} 1^{\mathrm{x}}$ & $61.19(6)$ \\
\hline $\mathrm{O} 10-\mathrm{O} 15-\mathrm{O} 1^{\mathrm{x}}$ & $80.98(7)$ \\
\hline 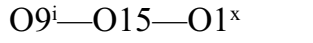 & $175.19(8)$ \\
\hline $\mathrm{O} 4^{\mathrm{xi}}-\mathrm{O} 15-\mathrm{O} 1^{\mathrm{x}}$ & $105.04(8)$ \\
\hline $\mathrm{O} 3^{\mathrm{x}}-\mathrm{O} 15-\mathrm{O} 1^{\mathrm{x}}$ & $41.22(5)$ \\
\hline 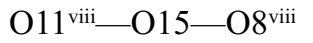 & $60.23(6)$ \\
\hline $\mathrm{O} 10-\mathrm{O} 15-\mathrm{O} 8^{\mathrm{viii}}$ & $151.59(9)$ \\
\hline $\mathrm{O} 9 \mathrm{i}-\mathrm{O} 15-\mathrm{O} 8^{\mathrm{viii}}$ & $110.39(8)$ \\
\hline $\mathrm{O} 4^{\mathrm{xi}}-\mathrm{O} 15-\mathrm{O}^{\mathrm{viii}}$ & $75.62(6)$ \\
\hline $\mathrm{O} 3^{\mathrm{x}}-\mathrm{O} 15-\mathrm{O} 8^{\mathrm{viii}}$ & $83.73(7)$ \\
\hline $\mathrm{O} 1^{x}-\mathrm{O} 15-\mathrm{O} 8^{\text {viii }}$ & $70.61(6)$ \\
\hline 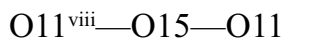 & $77.02(7)$ \\
\hline $\mathrm{O} 10-\mathrm{O} 15-\mathrm{O} 11$ & $53.89(6)$ \\
\hline O9'-O15-O11 & $77.44(7)$ \\
\hline $\mathrm{O} 4^{\mathrm{xi}}-\mathrm{O} 15-\mathrm{O} 11$ & $143.83(8)$ \\
\hline $\mathrm{O} 3^{\mathrm{x}}-\mathrm{O} 15-\mathrm{O} 11$ & $124.74(8)$ \\
\hline $\mathrm{O} 1{ }^{\mathrm{x}}-\mathrm{O} 15-\mathrm{O} 11$ & $105.40(7)$ \\
\hline $\mathrm{O} 88^{\mathrm{viii}}-\mathrm{O} 15-\mathrm{O} 11$ & $133.51(8)$ \\
\hline 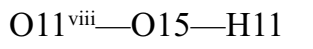 & $116(3)$ \\
\hline $\mathrm{O} 10-\mathrm{O} 15-\mathrm{H} 11$ & $105(3)$ \\
\hline $\mathrm{O} 9 \mathrm{i}-\mathrm{O} 15-\mathrm{H} 11$ & $8(3)$ \\
\hline $\mathrm{O} 4^{\mathrm{xi}}-\mathrm{O} 15-\mathrm{H} 11$ & $73(3)$ \\
\hline $\mathrm{O} 3^{\mathrm{x}}-\mathrm{O} 15-\mathrm{H} 11$ & $140(3)$ \\
\hline $\mathrm{O} 1^{\mathrm{x}}-\mathrm{O} 15-\mathrm{H} 11$ & $174(3)$ \\
\hline $\mathrm{O} 8^{\text {viii-OO15-H11 }}$ & $104(3)$ \\
\hline O11—O15-H11 & $78(3)$ \\
\hline 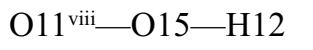 & $116(3)$ \\
\hline $\mathrm{O} 10-\mathrm{O} 15-\mathrm{H} 12$ & $108(3)$ \\
\hline $\mathrm{O} 9{ }^{\mathrm{i}}-\mathrm{O} 15-\mathrm{H} 12$ & $103(3)$ \\
\hline $\mathrm{O} 4^{\mathrm{xi}}-\mathrm{O} 15-\mathrm{H} 12$ & $32(3)$ \\
\hline $\mathrm{O} 3^{\mathrm{x}}-\mathrm{O} 15-\mathrm{H} 12$ & $43(3)$ \\
\hline $\mathrm{O} 1^{\mathrm{x}}-\mathrm{O} 15-\mathrm{H} 12$ & $73(3)$ \\
\hline 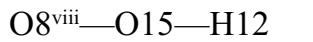 & $64(3)$ \\
\hline
\end{tabular}

$76.12(7)$

89.75 (13)

$69.95(8)$

$109.13(10)$

$50.47(5)$

$58.42(6)$

$106.35(7)$

$109.28(13)$

$123.31(10)$

$91.56(9)$

$112.11(7)$

$62.65(6)$

$128.92(7)$

$77.32(7)$

$83.39(12)$

$62.31(8)$

$105.04(9)$

$50.10(4)$

$99.67(7)$

$42.20(5)$

$90.17(7)$

$161.88(7)$

$29.33(11)$

$31.10(11)$

$60.43(8)$

$164.0(2)$

$137.71(14)$

$157.96(15)$

$95.90(15)$

$114.74(11)$

75.09 (9)

$84.07(10)$

$103.77(15)$

$75.16(9)$

$133.87(10)$

$62.61(9)$

$116.75(8)$

87.65 (14)

107.57 (9)

66.87 (8)

108.35 (11)

94.14 (8)

$145.13(10)$ 


\begin{tabular}{llll}
$\mathrm{N} 3-\mathrm{O} 8-\mathrm{O} 12$ & $131.37(16)$ & $\mathrm{O} 11-\mathrm{O} 15-\mathrm{H} 12$ & $162(3)$ \\
$\mathrm{O} 9-\mathrm{O} 8-\mathrm{O} 12$ & $160.61(11)$ & $\mathrm{H} 11-\mathrm{O} 15-\mathrm{H} 12$ & $105(5)$ \\
\hline
\end{tabular}

Symmetry codes: (i) $-x,-y+1,-z+1$; (ii) $x-1, y, z$; (iii) $-x,-y+1,-z$; (iv) $-x,-y+2,-z$; (v) $x, y-1, z$; (vi) $-x,-y,-z+1$; (vii) $-x+1,-y,-z+1$; (viii) $-x+1$, $-y+1,-z+1$; (ix) $-x+1,-y+1,-z$; (x) $x+1, y, z$; (xi) $x, y+1, z$.

Hydrogen-bond geometry $\left(A,{ }^{\circ}\right)$

\begin{tabular}{|c|c|c|c|c|}
\hline$D-\mathrm{H} \cdots A$ & $D-\mathrm{H}$ & $\mathrm{H} \cdots A$ & $D \cdots A$ & $D-\mathrm{H} \cdots A$ \\
\hline $\mathrm{O} 10-\mathrm{H} 1 \cdots \mathrm{O} 14$ & $0.85(4)$ & $1.89(4)$ & $2.730(3)$ & $170(4)$ \\
\hline $\mathrm{O} 10-\mathrm{H} 2 \cdots \mathrm{O} 15$ & $0.73(5)$ & $2.00(5)$ & $2.714(3)$ & $164(5)$ \\
\hline $\mathrm{O} 11-\mathrm{H} 4 \cdots \mathrm{O} 15^{\mathrm{viii}}$ & $0.74(4)$ & $1.93(4)$ & $2.666(2)$ & $174(4)$ \\
\hline $\mathrm{O} 11-\mathrm{H} 3 \cdots \mathrm{O}^{\mathrm{i}}$ & $0.81(4)$ & $2.20(4)$ & $3.006(2)$ & $175(4)$ \\
\hline $\mathrm{O} 12-\mathrm{H} 5 \cdots \mathrm{O} 8^{\mathrm{vii}}$ & $0.80(4)$ & $2.15(4)$ & $2.943(3)$ & $172(4)$ \\
\hline $\mathrm{O} 12-\mathrm{H} 6 \cdots 5^{\mathrm{x}}$ & $0.79(4)$ & $2.57(4)$ & $3.260(3)$ & $147(4)$ \\
\hline $\mathrm{O} 12-\mathrm{H} 6 \cdots \mathrm{O}^{\mathrm{x}}$ & $0.79(4)$ & $2.61(4)$ & $3.269(3)$ & $142(4)$ \\
\hline $\mathrm{O} 13-\mathrm{H} 7 \cdots \mathrm{O} 14^{\mathrm{ix}}$ & $0.78(4)$ & $1.93(4)$ & $2.713(3)$ & $174(4)$ \\
\hline $\mathrm{O} 13-\mathrm{H} 8 \cdots \mathrm{O}^{\mathrm{iii}}$ & $0.83(5)$ & $2.13(5)$ & $2.963(2)$ & $179(5)$ \\
\hline $\mathrm{O} 14-\mathrm{H} 9 \cdots \mathrm{O}^{\mathrm{iii}}$ & $0.80(4)$ & $2.01(4)$ & $2.804(3)$ & $176(4)$ \\
\hline $\mathrm{O} 14-\mathrm{H} 10 \cdots \mathrm{O}^{\mathrm{x}}$ & $0.80(5)$ & $2.64(5)$ & $3.111(3)$ & $119(4)$ \\
\hline $\mathrm{O} 14-\mathrm{H} 10 \cdots \mathrm{O}^{\mathrm{iv}}$ & $0.80(5)$ & $2.24(5)$ & $2.885(2)$ & $138(4)$ \\
\hline $\mathrm{O} 15-\mathrm{H} 11 \cdots \mathrm{O}^{\mathrm{i}}$ & $0.79(5)$ & $2.01(5)$ & $2.782(3)$ & $168(4)$ \\
\hline $\mathrm{O} 15-\mathrm{H} 12 \cdots \mathrm{O} 4^{\mathrm{xi}}$ & $0.79(5)$ & $2.29(5)$ & $2.926(2)$ & $137(4)$ \\
\hline $\mathrm{O} 15-\mathrm{H} 12 \cdots \mathrm{O}^{\mathrm{x}}$ & $0.79(5)$ & $2.47(5)$ & $2.992(3)$ & $125(4)$ \\
\hline
\end{tabular}

Symmetry codes: (i) $-x,-y+1,-z+1$; (iii) $-x,-y+1,-z$; (iv) $-x,-y+2,-z$; (vii) $-x+1,-y,-z+1$; (viii) $-x+1,-y+1,-z+1$; (ix) $-x+1,-y+1,-z$; (x) $x+1, y$, $z$; (xi) $x, y+1, z$. 